Decomposição open book generalizada em conjuntos semi-algébricos

Antonio Andrade do Espirito Santo 



\title{
Decomposição open book generalizada em conjuntos semi-algébricos
}

\author{
Antonio Andrade do Espirito Santo
}

Orientador: Prof. Dr. Raimundo Nonato Araújo dos Santos

Tese apresentada ao Instituto de Ciências Matemáticas e de Computação - ICMC-USP, como parte dos requisitos para obtenção do título de Doutor em Ciências - Matemática. VERSÃO REVISADA 
Ficha catalográfica elaborada pela Biblioteca Prof. Achille Bassi e Seção Técnica de Informática, ICMC/USP, com os dados fornecidos pelo(a) autor(a)

Espirito Santo, Antonio Andrade do Decomposição open book generalizada em conjuntos semi-algébricos / Antonio Andrade do Espirito Santo; orientador Raimundo Nonato Araújo dos Santos. -- São Carlos, 2014. $63 \mathrm{p}$.

Tese (Doutorado - Programa de Pós-Graduação em Matemática) -- Instituto de Ciências Matemáticas e de Computação, Universidade de São Paulo, 2014.

1. Decomposição open book generalizada. 2 . Estrutura de fibração em conjuntos semi-algébricos. 3. Topologia da singularidade. 4. Fibração de Milnor real e complexa. 5. Fibração local e global. I. Araújo dos Santos, Raimundo Nonato, orient. II. Título. 
"Não desisti pois tive ajuda"

Obrigado Deus 
"Adriana e Brenda. Hoje não sei o que significa viver, se eu não estiver com vocês. Agradeço a Deus que permitiu que estivéssemos juntos neste momento. Peço a Deus que nos permita que estejamos juntos em todos os momentos."

Dedico este trabalho a vocês: minhas vidas 


\section{Agradecimentos}

Ao completar este ciclo uma certeza não me falta, a de que sou muito grato ao Pai criador de todas as coisas, a quem me dirijo como Deus, que esteve comigo nos momentos em que a racionalidade já havia me abandonado, me consolando e me renovando as esperanças.

Agradeço aos membros da banca examinadora, que aceitaram avaliar este trabalho e apresentaram várias sugestões para melhorá-lo. Agradeço aos professores do Instituto de Ciências Matemáticas e de Computação (ICMC/USP), e aos queridos colegas de doutorado. Agradeço também a Universidade Federal do Recôncavo da Bahia (UFRB) e, em particular, a todos os professores do Centro de Ciências Exatas e Tecnológicas (CETEC/UFRB) e a Coordenação de Aperfeiçoamento de Pessoal de Nível Superior (CAPES), que através do Programa Prodoutoral, me concedeu apoio financeiro.

Gostaria de agradecer especialmente ao meu orientador, o Prof. Dr. Raimundo Santos, pela oportunidade de desenvolver este trabalho sob sua orientação, por não me abandonar nos momentos difíceis, por acreditar em mim e não me deixar desistir, pela ajuda, sem a qual a realização deste trabalho não seria possível. Também agradeço pela amizade, pelo exemplo, por sempre estar disponível para discutir sobre Matemática. O Prof. Raimundo além de ser um profissional dedicado, competente e extremamente responsável é também um ser humano generoso. Obrigado Professor.

Agradeço ao meu irmão de coração, Antonio Sande. Um servo fiel de Deus. Um incansável militante no recrutamento de pessoas para a obra de Deus. Um grande amigo que o bom e generoso Deus colocou em meu caminho.

Gostaria de agradecer ao meu Pai, Antonio Fidelis, pelos ensinamentos de toda a vida, pela confiança, por todos os exemplos de humildade, dignidade, bondade e honestidade. Meu exemplo para toda a minha vida.

Aos meus amores: Adriana e Brenda. Adriana, minha esposa-companheira e Brenda, minha filha, todos os meus agradecimentos. Vocês são os melhores presentes de Deus em minha vida, vocês são a razão da minha vida. Este momento só faz sentido para mim, pois vocês estão comigo.

Brenda, papai quer te dizer que o seu nascimento permitiu que eu pudesse chegar até aqui. De fato, você renovou a minha vontade de viver. Eu e sua mãe te amamos enormemente. Te amamos tanto que certamente não seria possível descrever esse amor com qualquer estrutura de linguagem conhecida.

Agradeço a minha Mãe, Valdelice. É difícil dizer que ela não está mais entre nós, pois 
sinto a presença dela a todo momento. E, principalmente, nos momentos em que mais preciso. Não é fácil explicar, e este também não é o meu propósito aqui, apenas gostaria de te dizer: obrigado Mãe. Te amo. 


\section{Resumo}

Nos últimos anos, vários pesquisadores tais como: A. Bodin, A. Dimca, A. Durfee, A. Jacquemard, A. Menegon Neto, A. Némethi, A. Pichon, A. Verjovsky, A. Zaharia, D. Siersma, H. A. Hamm, D. Massey, H. Aguilar-Cabrera, H. H. Vui, J. Cisneros, J. Seade, J. Snoussi, L. D. Tráng, L. Păunescu, L. R. Dias, M. A. S. Ruas, M. Oka, M. Tibăr, N. Dutertre, R. N. Araújo dos Santos, S. A. Broughton, T. Gaffney, Y. Chen, entre outros, têm apresentado generalizações dos Teoremas de fibrações de Milnor no ambiente real e complexo (e do Teorema de Kurdyka-Orro-Simon, ver por exemplo [Di, KOS]), visando um melhor entendimento de propriedades topológicas locais e globais das singularidades.

Nesta direção de pesquisa esses autores tem utilizado várias ferramentas e técnicas de diversas áreas da matemática. O que mostra a riqueza e a complexidade destes estudos e acrescenta, em nossa modesta opinião, um aspecto que é ao mesmo tempo interessante e desafiador.

Neste trabalho, mostraremos como estender as fibrações de Milnor em esferas no caso local e global, real e complexo, para uma aplicação $C^{2}$-semi-algébrica $F=\left(f_{1}, \ldots, f_{p}\right)$ : $\mathbb{R}^{N} \rightarrow \mathbb{R}^{p}$ e uma variedade $W \subset \mathbb{R}^{N}$ semi-algébrica com possível singularidade. Com tal objetivo, introduziremos as "condições de Milnor $(a)$ e $(b)$ generalizadas" e mostraremos como adaptar a técnica da decomposição open book superior com binding singular, introduzida em [AT, ACT1].

Nossos resultados sugerem que tal estrutura de fibração pode ser um caso particular de algum Teorema estrutural mais geral.

Além do mais, considerando $\pi: \mathbb{R}^{p} \rightarrow \mathbb{R}^{p-1}$ a projeção canônica na meta, mostraremos que se $F$ satisfaz tais condições, então $G=\pi \circ F: \mathbb{R}^{N} \rightarrow \mathbb{R}^{p-1}$ também satisfaz e, consequentemente, $G$ também induz em $W$ uma fibração suave localmente trivial.

Concluiremos mostrando que após as projeções as fibras destes fibrados são homotopicamente equivalentes e, em seguida, apresentando algumas fórmulas que relacionam a característica de Euler do "link relativo" $W \cap F^{-1}(0)$ com a característica de Euler das fibras.

Palavras-chave: decomposição open book generalizada, estrutura de fibração em conjuntos semi-algébricos, topologia da singularidade, fibração de Milnor real e complexa e fibração local e global. 


\section{Abstract}

In the last years, several researchers such as: A. Bodin, A. Dimca, A. Durfee, A. Jacquemard, A. Menegon Neto, A. Némethi, A. Pichon, A. Verjovsky, A. Zaharia, D. Siersma, H. A. Hamm, D. Massey, H. Aguilar-Cabrera, H. H. Vui, J. Cisneros, J. Seade, J. Snoussi, L. D. Tráng, L. Păunescu, L. R. Dias, M. A. S. Ruas, M. Oka, M. Tibăr, N. Dutertre, R. N. Araújo dos Santos, S. A. Broughton, T. Gaffney, Y. Chen, and others, have proven generalizations of Milnor fibrations's Theorems in the real and complex settings (and Kurdyka-Orro-Simon's Theorem, see e.g. [Di, KOS]), aiming a better understanding of the local and global topological properties of singularity.

In this research branch, these authors have used many different tools and techniques from several areas of Mathematics. This shows the richness and complexities of these studies and adds, in our modest opinion, an aspect that is simultaneously interesting and challenging.

In this work, we introduce the "generalized Milnor's conditions $(a)$ and $(b)$ " to show an extension of the Milnor fibration Theorems on spheres in the local and global cases, in the real and complex setting. For this, we consider a $C^{2}$-semi-algebraic mapping $F=$ $\left(f_{1}, \ldots, f_{p}\right): \mathbb{R}^{N} \rightarrow \mathbb{R}^{p}$, a possible singular semi-algebraic variety $W \subset \mathbb{R}^{N}$, and we show how to adapt the technique of Higher open book decomposition with singular binding, introduced by $[\mathrm{AT}, \mathrm{ACT} 1]$, to prove such extension.

Our results suggest that such fibration structure may be a particular case of a more general fibration structure.

Furthermore, considering $\pi: \mathbb{R}^{p} \rightarrow \mathbb{R}^{p-1}$ the canonical projection on the target space, we show that if $F$ satisfies the generalized Milnor's conditions $(a)$ and $(b)$, then $G=\pi \circ F$ : $\mathbb{R}^{N} \rightarrow \mathbb{R}^{p-1}$ also satisfies these conditions and, hence $G$ also induces on $W$ a smooth locally trivial fibration.

Finally, we show that after the projections on the target space, the fibers of these fiber bundles are homotopically equivalent. We conclude by proving some formulae connecting the Euler characteristic of "relative link" $W \cap F^{-1}(0)$ with the Euler characteristic of the fibers.

Key words and phrases: generalized open book decomposition, fibration structure on semi-algebraic sets, topology of singularity, real and complex Milnor's fibrations and, local and global fibration. 


\section{Sumário}

$\begin{array}{ll}\text { Introdução } & 1\end{array}$

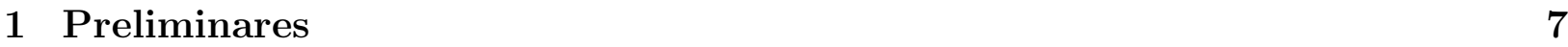

1.1 Conjuntos semi-algébricos . . . . . . . . . . . . . . . . 7

1.2 Variedades com bordo . . . . . . . . . . . . . . . . . . . . 10

1.3 Teoria de Morse para variedades com bordo . . . . . . . . . . . . . . 11

1.3.1 Variedades com corners . . . . . . . . . . . . . . . 15

1.3.2 Teorema de fibração para variedades com bordo . . . . . . . . . . . 17

2 Classes Especiais de Polinômios $\quad 19$

2.1 Polinômios Newton não-degenerados no infinito . . . . . . . . . . . . . . . . 19

2.2 Polinômios Tame . . . . . . . . . . . . . . . . . . . . . . . . . . 22

2.3 Polinômios Quasitame e $M$-Tame . . . . . . . . . . . . . . . . . . . . . . . . 24

2.4 Polinômios Semitame . . . . . . . . . . . . . . . . . . . . . . . . 28

3 Fibrações em Esferas $\quad 31$

3.1 O Teorema de fibração para polinômios Semitame . . . . . . . . . . . . . . 31

3.2 Decomposição open book . . . . . . . . . . . . . . . . . . . . . . 37

4 Um Teorema Estrutural para Conjuntos Semi-Algébricos 41

4.1 As condições de Milnor $(a)$ e $(b)$ generalizadas . . . . . . . . . . . . . . . 41

4.2 Estrutura open book de aplicações polinomiais . . . . . . . . . . . . . . . 43

4.3 Uma condição semi-algébrica . . . . . . . . . . . . . . . . . . . . 44 
5 Topologia da Fibra de Milnor no Infinito

5.1 Relação entre as fibras $M_{F}$ e $M_{G} \ldots \ldots \ldots \ldots$. . . . . . . . . . 52

5.2 A característica de Euler das fibras e dos links relativos . . . . . . . . . . 57

Referências Bibliográficas

60 


\section{Introdução}

Dada $f:\left(\mathbb{C}^{m+1}, 0\right) \rightarrow(\mathbb{C}, 0)$ germe de função holomorfa, John Milnor, em 1968, no livro Singular Points of Complex Hypersurfaces [Mil1], estudou a topologia da hipersuperfície complexa $V=f^{-1}(0):=\left\{z \in \mathbb{C}^{m+1}: f(z)=0\right\}$ na vizinhança da origem $0 \in \mathbb{C}^{m+1}$, intersectando-a com uma pequena esfera, $S_{\epsilon}^{2 m+1}:=\left\{z \in \mathbb{C}^{m+1}:\|z\|=\epsilon\right\}$. Milnor mostrou que sendo $f: \mathcal{U} \subset \mathbb{C}^{m+1} \rightarrow \mathbb{C}$ um representante de um germe de uma função holomorfa, existe um número real $\epsilon_{0}>0$ suficientemente pequeno, tal que para todo $0<\epsilon \leq \epsilon_{0}$, a aplicação

$$
\phi:=\frac{f}{\|f\|}: S_{\epsilon}^{2 m+1} \backslash K_{\epsilon} \rightarrow S^{1}
$$

é a projeção de um fibrado suave localmente trivial, em que $K_{\epsilon}:=V \cap S_{\epsilon}^{2 m+1}$ é chamado o link da singularidade na origem.

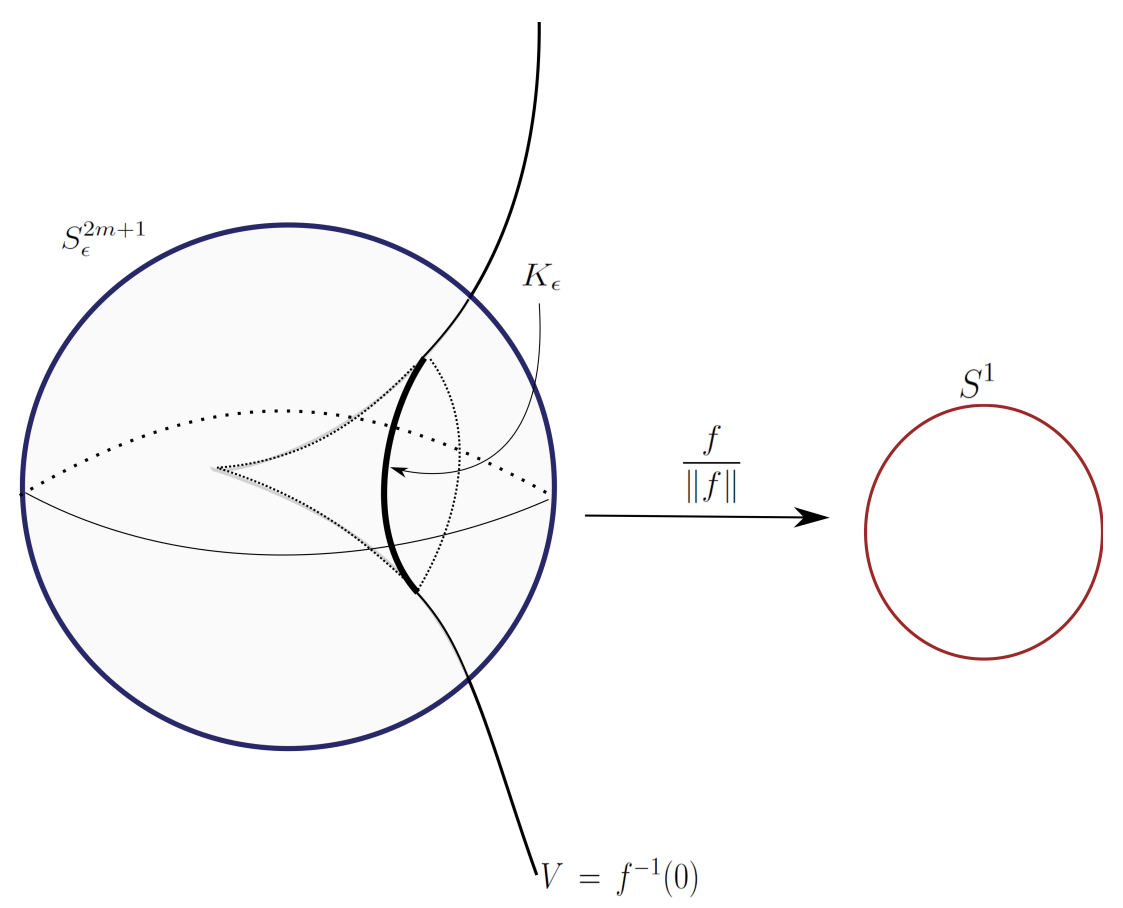

Milnor usou ferramentas da Teoria de Morse e descreveu algumas propriedades da topologia da fibra como segue:

(a) A fibra é paralelizável, ou seja, seu fibrado tangente é trivial. 
(b) A fibra tem o mesmo tipo de homotopia de um $C W$-complexo finito de dimensão $m$;

(c) O link $K_{\epsilon}$ é $(m-2)$-conexo, isto é, $\pi_{i}\left(K_{\epsilon}\right)=0$, para todo $0 \leq i \leq m-2$. Assim, para $m=2$ o link é conexo e para $m \geq 3$ o link é simplesmente conexo.

Ademais, sendo a origem um ponto singular isolado da $f$, Milnor refinou os resultados da seguinte maneira:

(d) O link $K_{\epsilon}$ é uma variedade suave $(2 m-1)$-dimensional mergulhada em $S_{\epsilon}^{2 m+1}$;

(e) $\mathrm{O}$ fecho topológico da fibra $F_{\theta}:=\phi^{-1}(\theta), \overline{F_{\theta}}$, é uma variedade compacta com bordo $K_{\epsilon}$ e está mergulhada em $S_{\epsilon}^{2 m+1}$ de tal modo que tem o mesmo tipo de homotopia do seu complementar $S_{\epsilon}^{2 m+1} \backslash \overline{F_{\theta}}$;

(f) A fibra é $(m-1)$-conexa e tem o tipo de homotopia de um bouquet de esferas $m$ dimensionais $S^{m} \vee \cdots \vee S^{m}$ e o número de esferas neste bouquet é dado pelo grau topológico da aplicação

$$
\epsilon \frac{\nabla f}{\|\nabla f\|}: S_{\epsilon}^{2 m+1} \rightarrow S_{\epsilon}^{2 m+1}
$$

denotado por $\mu:=\operatorname{deg}_{0}(\nabla f)$.

Este número foi chamado posteriormente de número de Milnor. Daí, podemos concluir que a característica de Euler-Poincaré da fibra $F_{\theta}$ é

$$
\chi\left(F_{\theta}\right)=1+(-1)^{m} \mu .
$$

Para o caso de germe de aplicações analíticas reais $F=\left(f_{1}, \cdots, f_{p}\right):\left(\mathbb{R}^{n}, 0\right) \rightarrow\left(\mathbb{R}^{p}, 0\right)$ com ponto crítico isolado na origem, Milnor provou que a aplicação

$$
F_{\mid}: B_{\epsilon}^{n} \cap F^{-1}\left(S_{\eta}^{p-1}\right) \rightarrow S_{\eta}^{p-1}
$$

é uma fibração suave localmente trivial, em que $B_{\epsilon}^{n}$ é a bola fechada do $\mathbb{R}^{n}$ com centro na origem e raio $\epsilon>0$. Tal fibração foi chamada posteriormente de fibração no tubo de Milnor.

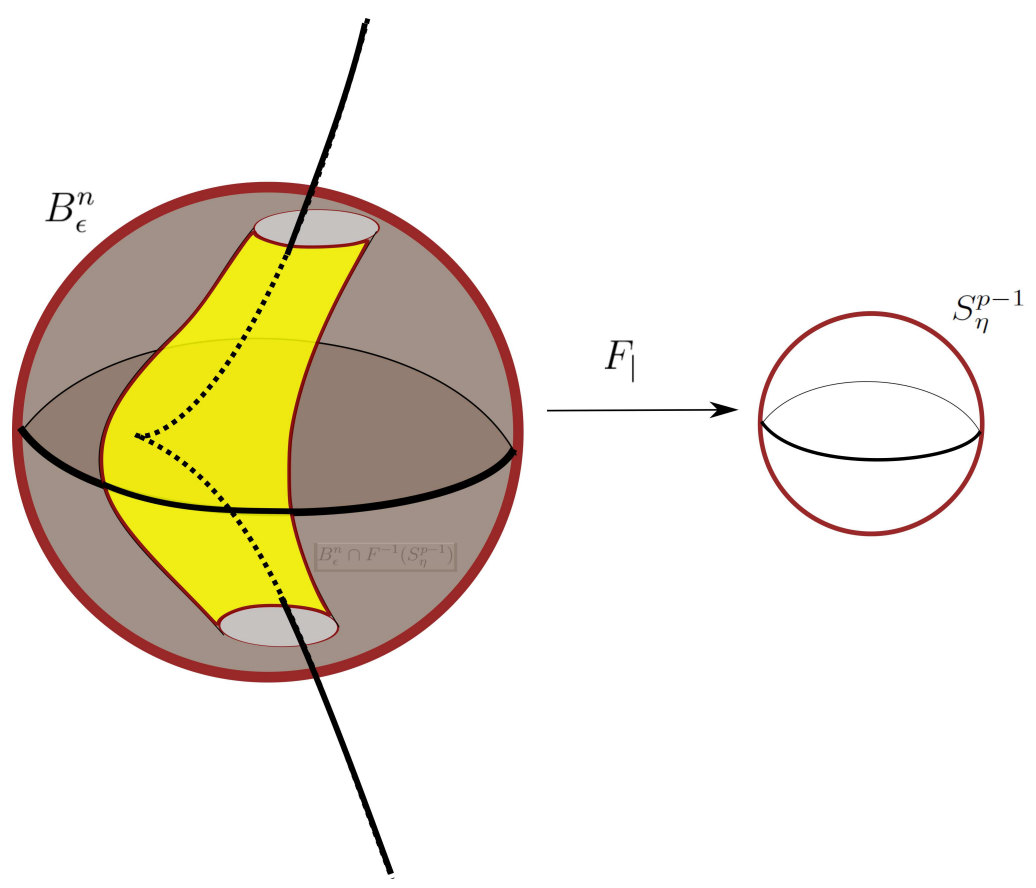


Em seguida, usando o fluxo de um campo de vetores conveniente, Milnor mostrou como inflar o tubo $B_{\epsilon}^{n} \cap F^{-1}\left(S_{\eta}^{p-1}\right)$ para a esfera $S_{\epsilon}^{n-1}$, de modo a garantir que a aplicação

$$
\varphi: S_{\epsilon}^{n-1} \backslash K_{\epsilon} \rightarrow S_{\eta}^{p-1}
$$

fosse também uma fibração suave localmente trivial. No entanto, como foi observado em [Mil1, capítulo 11, pg. 99] a projeção deste último fibrado não é dada, em geral, pela aplicação $\frac{F}{\|F\|}$ como acontece no caso de função holomorfa. Este problema foi abordado posteriormente por vários matemáticos, por exemplo, em [Ar, Ja, RSV] e caracterizado pelos autores em [AT, JJJ].

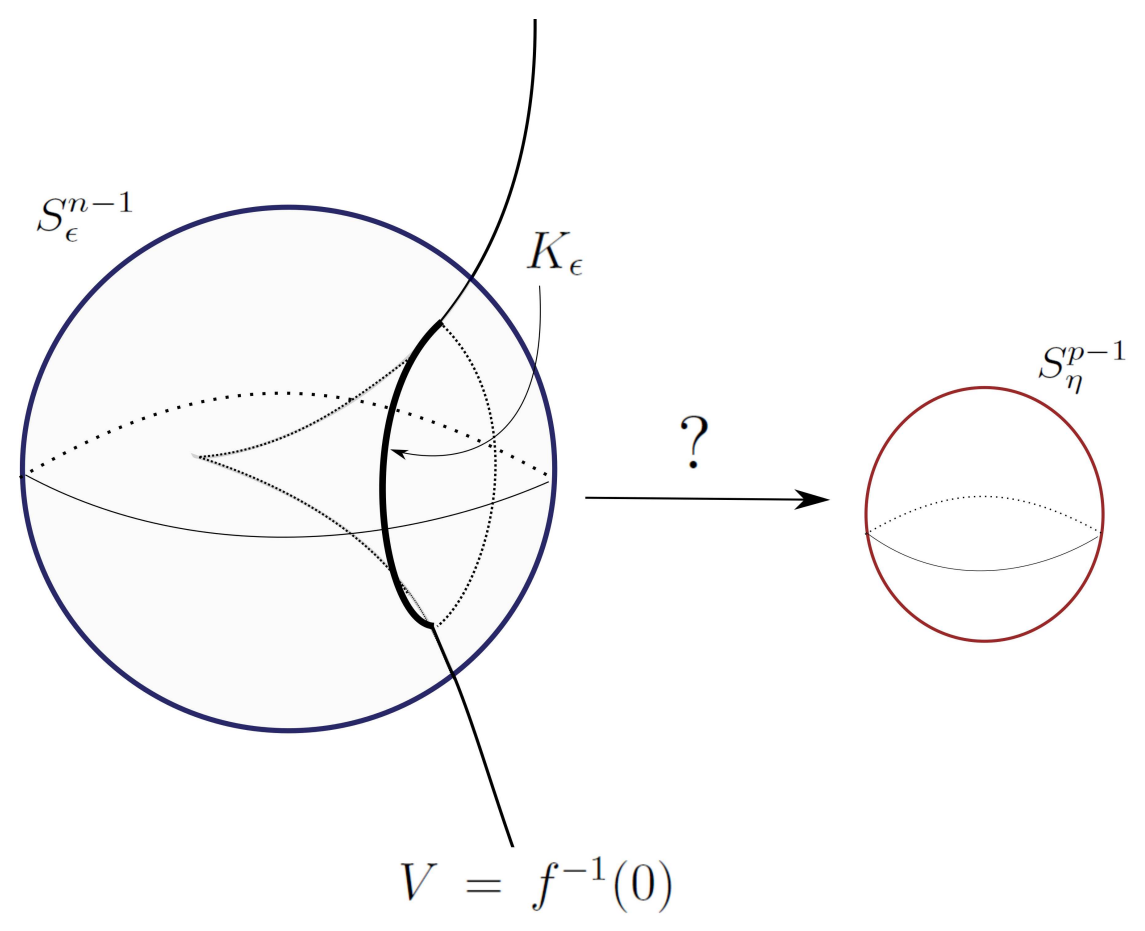

Milnor também mostrou que se o link for não vazio ele é uma variedade suave de dimensão $n-p-1$ mergulhada na esfera com fibrado normal trivial e que a fibra da fibração (5) é pelo menos $(p-2)$ conexa.

Mais recentemente, no artigo Topology of real Milnor fiber for isolated singularities [ADD] utilizando ferramentas da Teoria de Morse para variedades com bordo, R. Araújo dos Santos, D. Dreibeibris e N. Dutertre demonstraram uma extensão da fórmula de Khinshiasvilli [Kh] e da equação (3), como abaixo. Denotaremos a seguir por $M_{F}$ a fibra da fibração (4).

(a) Se $n$ é par, então $\chi\left(M_{F}\right)=1-\operatorname{deg}_{0} \nabla f_{1}$. Além disso,

$$
\operatorname{deg}_{0} \nabla f_{1}=\operatorname{deg}_{0} \nabla f_{2}=\cdots=\operatorname{deg}_{0} \nabla f_{p} .
$$

(b) Se $n$ é ímpar, então $\chi\left(M_{F}\right)=1$. Além disso, $\operatorname{deg}_{0} \nabla f_{i}=0, i=1,2, \ldots, p$.

Nos últimos anos, esses resultados foram estendidos para singularidades reais não-isoladas. Por exemplo, em [Ma2] David Massey considerou as chamadas condições de Milnor (a) e 
(b) e provou que tais condições são suficientes para garantir a existência da fibração no tubo de Milnor (4). Em [AT, ACT] tais condições foram adaptadas pelos autores para mostrar a existência da fibração (5) com projeção dada por $\frac{F}{\|F\|}$.

Observamos que no caso holomorfo as condições introduzidas por D. Massey em [Ma2] são automaticamente satisfeitas: de fato, a condição $(a)$ assegura que localmente o conjunto singular está contido no conjunto dos zeros, o que é verdadeiro no caso holomorfo devido a desigualdade de Łojasiewicz. A condição (b) assegura que as fibras próximas do conjunto dos zeros intersectam as pequenas esferas transversalmente, o que é verdadeiro no caso holomorfo devido a condição $a_{F}$ de Thom.

No caso global, é conhecido que para funções polinomiais holomorfas $f: \mathbb{C}^{n+1} \rightarrow \mathbb{C}$ existe um conjunto finito de valores complexos chamado conjunto de bifurcação, denotado por $B_{f}$, tal que a aplicação restrição

$$
f_{\mid}: \mathbb{C}^{n+1} \backslash f^{-1}\left(B_{f}\right) \rightarrow \mathbb{C} \backslash B_{f}
$$

é uma fibração suave localmente trivial. ${ }^{1}$ Logo, considerando um disco fechado $D_{R} \subset \mathbb{C}$, com $R \gg 1$, isto é, $R>0$ suficientemente grande, de tal forma que $B_{f}$ está contido em seu interior, temos que esta fibração induz a chamada monodromia global de $f$

$$
f_{\mid}: \mathbb{C}^{n+1} \cap f^{-1}\left(S_{R}^{1}\right) \rightarrow S_{R}^{1}
$$

a qual é também um fibração suave localmente trivial.

Em [NZ1] Némethi e Zaharia consideraram uma condição de regularidade chamada semitame, que controla o comportamento assintótico das fibras no infinito. Seguindo o método de Milnor para o caso local, eles provaram que para todo $R \gg 1$, a projeção de Milnor canônica

$$
\frac{f}{\|f\|}: S_{R}^{2 n+1} \backslash\{f=0\} \rightarrow S^{1}
$$

é uma fibração suave localmente trivial. No entanto, o exemplo do Broughton $f(x, y)=$ $x+x^{2} y$ mostra que a fibra da projeção de Milnor (8) é uma 2-esfera menos três pontos, enquanto que a fibra genérica (7) é uma 2-esfera menos dois pontos. Assim, de forma geral, estas duas fibrações podem não ser fibra-equivalentes.

Denotando por $\nabla f(z):=\left(\frac{\partial f}{\partial z_{0}}(z), \cdots, \frac{\partial f}{\partial z_{n}}(z)\right)$ o campo gradiente de uma função holomorfa $f$ e por $\bar{\nabla} f(z)$ seu conjugado complexo, em [NZ1] foi definido o conjunto de Milnor de $f$ dado por $M(f):=\left\{z \in \mathbb{C}^{n+1}: \bar{\nabla} f(z)=\lambda z\right.$, para algum $\left.\lambda \in \mathbb{C}\right\}$ e foi considerado o seguinte comportamento assintótico no infinito:

$(*)$ : para toda sequência $\left\{z_{k}\right\} \subset M(f)$ tal que $\left\|z_{k}\right\| \rightarrow+\infty$ e $\bar{\nabla} f\left(z_{k}\right) \rightarrow 0$.

Com isto, dada uma função polinomial holomorfa $f$ satisfazendo $(*)$, foi definido que:

(a) $f$ é $M$-tame, se $\left|f\left(z_{k}\right)\right| \rightarrow+\infty$.

\footnotetext{
${ }^{1}$ ver por exemplo [Br2, Proposição 1, pg. 168]
} 
(b) $f$ é semitame, se $f\left(z_{k}\right) \rightarrow c$, para algum $c \in \mathbb{C}$, então $c=0$.

É claro que, se $f$ é $M$-tame, então ela é semitame. Mas, a recíproca não é verdade, em geral, como pode ser visto em [NZ1, observação 7, pg. 330]. No entanto, sendo $f M$-tame as seguintes afirmações são verdadeiras:

(c) O conjunto singular, $\Sigma_{f}$, é formado apenas de pontos isolados. Logo, existe $R_{0} \gg 1$ tal que $\forall R \geq R_{0}, \Sigma_{f} \subset \stackrel{\circ}{B_{R}}$, em que $\dot{B}_{R}$ denota o interior da bola fechada $B_{R}^{2 n+2}$. Portanto, a aplicação

$$
R \frac{\nabla f}{\|\nabla f\|}: S_{R}^{2 n+1} \rightarrow S_{R}^{2 n+1}
$$

está bem definida e denotaremos seu grau topológico por $\operatorname{deg}_{\infty}(\nabla f)$.

(d) O conjunto de bifurcação $B_{f}$ é igual a $f\left(\Sigma_{f}\right)$;

(e) Para cada valor regular $w \in \mathbb{C} \backslash B_{f}$, a fibra (típica) $f^{-1}(w)$ tem o tipo de homotopia de um bouquet de esferas $n$-dimensionais. Neste caso, o número de esferas deste bouquet é também denotado por $\mu_{f}$, e pode ser provado que $\mu_{f}=\operatorname{deg} g_{\infty}(\nabla f)$;

(f) As fibrações (7) e (8) são fibra-equivalentes;

(g) $K_{R}$ é $(n-3)$-conexo, em que $K_{R}:=f^{-1}(0) \cap S_{R}^{2 n+1}$ é chamado o link no infinito de $f^{-1}(0) .^{2}$

Segue do item (e) acima que na classe de funções polinomiais $M$-tame ainda é válida a seguinte fórmula

$$
\chi\left(F_{\theta}\right)=1+(-1)^{n} \mu_{f} .
$$

No entanto, observamos que no caso semitame tal fórmula não faz sentido, visto que $\Sigma_{f} \subset$ $\{f=0\}$ pode ser não isolado (ver exemplo 2.2 - página 21 ).

No caso global real, Kurdyka, Orro e Simon [KOS, Teorema 3.1, pg. 78] consideraram aplicações reais $F: \mathbb{R}^{n} \rightarrow \mathbb{R}^{p} C^{2}$-semi-algébricas e mostraram que existe um subconjunto semi-algébrico fechado $B_{F}$ com $\operatorname{dim}_{\mathbb{R}} B_{F} \leq p-1$ tal que

$$
F_{\mid}: \mathbb{R}^{n} \backslash F^{-1}\left(B_{F}\right) \rightarrow \mathbb{R}^{p} \backslash B_{F}
$$

é uma fibração suave localmente trivial de classe $C^{2}$ em cada componente conexa de $\mathbb{R}^{p} \backslash B_{F}$.

O resultado anterior nos diz que o tipo topológico da fibra pode depender da componente conexa de $\mathbb{R}^{p} \backslash B_{F}$.

Um problema interessante nessa direção de pesquisa é encontrar ferramentas e técnicas que possam descrever o comportamento topológico das fibras quando mudamos de componente conexa através do conjunto de bifurcação $B_{F}$.

Este trabalho está organizado da seguinte forma.

\footnotetext{
${ }^{2}$ Devido a condição $a_{f}$ de Thom no infinito é bem conhecido que o tipo topológico de $K_{R}$ não muda para todo $R \gg 1$.
} 
No capítulo 1 apresentaremos algumas definições e resultados básicos sobre os conjuntos semi-algébricos, sobre variedades com bordo e Teoria de Morse para essas variedades. Tais variedades aparecem naturalmente nos principais resultados obtidos na Tese. Finalizaremos com a definição de fibração que utilizaremos ao longo do trabalho e com o Teorema de fibração de Ehresmann para variedades com bordo.

No capítulo 2 apresentaremos algumas classes especiais de polinômios complexos; a saber, tame, quasitame, $M$-tame e semitame, que motivaram o desenvolvimento de várias condições assintóticas, as quais permitem controlar o comportamento da fibra de uma função polinomial no infinito.

No capítulo 3 apresentaremos resultados que garantem a existência da fibração (8) em grandes esferas para as classes de polinômios complexos apresentados no capítulo 2. Além disso, apresentaremos uma técnica chamada decomposição "open book no infinito" que generaliza a fibração (8) para aplicações polinomiais reais.

Nos capítulos 4 e 5 apresentaremos as principais contribuições obtidas na Tese e seguire$\operatorname{mos}[\mathrm{DAYA}]$.

No capítulo 4 considerando $W^{n} \subset \mathbb{R}^{N}$ uma variedade suave compacta sem bordo semialgébrica de dimensão $n, F=\left(f_{1}, \ldots, f_{p}\right): \mathbb{R}^{N} \rightarrow \mathbb{R}^{p}, 2 \leq p \leq n<N$, uma aplicação $C^{2}$-semi-algébrica e $\bar{F}:=\frac{F}{\|F\|}: \mathbb{R}^{N} \backslash V(F) \rightarrow S^{p-1}$, em que $V(\bar{F}):=F^{-1}(0)$, provaremos que sob as chamadas condições de Milnor $(a)$ e $(b)$ generalizadas a aplicação restrição $\bar{F}$ : $W \backslash V_{W}(F) \rightarrow S^{p-1}$, em que $V_{W}(F):=V(F) \cap W$, é uma fibração suave localmente trivial (Teorema 4.1 - página 42). Mostraremos também que tais condições estendem a técnica de decomposição open book apresentada no capítulo anterior para o caso global complexo e real. Além disso, mostraremos que nosso resultado também estende os Teoremas de fibração de Milnor local no caso real e complexo. Em seguida, considerando a aplicação composição $G:=\pi \circ F:\left(f_{1}, \ldots, f_{p-1}\right): \mathbb{R}^{N} \rightarrow \mathbb{R}^{p-1}, p \geq 3$, em que $\pi$ é a projeção canônica na meta, mostraremos que $G$ também satisfaz as condições de Milnor $(a)$ e $(b)$ generalizadas, e consequentemente, $\bar{G}: W \backslash V_{W}(G) \rightarrow S^{p-2}$ também define uma fibração suave localmente trivial (Teorema 4.10 - página 47 ).

No capítulo 5 consideraremos as fibrações $\bar{F}: W \backslash V_{W}(F) \rightarrow S^{p-1}$ e $\bar{G}: W \backslash V_{W}(G) \rightarrow$ $S^{p-2}, p \geq 3$, e mostraremos que suas respectivas fibras $M_{F}$ e $M_{G}$ são homotopicamente equivalentes (Teorema 5.3 - página 52). Portanto, o estudo da topologia das fibras destes fibrados pode ser reduzido para o caso $p=2$. Neste caso, mostraremos que o estudo da topologia da fibra depende apenas do estudo da topologia do link das funções coordenadas (Teorema 5.10 - página 57). Em seguida mostraremos como relacionar a característica de Euler do "link relativo", $V_{W}(F)$, com a característica de Euler da fibra $M_{F}$ (Teorema 5.17 página 59).

Ressaltamos que, nesta direção de estudos, ainda se faz necessário um desenvolvimento de técnicas e ferramentas que possibilitem obter informações mais precisas sobre a topologia das fibras e dos links destas fibrações. 


\section{Capítulo 1}

\section{Preliminares}

\section{Sumário}

1.1 Conjuntos semi-algébricos ............... 7

1.2 Variedades com bordo .................. 10

1.3 Teoria de Morse para variedades com bordo ......... 11

1.3.1 Variedades com corners . . . . . . . . . . . . 15

1.3.2 Teorema de fibração para variedades com bordo . . . . . . . . . . 17

Ao longo deste trabalho, usaremos as seguintes convenções: as palavras "suave" e "diferenciáveis" serão usadas indistintamente para significar diferenciável de classe $C^{\infty} ; M^{m}$ para significar uma variedade suave $m$-dimensional, mergulhada em $\mathbb{R}^{N}$, para algum $N$ (isto é possível devido ao Teorema do mergulho de Whitney). Quando não houver perigo de confusão, simplesmente denotaremos por $M$ uma tal variedade. O espaço tangente a variedade suave $M$ em um ponto $x$ será denotado por $T_{x} M$. Por fim, se $F: M \rightarrow N$ é uma aplicação suave com $F(x)=y$, então a aplicação linear induzida entre os espaços tangentes de $M$ e $N$, respectivamente, será denotada por $D F(x): T_{x} M \rightarrow T_{y} N$. Além disso, usaremos o símbolo $\sim$ quando duas variedades $M$ e $N$ forem homeomorfas (respectivamente difeomorfas), e denotaremos por $X \simeq Y$ quando um espaço topológico $X$ for homotópico a $Y$.

Neste capítulo, iniciaremos com uma breve introdução sobre conjuntos algébricos e semialgébricos, variedades com bordo e Teoria de Morse sobre tais variedades. Em seguida, enunciaremos o Teorema de fibração de Ehresmann para variedade com bordo.

Nossas principais referências são [Er, BCR, BR, HL, Mil2].

\subsection{Conjuntos semi-algébricos}

A seguir, vamos lembrar algumas definições e propriedades de aplicações e conjuntos algébricos. 
Definição 1.1 Um subconjunto $A \subset \mathbb{R}^{n}$ é chamado um conjunto algébrico, se $A$ é localmente o conjunto de zeros comuns de alguma coleção finita de funções polinomiais em $\mathbb{R}^{n}$.

Proposição $1.1 \quad$ (a) Sejam $A$ e $B$ conjuntos algébricos em $\mathbb{R}^{n}$. Então, $A \cup B$ e $A \cap B$ são conjuntos algébricos;

(b) Sejam $C \subset \mathbb{R}^{p}$ conjunto algébrico e $F: \mathbb{R}^{n} \rightarrow \mathbb{R}^{p}$ uma aplicação polinomial. Então $F^{-1}(C) \subset \mathbb{R}^{n}$ é um conjunto algébrico.

Os conjuntos algébricos gozam de "boas" propriedades, conforme mostra a Proposição 1.1 acima. No entanto, o próximo exemplo mostra que a imagem direta de um conjunto algébrico por uma aplicação polinomial nem sempre é um conjunto algébrico.

Exemplo 1.2 Sejam $\pi: \mathbb{R}^{2} \rightarrow \mathbb{R}$ a projeção na primeira coordenada e $S^{1}:=\left\{(x, y) \in \mathbb{R}^{2}\right.$ : $\left.x^{2}+y^{2}=1\right\}$ a esfera unitária. Temos que $S^{1}$ é um conjunto algébrico, mas $\pi\left(S^{1}\right)=[-1,1]$ não o é. De fato, para todo polinômio $f: \mathbb{R} \rightarrow \mathbb{R}$, temos duas possibilidades para $f^{-1}(0)$. Se $f$ não for identicamente nulo, temos que $f^{-1}(0)$ é um conjunto finito de raízes do polinômio. Caso contrário $f^{-1}(0)$ é toda a reta, logo não existe um polinômio tal que $f^{-1}(0)=[-1,1]$.

Portanto, é natural considerar a menor família de subconjuntos de $\mathbb{R}^{n}$ que contém todos os conjuntos algébricos e é fechada sobre projeções. Tal classe de conjuntos é chamada de semi-algébricos, que definiremos a seguir.

Definição 1.2 Um subconjunto $V \subset \mathbb{R}^{n}$ é chamado semi-algébrico, se ele admite uma representação da forma:

$$
V=\bigcup_{i=1}^{s} \bigcap_{j=1}^{r_{j}}\left\{x \in \mathbb{R}^{n}: P_{i j}(x) \sigma_{i, j} 0\right\},
$$

em que, $\sigma_{i, j} \in\{<,=,>\}$ e $P_{i j} \in \mathbb{R}\left[x_{1}, \cdots, x_{n}\right]$ para cada $i=1, \cdots$, s e $j=1, \cdots, r_{j}$.

\section{Exemplo 1.3}

(a) Os conjuntos algébricos, são claramente, conjuntos semi-algébricos;

(b) Reuniões finitas de pontos e intervalos abertos da reta $\mathbb{R}$ são conjuntos semi-algébricos. De fato, estes são os únicos subconjuntos semi-algébricos não-vazios de $\mathbb{R}$;

(c) As bolas abertas, fechadas, as esferas e os poliedros nos espaços euclidianos são conjuntos semi-algébricos de $\mathbb{R}^{n}$;

(d) Dados $A \subset \mathbb{R}^{p}$ um subconjunto semi-algébrico e $F: \mathbb{R}^{n} \rightarrow \mathbb{R}^{p}$ uma aplicação polinomial, temos que a pré-imagem $F^{-1}(A) \subset \mathbb{R}^{n}$ é um conjunto semi-algébrico.

Os resultados a seguir fornecem informações básicas importantes sobre a estrutura topológica dos conjuntos semi-algébricos. 
Proposição 1.4 Se $A \subset \mathbb{R}^{n}$ é um conjunto semi-algébrico, então o fecho de $A$ é um conjunto semi-algébrico.

Proposição 1.5 Se $A \subset \mathbb{R}^{n}$ é um conjunto semi-algébrico, então A tem um número finito de componentes conexas e cada uma das componentes é ainda um conjunto semi-algébrico.

Definição 1.3 Sejam $A \subset \mathbb{R}^{n}$ e $B \subset \mathbb{R}^{m}$ conjuntos semi-algébricos. Uma aplicação $F$ : $A \rightarrow B$ é chamada semi-algébrica, se seu gráfico é um conjunto semi-algébrico de $\mathbb{R}^{n+m}$.

Corolário 1.6 Sejam $A \subset \mathbb{R}^{n}, B \subset \mathbb{R}^{m}$ e $C \subset \mathbb{R}^{p}$ conjuntos semi-algébricos e uma aplicação $F=\left(f_{1}, f_{2}\right): A \rightarrow B \times C$. Então, $F$ é semi-algébrica se, e somente se, $f_{1}$ e $f_{2}$ são aplicações semi-algébricas. Além disso, a soma, o produto e a composição de aplicações semi-algébricas é semi-algébrica.

A seguir enunciamos o famoso Teorema de Tarski-Seidenberg, o qual estabelece que a projeção de conjunto semi-algébrico é semi-algébrico.

Teorema 1.7 (Teorema de Tarski-Seidenberg) A imagem de um conjunto semi-algébrico $A \subset \mathbb{R}^{n}$ por uma projeção $\pi: \mathbb{R}^{n} \rightarrow \mathbb{R}^{n-1}$ é um conjunto semi-algébrico.

Proposição 1.8 Sejam $A \subset \mathbb{R}^{n}, B \subset \mathbb{R}^{m}$ conjuntos semi-algébricos e $F: A \rightarrow B$ uma aplicação semi-algébrica. Então, a imagem $F(A) \subset B$ é um conjunto semi-algébrico.

Corolário 1.9 A imagem direta de um conjunto semi-algébrico por uma aplicação polinomial é um conjunto semi-algébrico.

Lema 1.10 (Lema de seleção da curva local) [BR, Proposição 2.6.19] Seja $A \subset \mathbb{R}^{n}$ um conjunto semi-algébrico. Seja $x \in \bar{A}$ e $x \notin A$, em que $\bar{A}$ denota o fecho topológico de $A$. Então, existe uma aplicação contínua semi-algébrica $\gamma:[0,1] \rightarrow \mathbb{R}^{n}$ tal que

(a) $\gamma(0)=x$;

(b) $\gamma:(0,1] \rightarrow \mathbb{R}^{n}$ é analítica;

(c) $\gamma((0,1]) \subseteq A$.

Na página 16 veremos uma aplicação interessante do Lema anterior.

Lema 1.11 (Lema de seleção da curva global) [NZ1, Lema 2, pg. 325] Sejam $f_{1}, \cdots, f_{q}$, $g_{1}, \cdots, g_{s}, h_{1}, \cdots, h_{r}$ em $\mathbb{R}\left[x_{1}, \cdots, x_{n}\right]$ funções polinomiais com coeficientes reais, e $\mathcal{U}=$ $\left\{x \in \mathbb{R}^{n}: f_{i}(x)=0, i=1, \cdots, q\right\}$ e $W=\left\{x \in \mathbb{R}^{n}: g_{i}(x)>0, i=1, \cdots, s\right\} . S u-$ ponha que existe uma sequência $\left\{x_{k}\right\} \subseteq \mathcal{U} \cap W$ tal que $\lim _{k \rightarrow \infty}\left\|x_{k}\right\|=\infty$ e para todo $j \in$ $\{1, \cdots, r\}, \lim _{k \rightarrow \infty} h_{j}\left(x_{k}\right)=0$. Então existe uma curva analítica real $p:(0, \epsilon) \rightarrow \mathcal{U} \cap W$, $p(t)=a_{0} t^{\alpha}+a_{1} t^{\alpha+1}+\cdots, \alpha<0$ e $a_{0} \in \mathbb{R}^{n} \backslash\{0\}$, tal que $\lim _{t \rightarrow 0}\|p(t)\|=\infty e \lim _{t \rightarrow 0} h_{j}(p(t))=0$ para $1 \leq j \leq r$. 
Aplicação 1.12 Sejam $g: \mathbb{R}^{n} \rightarrow \mathbb{R} \in C^{1}$ semi-algébrica, $X=g^{-1}(0)$ um conjunto ilimitado, $\Sigma(X)$ o conjunto singular de $X$ e $\rho: \mathbb{R}^{n} \rightarrow \mathbb{R}, \rho(x):=\|x\|^{2}$. Então, $\rho: X \backslash \Sigma(X) \rightarrow \mathbb{R}$ tem uma quantidade finita de valores críticos.

DemonstraÇÃo: De fato, suponha que exista uma sequência $\left\{y_{k}\right\} \subset \mathbb{R}$ de valores críticos de $\rho_{\mid}$com $\left\|y_{k}\right\| \rightarrow \infty$ quando $k \rightarrow \infty$. Então, existe uma sequência $\left\{x_{k}\right\} \subset X \backslash \Sigma(X)$ com $\left\|x_{k}\right\| \rightarrow \infty$, tal que $\rho\left(x_{k}\right)=\left\|x_{k}\right\|^{2}=y_{k}$. Portanto, pelo Lema anterior existe uma curva analítica $\varphi(t) \subset X \backslash \Sigma(X)$ tal que $\|\varphi(t)\| \rightarrow \infty$ quando $t \rightarrow 0$, com $\varphi(t) \subset \Sigma_{\rho_{\mid}}$, em que $\Sigma_{\rho_{\mid}}$ denota o conjunto singular de $\rho_{\mid}$. Logo, existe $\lambda(t) \neq 0$ tal que $\nabla g(t)=\lambda(t) \nabla \rho(t)$. Então,

$$
\begin{aligned}
0=\frac{d}{d t}(g(\varphi(t)))=\left\langle\nabla g(t), \varphi^{\prime}(t)\right\rangle & =2 \lambda(t)\left\langle\varphi(t), \varphi^{\prime}(t)\right\rangle \\
& =\lambda(t) \frac{d}{d t}\left(\|\varphi(t)\|^{2}\right)
\end{aligned}
$$

Como $\lambda(t) \neq 0$ segue que $\|\varphi(t)\|^{2}=$ cte, o que é um absurdo.

\subsection{Variedades com bordo}

Consideremos o semi-espaço fechado do $\mathbb{R}^{n}$, dado por $\mathbb{H}^{n}:=\left\{\left(x_{1}, x_{2}, \ldots, x_{n}\right) \in \mathbb{R}^{n}: x_{n} \geqslant\right.$ $0\}$. Denotamos por $\partial \mathbb{H}^{n}:=\left\{\left(x_{1}, x_{2}, \ldots, 0\right) \in \mathbb{R}^{n}\right\}$. Assim, $\mathbb{H}^{n} \backslash \partial \mathbb{H}^{n}=\left\{\left(x_{1}, x_{2}, \ldots, x_{n}\right) \in\right.$ $\left.\mathbb{R}^{n}: x_{n}>0\right\}$.

Definição 1.4 Considerando em $\mathbb{H}^{n}$ a topologia induzida do $\mathbb{R}^{n}$, diremos que $\mathcal{U} \subseteq \mathbb{H}^{n}$ é aberto em $\mathbb{H}^{n}$, se existir $V \subseteq \mathbb{R}^{n}$ aberto (no $\mathbb{R}^{n}$ ) tal que $\mathcal{U}=V \cap \mathbb{H}^{n}$.

Definição 1.5 Dado $\mathcal{U} \subseteq \mathbb{H}^{n}$ aberto de $\mathbb{H}^{n}$, diremos que $f: \mathcal{U} \rightarrow \mathbb{R}^{p}$ é diferenciável, se existir $V \subseteq \mathbb{R}^{n}$ aberto, com $\mathcal{U} \subset V$ e $\widetilde{f}: V \rightarrow \mathbb{R}^{p}$ diferenciável, tal que $\left.\widetilde{f}\right|_{\mathcal{U}} \equiv f$. Além disso, dado $x \in \mathcal{U}$, definimos a diferencial da aplicação $f$ em $x$ por $D f(x):=D \widetilde{f}(x)$.

Observação $1.13 D f(x)$ está bem definida e não depende da escolha de $\tilde{f}$.

Definição 1.6 Uma variedade $M$ de dimensão $m$ com bordo é um espaço topológico Hausdorff, com base enumerável (segundo enumerável) junto com uma coleção de aplicações (chamadas de cartas) $\phi_{\alpha}: V_{\alpha} \rightarrow \mathbb{H}^{m}$, com $\bigcup_{\alpha \in \Lambda} V_{\alpha}=M$ tais que:

(a) $\forall \alpha \in \Lambda, \phi_{\alpha}: V_{\alpha} \rightarrow \mathbb{R}^{m}$ é um homeomorfismo sobre o aberto (relativo) $\phi_{\alpha}\left(V_{\alpha}\right) \subset \mathbb{H}^{m}$;

(b) Se $V_{\alpha} \cap V_{\beta} \neq \emptyset$, então a aplicação (mudança de coordenadas) $\phi_{\beta} \circ \phi_{\alpha}^{-1}: \phi_{\alpha}\left(V_{\alpha} \cap V_{\beta}\right) \rightarrow$ $\phi_{\beta}\left(V_{\alpha} \cap V_{\beta}\right)$ é $C^{\infty}$-difeomorfismo, para todo $\alpha$ e $\beta$ em $\Lambda$. 
Definição 1.7 Dada uma variedade $M^{m}$ com bordo, um ponto $p \in M^{m}$ é chamado um ponto do bordo, se existir uma carta $(\mathcal{U}, \phi)$ de $p$ em $M$, tal que $\phi(p) \in \partial \mathbb{H}^{m}$. O conjunto de todos os pontos do bordo é chamado bordo de $\mathrm{M}$ e será denotado por $\partial M$. Além disso, denotaremos o conjunto dos pontos interiores de $M$ por $\stackrel{\leftrightarrow}{M}:=M \backslash \partial M$.

Proposição $1.14 O$ conjunto $\partial M$ está bem definido e é uma $(m-1)$-variedade suave (sem bordo). Além disso, a aplicação de inclusão $i_{\partial M}: \partial M \rightarrow M$ é suave.

Teorema 1.15 (Teorema de transversalidade para variedades com bordo) Sejam $M$ e $N$ variedades de dimensão $m$ e $n$, respectivamente, tais que $\partial M \neq \emptyset$ e $\partial N=\emptyset, Z \subset N$ é uma subvariedade sem bordo $r$-dimensional de $N$. Considere $f: M \rightarrow N \in C^{\infty} \operatorname{com} f \pitchfork Z$ e $\left.f\right|_{\partial M} \pitchfork Z$. Se $f^{-1}(Z) \neq \emptyset$, então $f^{-1}(Z)$ é uma subvariedade de $M$ de dimensão $[m-(n-r)]$, com bordo $\partial\left(f^{-1}(Z)\right)=\partial M \cap f^{-1}(Z)$.

Sendo $M^{m}$ uma variedade tal que $\partial M=\emptyset$ e $f: M \rightarrow \mathbb{R}$ uma função suave em $M$, diremos que um ponto $x_{0} \in M$ é um ponto crítico de $f$, se $D f\left(x_{0}\right) \equiv 0$. Um ponto crítico $x_{0}$ é chamado não-degenerado se, e somente se, a matriz $\left(\frac{\partial^{2} f}{\partial x_{i} \partial x_{j}}\left(x_{0}\right)\right)$ é não-singular, em que $\left(x_{1}, \cdots, x_{m}\right)$ é um sistema de coordenadas locais em uma vizinhança $\mathcal{U}$ de $x_{0}$. Podemos checar diretamente que tal condição não depende da escolha do sistema de coordenadas.

\subsection{Teoria de Morse para variedades com bordo}

A seguir apresentaremos alguns resultados sobre a Teoria de Morse para variedades com bordo conforme [ADD]. Em nossa exposição, objetivando uma apresentação mais clara dos principais resultados obtidos na Tese (os quais serão tratados nos capítulos 4 e 5), consideraremos $M$ uma variedade sem bordo, $g: M \rightarrow \mathbb{R}$ uma função suave e 0 um valor regular de $g$ tal que $M \cap\{g=0\} \neq \emptyset$. Assim segue que $M \cap\{g \leq 0\}$ (respectivamente, $M \cap\{g \geq 0\})$ é uma variedade com bordo $M \cap\{g=0\}$.

Definição 1.8 Seja $f: M \rightarrow \mathbb{R}$ uma função suave.

(a) Diremos que $x_{0}$ é um ponto crítico de $f_{\mid M \cap\{g \leq 0\}}$, se $x_{0}$ é um ponto crítico de $f_{\mid M \cap\{g<0\}}$ ou um ponto crítico de $f_{\mid M \cap\{g=0\}}$.

(b) Diremos que $x_{0} \in M \cap\{g=0\}$ é um ponto crítico correto de $f_{\mid M \cap\{g \leq 0\}}$, se $x_{0}$ é um ponto crítico de $f_{\mid M \cap\{g=0\}}$ e $x_{0}$ não é um ponto crítico de $f$.

(c) Diremos que $x_{0} \in M \cap\{g=0\}$ é um ponto crítico correto não-degenerado de $f_{\mid M \cap\{g \leq 0\}}$, se $x_{0}$ é um ponto crítico correto de $f_{\mid M \cap\{g \leq 0\}}$ e $x_{0}$ é um ponto crítico não-degenerado de $f_{\mid M \cap\{g=0\}}$.

Segue do item $(b)$ acima que, se $x_{0}$ é um ponto crítico correto de $f_{\mid M \cap\{g \leq 0\}}$, então $\nabla f\left(x_{0}\right) \neq 0$ e, além disso, existe $\tau\left(x_{0}\right) \neq 0$ tal que $\nabla f\left(x_{0}\right)=\tau\left(x_{0}\right) \nabla g\left(x_{0}\right)$. 
Para a definição abaixo identificaremos o vetor $\nabla f\left(x_{0}\right)$ com a sua projeção ortogonal no espaço $T_{x_{0}}(M \cap\{g \geq 0\})$ (resp. $\left.T_{x_{0}}(M \cap\{g \leq 0\})\right)$.

Definição 1.9 Seja $x_{0}$ um ponto crítico correto de $f_{\mid M \cap\{g \geq 0\}}$ (resp. $x_{0}$ um ponto crítico correto de $\left.f_{\mid M \cap\{g \leq 0\}}\right)$ :

(a) Diremos que $\nabla f\left(x_{0}\right)$ aponta para o interior de $M \cap\{g \geq 0\}$, se $\tau\left(x_{0}\right)>0$ (respectivamente, $M \cap\{g \leq 0\}$, se $\left.\tau\left(x_{0}\right)<0\right)$;

(b) Diremos que $\nabla f\left(x_{0}\right)$ aponta para o exterior de $M \cap\{g \geq 0\}$, se $\tau\left(x_{0}\right)<0$ (respectivamente, $M \cap\{g \leq 0\}$, se $\left.\tau\left(x_{0}\right)>0\right)$.

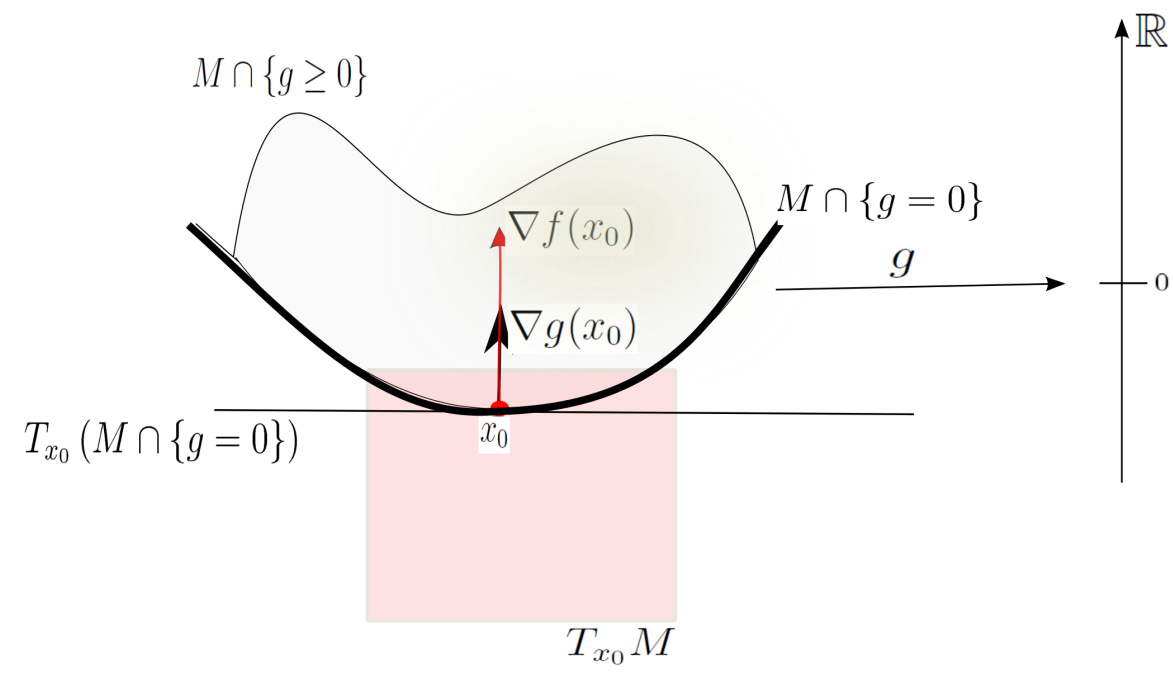

Definição 1.9 item (a): $\nabla f\left(x_{0}\right)$ aponta para o interior de $M \cap\{g \geq 0\}$

Definição 1.10 Seja $f: M \rightarrow \mathbb{R}$ uma função suave.

(a) Diremos que $f_{\mid M \cap\{g \leq 0\}}$ (respectivamente, $f_{\mid M \cap\{g \geq 0\}}$ ) é correta, se todos os pontos críticos de $f_{\mid M \cap g^{-1}(0)}$ são corretos.

(b) Diremos que $f_{\mid M \cap\{g \geq 0\}}$ (respectivamente, $f_{\mid M \cap\{g \leq 0\}}$ ) é uma função Morse correta, se $f_{\mid M \cap\{g>0\}}$ (respectivamente, $f_{\mid M \cap\{g<0\}}$ ) admite somente pontos críticos não-degenerados e se $f_{\mid M \cap\{g=0\}}$ admite somente pontos críticos corretos não-degenerados.

Proposição 1.16 Sejam $M$ uma variedade e $g: M \rightarrow \mathbb{R}$ uma função suave tal que 0 é valor regular de $g$. O conjunto de funções $f: M \rightarrow \mathbb{R}$ suaves tais que $f_{\mid M \cap\{g \leq 0\}}$ e $f_{\mid M \cap\{g \geq 0\}}$ é uma função Morse correta é denso em $C^{\infty}(M, \mathbb{R})$.

Ainda considerando $f: M \rightarrow \mathbb{R}$ uma função suave em $M$. Denotaremos por

$$
M^{c}:=f^{-1}(-\infty, c]=\{x \in M: f(x) \leq c\} .
$$


Exemplo 1.17 A seguir $M$ representa um disco fechado no plano $x O y$ com dois discos abertos removidos do seu interior, $h(x, y):=y$ denota a "função altura no plano".

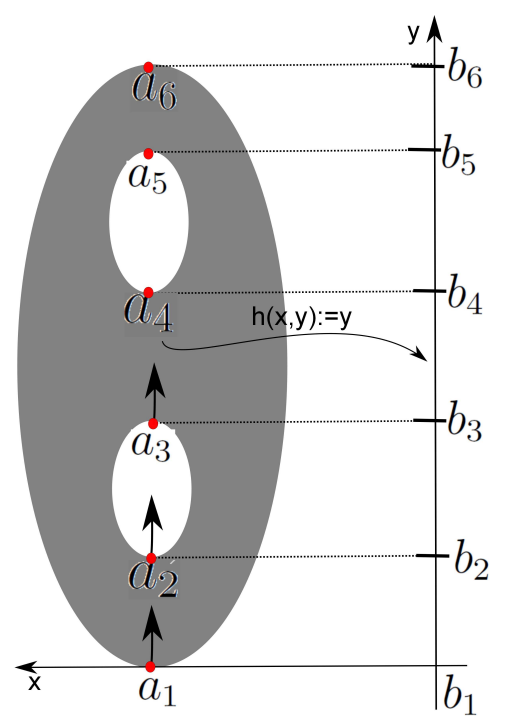

Então,

(a) $M^{c}$ é vazio, se c $<0$;

(b) $M^{c}$ é homeomorfo a um disco, se $b_{1}<c<b_{2}$;

(c) $M^{c}$ é homeomorfo a um anel, se $b_{3}<c<b_{4}$;

Os resultados a seguir são análogos aos apresentados em [Mil2] para o caso de variedades sem bordo. No que segue consideraremos $M$ uma variedade com bordo e $f: M \rightarrow \mathbb{R}$ uma função suave.

Lema 1.18 [HL, Lema 3.1.4, pg. 333] Seja $a<b$ e suponha que o conjunto $f^{-1}([a, b])$, consistindo de todos os pontos $x \in M$ com $a \leq f(x) \leq b$ seja compacto, e não contenha pontos críticos de $f$. Então $M^{a}$ é homeomorfa a $M^{b}$. Além disso, $M^{a}$ é um retrato por deformação de $M^{b}$. Assim, temos que a aplicação inclusão $M^{a} \subset M^{b}$ é uma equivalência de homotopia.

Lema 1.19 [HL, Lema 3.1.5, pg. 333] Seja $a<b$ e suponha que $f^{-1}([a, b])$ seja compacto e que $f^{-1}([a, b[)$ não contenha qualquer ponto crítico de $f$. Então, $\{x \in M: f(x)<a\}$ é um retrato por deformação de $\{x \in M: f(x)<b\}$, e os dois espaços são difeomorfos.

Lema 1.20 [HL, Lema 3.1.6, pg. 333] Suponha que $x_{0}$ seja um ponto crítico não-degenerado de $f_{\mid M \backslash \partial M}$ com indice $\lambda$. Considere $f\left(x_{0}\right)=c$ e suponha que para certo $\epsilon_{0}>0$ o conjunto $f^{-1}\left(\left[c-\epsilon_{0}, c+\epsilon_{0}\right]\right)$ seja compacto, e não contenha pontos críticos de $f$ distintos de $x_{0}$. Então, para todo $\epsilon$ suficientemente pequeno, temos que o conjunto $M^{c+\epsilon}$ tem o mesmo tipo de homotopia de $M^{c-\epsilon}$ com uma $\lambda$-célula atachada. 
A seguir, apresentaremos um importante resultado sobre pontos críticos de funções em variedades com bordo. Este resultado foi provado em [AD].

Lema 1.21 [AD, Lema 6.1, pg. 9] Seja $M$ uma variedade compacta e com bordo e $f: M \rightarrow$ $\mathbb{R}$ uma função suave. Vamos supor que $x_{0} \in \partial M$ é um ponto crítico correto de $f$. Considere $f\left(x_{0}\right)=c$ e suponha que exista $\epsilon>0$ tal que o conjunto $f^{-1}([c-\epsilon, c+\epsilon])$ não contenha outros pontos críticos de $f$ distintos de $x_{0}$. Se $x_{0}$ é um ponto crítico cujo gradiente de $f$ em $x_{0}$ aponta para o exterior, então $\left.\left.f^{-1}(]-\infty, c+\epsilon\right]\right)$ é homeomorfo a $\left.\left.f^{-1}(]-\infty, c-\epsilon\right]\right)$.

Com o objetivo de ilustrar os Lemas 1.20 e 1.21 consideraremos o exemplo 1.17 - página 13 , em que $a_{i}, i=1, \cdots, 6$ são os pontos críticos de $h(x, y):=y$.

Se $b_{3}<c<b_{4}$, temos que $M^{b_{3}} \simeq M^{c}$, mas $M^{b_{3}} \nsim M^{c}$ (observe que, neste caso, $\nabla h\left(a_{3}\right)$ aponta para o interior de $M$ ).
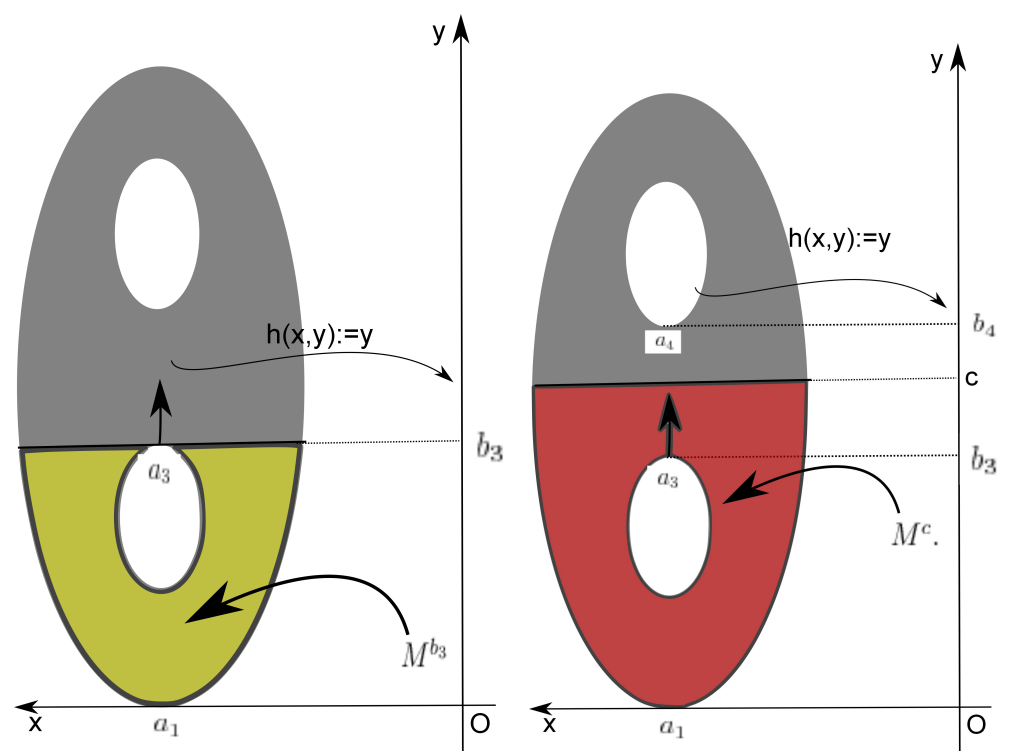

Por outro lado, se $b_{2}<c<b_{3}$, então, temos que $M^{b_{2}} \sim M^{c}$ (observe que, neste caso, $\nabla h\left(a_{2}\right)$ aponta para o exterior de $\left.M\right)$. 

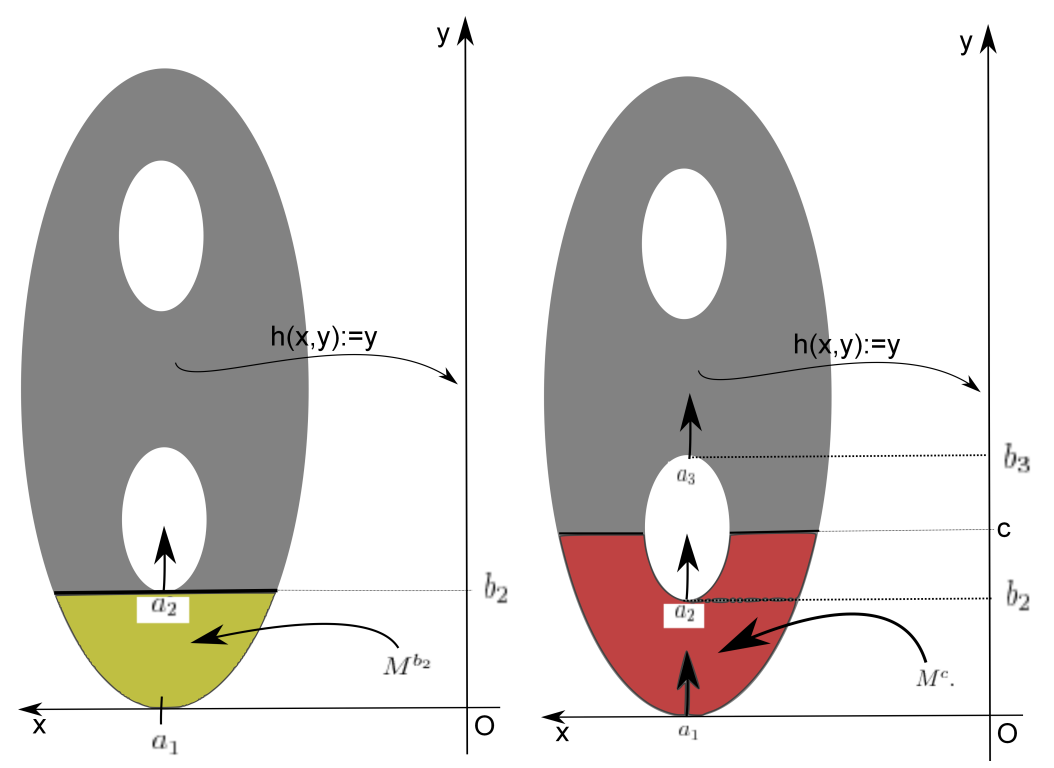

\subsubsection{Variedades com corners}

A seguir apresentaremos uma outra classe de variedades denominada de "variedades com corners". Tais variedades aparecerão naturalmente nos estudos desenvolvidos no Capítulo 5.

Definição 1.11 [HL, definição 3.2.1, pg. 334] Sejam $E^{p}=\left\{\left(x_{1}, \cdots, x_{p-1}, x_{p}\right) \in \mathbb{R}^{p}\right.$ : $\left.x_{p-1} \geq 0, x_{p} \geq 0\right\}, M$ um espaço topológico Hausdorff com base enumerável e $\left(\mathcal{U}_{\alpha}, \varphi_{\alpha}\right)_{\alpha \in A}$ uma família de pares de abertos $\mathcal{U}_{\alpha}$ de $M$ e de homeomorfismos $\varphi_{\alpha}: \mathcal{U}_{\alpha} \rightarrow V_{\alpha}$, $V_{\alpha}$ é um aberto de $E^{p}$, tais que:

(a) $\bigcup_{\alpha \in A} \mathcal{U}_{\alpha}=M$

(b) para todos $(\alpha, \beta) \in A^{2}$ tal que $\mathcal{U}_{\alpha} \cap \mathcal{U}_{\beta} \neq \emptyset$ a aplicação $\varphi_{\alpha, \beta}: \varphi_{\alpha}\left(\mathcal{U}_{\alpha} \cap \mathcal{U}_{\beta}\right) \rightarrow \varphi_{\beta}\left(\mathcal{U}_{\alpha} \cap\right.$ $\left.\mathcal{U}_{\beta}\right)$, em que $z \in \varphi_{\alpha}\left(\mathcal{U}_{\alpha} \cap \mathcal{U}_{\beta}\right)$ faz corresponder $\varphi_{\beta}\left(\varphi_{\alpha}^{-1}(z)\right)$, é diferenciável, isto é, existe uma aplicação de uma vizinhança aberta de $\varphi_{\alpha}\left(\mathcal{U}_{\alpha} \cap \mathcal{U}_{\beta}\right)$ de $\mathbb{R}^{p}$ numa vizinhança aberta de $\varphi_{\beta}\left(\mathcal{U}_{\alpha} \cap \mathcal{U}_{\beta}\right)$ de $\mathbb{R}^{p}$ que induz $\varphi_{\alpha, \beta}$ que é diferenciável;

(c) para todo aberto $\mathcal{U}$ de $M$ e todo homeomorfismo $\varphi: \mathcal{U} \rightarrow E$, onde $E$ é um subconjunto de $\mathbb{R}^{p}$, temos que, para todo par $\left(\mathcal{U}_{\alpha}, \varphi_{\alpha}\right)$ tal que $\mathcal{U} \cap \mathcal{U}_{\alpha} \neq \emptyset$ a aplicação

$$
\varphi \circ \varphi_{\alpha}^{-1}: \varphi_{\alpha}\left(\mathcal{U} \cap \mathcal{U}_{\alpha, \beta}\right) \rightarrow \varphi\left(\mathcal{U} \cap \mathcal{U}_{\alpha, \beta}\right)
$$

é um difeomorfismo.

Uma tal família $\left(\mathcal{U}_{\alpha}, \varphi_{\alpha}\right)$ é chamada um atlas com corner de dimensão $p$ de $M$ e, neste caso, diremos que $M$ é uma variedade com corner de dimensão $p$. Além do mais, diremos que $\varphi_{\alpha}$ é uma carta da variedade com corner $e$ que $\varphi_{\alpha, \beta}$ é a aplicação de transição da carta $\varphi_{\alpha}$ para a carta $\varphi_{\beta}$. 
Análogo ao caso de variedades com bordo, podemos definir na classe de variedade com corners a noção de subvariedade. Nesta classe um isomorfismo é chamado um difeomorfismo.

Os pontos $x \in M$ em que existe uma vizinhança aberta $\mathcal{U}$ de $M$ e um difeomorfismo $\varphi$ de $\mathcal{U}$ em $B^{+}=\left\{x \in \mathbb{R}^{p}:\|x\|<\epsilon\right.$ e $\left.x_{p} \geq 0\right\}, \varphi(x)=0$, são os pontos do bordo de $M$, que também denotaremos por $\partial M$.

Os pontos $x \in M$ em que existe uma vizinhança aberta $\mathcal{U}$ de $M$ e um difeomorfismo $\varphi$ de $\mathcal{U}$ em $D^{+}=\left\{x \in \mathbb{R}^{p}:\|x\|<\epsilon, x_{p} \geq 0\right.$ e $\left.x_{p-1} \geq 0\right\}, \varphi(x)=0$, são os pontos de corner de $M$. Denotaremos tal conjunto por ${ }_{\partial} M$.

Os pontos de $\stackrel{\circ}{M}=M \backslash\left(\partial M \cup{ }_{\partial} M\right)$ são chamados pontos interiores de $M$, ou seja, os pontos $x \in M$ tais que existe uma vizinhança aberta $\mathcal{U}$ de $M$ e um difeomorfismo $\varphi$ de $\mathcal{U}$ em $B=\left\{x \in \mathbb{R}^{p}:\|x\|<\epsilon\right\}, \varphi(x)=0$.

Exemplo 1.22 Qualquer cubo fechado em $\mathbb{R}^{n}$ é uma n-variedade com corner.

Veremos a seguir um exemplo interessante de variedade com corner como uma aplicação do Lema de seleção da curva local.

Aplicação 1.23 Seja $f: \mathcal{U} \rightarrow \mathbb{C}$ uma função polinomial holomorfa, $\mathcal{U}$ uma vizinhança aberta da origem $0 \in \mathbb{C}^{n}$, com $f(0)=0$. Seja $B_{\epsilon} \subset \mathcal{U}$ uma bola fechada centrada na origem de raio $\epsilon>0$ e denote por $S_{\epsilon}=\partial B_{\epsilon}$. Segue da desigualdade de Eojasiewicz para funções analiticas reais que podemos escolher $\epsilon$ suficientemente pequeno tal que $\Sigma_{|f|^{2}} \subset V$, em que $V=f^{-1}(0) \cap \mathcal{U}$. Sendo $|f|^{2}$ uma função polinomial e $S_{\epsilon}$ uma variedade semi-algébrica, segue que a restrição de $|f|^{2}$ a $S_{\epsilon}$ tem eventualmente um valor crítico em 0 . Neste caso, podemos provar que $0 \in \mathbb{R}$ é um valor crítico isolado de $|f|^{2}: S_{\epsilon} \rightarrow \mathbb{R}$.

DemonstraÇão: De fato, seja $\left\{y_{k}\right\} \subset \mathbb{R}$ uma sequência de valores críticos $y_{k} \neq 0$ tal que $y_{k} \rightarrow 0$. Logo, existe uma sequência de pontos críticos $\left\{x_{k}\right\} \subset S_{\epsilon} \backslash K_{\epsilon}$ tal que $|f|^{2}\left(x_{k}\right)=y_{k}$. A menos de subsequência podemos assumir que $x_{k} \rightarrow x \in K_{\epsilon}$. Pelo Lema de seleção da curva local existe $\delta>0$ e uma curva analítica $\varphi:[0, \delta) \rightarrow S_{\epsilon} \backslash K_{\epsilon}$ tal que $\varphi(0)=x$ e, $\forall t \in(0, \delta)$ temos que $\varphi(t) \subset S_{\epsilon} \backslash K_{\epsilon}$ é ponto crítico de $|f|^{2}$. Logo, existe um $\lambda(t) \neq 0$ tal que $\nabla|f|^{2}(t)=\lambda(t) \varphi(t), t \in(0, \delta)$. Assim,

$$
\begin{aligned}
\frac{d}{d t}\left(|f|^{2} \varphi(t)\right) & =\left\langle\nabla|f|^{2}(t), \varphi^{\prime}(t)\right\rangle \\
& =\lambda(t)\left\langle\varphi(t), \varphi^{\prime}(t)\right\rangle \\
& =\frac{\lambda(t)}{2} \frac{d}{d t}\left(\|\varphi(t)\|^{2}\right) \\
& =\frac{\lambda(t)}{2} \frac{d}{d t}\left(\epsilon^{2}\right)=0
\end{aligned}
$$

Portanto, $|f|^{2}(\varphi(t))=|f|^{2}(x)=0$ o que é um absurdo pois $\varphi(t) \subset S_{\epsilon} \backslash K_{\epsilon}$ para $t \neq 0$.

Consequentemente, existe um $0<\delta_{1} \ll \epsilon$ (muito pequeno comparado com $\epsilon$ ) tal que $|f|^{2}$ não possui valores críticos em $\left(0, \delta_{1}\right]$. Logo, para todo $\delta_{2} \in\left(0, \delta_{1}\right]$ o conjunto $T=\{x \in$ 
$\left.B_{\epsilon}:|f|^{2}=\delta_{2}\right\}$ é uma variedade com bordo e o conjunto $C=\left\{x \in B_{\epsilon}:|f|^{2} \geq \delta_{2}\right\}$ é uma variedade com corner. Seu bordo é constituído pelos pontos $\partial C=\left\{x \in S_{\epsilon}:|f|^{2}>\delta_{2}\right\} \cup\{x \in$ $\left.\stackrel{\circ}{B}_{\epsilon}:|f|^{2}=\delta_{2}\right\}$. Seus pontos de corner são os pontos ${ }_{\partial}(C)=T \cap S_{\epsilon}$.

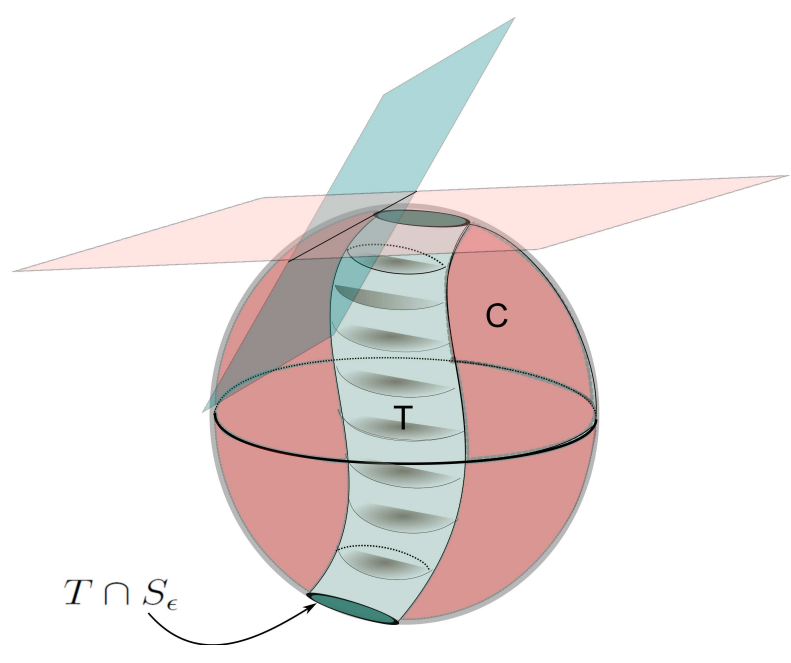

\subsubsection{Teorema de fibração para variedades com bordo}

Abaixo apresentaremos a definição de fibração suave localmente trivial, em seguida, um teorema que utilizaremos com bastante frequência ao longo do texto e que fornece condições suficientes para que se tenha uma fibração suave localmente trivial.

Definição 1.12 Uma $C^{\infty}$-fibração localmente trivial (ou fibração suave) é uma quadra $(M, N, F, \phi)$, em que $M, N, F$ são variedades, $N$ conexa, tal que:

(a) $\phi: M \rightarrow N$ é uma submersão, sobrejetora, suave $e$

(b) $\forall b \in N$, existe uma vizinhança $\mathcal{U}_{b} \subseteq N$ e um difeomorfismo $h_{b}: \mathcal{U}_{b} \times F \rightarrow \phi^{-1}\left(\mathcal{U}_{b}\right)$ (esse difeomorfismo é chamado de trivialização local); tal que $\phi \circ h_{b}=\pi_{1}$, em que $\pi_{1}$ é a projeção na primeira coordenada. Ou seja, tal que o seguinte diagrama seja comutativo

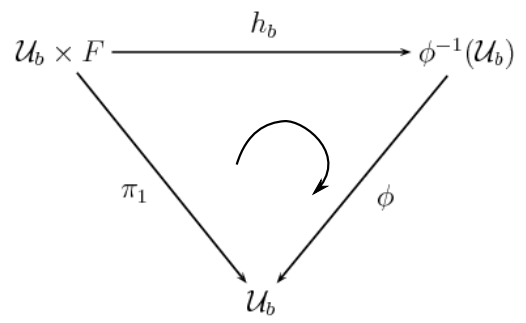

\section{Observação 1.24}


(a) Como $N$ é conexa, temos que $\pi_{1}^{-1}(y)$ é uma cópia difeomorfa de $F$, para todo $y \in N$.

(b) $S e \mathcal{U}_{b}=N$, diremos que o fibrado é trivial.

(c) A definição acima pode ser adaptada para o caso de variedades com bordo. Por exemplo, se $\partial M \neq \emptyset$, então trocamos o item (a) pela seguinte condição: $\phi_{\mid M}$ e $\phi_{\mid \partial M}$ são submersões, sobrejetivas e suaves.

Definição 1.13 Sejam $M, N, B$ variedades. Dizemos que duas fibrações suaves localmente triviais $F: M \rightarrow B$ e $G: N \rightarrow B$ são $C^{k}$-equivalentes (ou $C^{k}$-isomorfas), $k=0,1 \cdots, \infty$, se existe um $C^{k}$-difeomorfismo $h: M \rightarrow N$ tal que o seguinte diagrama é comutativo:

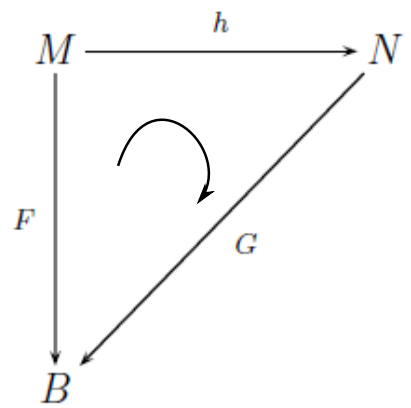

Exemplo 1.25 A aplicação $\pi: S^{2 n+1} \rightarrow \mathbb{C P}^{n}$ dada por $\pi(x):=[x]$ é fibração suave localmente trivial com fibra $S^{1}$. No caso $n=1$, sabendo que $\mathbb{C P}^{1}$ é difeomorfo à esfera $S^{2}$, temos a bem conhecida fibração de Hopf.

Teorema 1.26 (Teorema de fibração de Ehresmann para variedade com bordo) Sejam M uma variedade com bordo, $N$ uma variedade conexa fechada e $F: M \rightarrow N$ sobrejetiva suave e própria, tal que $F_{\mid M}$ e $F_{\mid \partial M}$ são submersões sobrejetivas. Então, $F$ é uma fibração suave localmente trivial. 


\section{Capítulo 2}

\section{Classes Especiais de Polinômios}

\section{Sumário}

2.1 Polinômios Newton não-degenerados no infinito . . . . . . . 19

2.2 Polinômios Tame . . . . . . . . . . . . . . 22

2.3 Polinômios Quasitame e $M$-Tame . . . . . . . . . . . 24

2.4 Polinômios Semitame .................. 28

Neste capítulo apresentaremos algumas classes "especiais" de polinômios, que admitem uma estrutura de fibração em esferas de raios suficientemente grandes (Teorema 3.1 - página 32) e que abriram caminho para um estudo do comportamento assintótico das fibras no infinito, a partir de ferramentas e técnicas de diversas áreas da Matemática. Podemos citar, por exemplo, a classe dos polinômios semitame (definição 2.10 - página 28) introduzidos por András Némethi e Alexandru Zaharia [NZ1], a qual contém os polinômios tame (definição 2.3 - página 22), introduzidos por S. Broughton [Br2] e os polinômios quasitame (definição 2.5 - página 24) introduzidos por András Némethi [NZ1], dentre outras classes de polinômios que serão apresentadas ao longo do texto.

Nossas principais referências são os artigos [Br1, Br2, NZ1, Pa].

\subsection{Polinômios Newton não-degenerados no infinito}

Nesta seção, definiremos os polinômios Newton não-degenerados no infinito com base no artigo [NZ1].

Seja $f=\sum_{\nu \in \mathbb{N}^{*}} a_{\nu} z^{\nu} \in \mathbb{C}\left[z_{1}, \cdots, z_{n}\right]$ polinômio, em que $\nu:=\left(\nu_{1}, \cdots, \nu_{n}\right)$ e $z^{\nu}:=z_{1}^{\nu_{1}} \cdots \cdots z_{n}^{\nu_{n}}$, como usualmente.

Definição 2.1 Assim definimos e denotamos por:

(a) $\operatorname{supp}(f):=\left\{\nu \in \mathbb{N}^{*}: a_{\nu} \neq 0\right\} \subseteq \mathbb{R}^{n}$, chamado de suporte de $f$; 
(b) $\overline{\operatorname{supp}}(f):=$ fecho convexo de $\operatorname{supp}(f)$;

(c) $\widetilde{\Gamma}_{-}(f):=$ fecho convexo de $\{0\} \cup \operatorname{supp}(f)$;

(d) $\widetilde{\Gamma}(f):=$ a união das faces fechadas do poliedro $\widetilde{\Gamma}_{-}(f)$ que não contém a origem é chamado bordo de Newton no infinito do polinômio $f$.

Seja $\Delta$ face fechada do poliedro $\widetilde{\Gamma}(f)$, denotamos por $f_{\Delta}$ o polinômio $\sum_{m \in \Delta} a_{m} z^{m}$.

(e) Diremos que $f$ é não degenerado em $\Delta$, se o sistema de equações

$$
\frac{\partial f_{\Delta}}{\partial z_{1}}=\cdots=\frac{\partial f_{\Delta}}{\partial z_{n}}=0
$$

não tem solução em $(\mathbb{C} \backslash\{0\})^{n}$. Diremos que $f$ é Newton não degenerado no infinito, se $f$ é não degenerado em todas as faces fechadas $\Delta$ de $\widetilde{\Gamma}(f)$.

(f) Diremos que $f$ é conveniente, se a interseção do supp $(f)$ com cada eixo coordenado é não vazia.

Definição 2.2 Dizemos que uma face fechada $\Delta$ do $\overline{\operatorname{supp}}(f)$ é ruim, se:

(a) a "menor" subvariedade afim que contém $\Delta$ (menor no sentido da dimensão), passa pela origem;

(b) existe um hiperplano $H \subseteq \mathbb{R}^{n}$ com equação $a_{1} x_{1}+\cdots+a_{n} x_{n}=0$, (em que $x_{1}, \cdots, x_{n}$ são coordenadas em $\mathbb{R}^{n}$ ) tal que

(i) existem $i$ e $j$ com $a_{i}<0$ e $a_{j}>0$;

(ii) $H \cap \overline{\operatorname{supp}}(f)=\Delta$.

Com essas notações e definições podemos introduzir as seguintes classes de polinômios.

(a) $\mathcal{N} \mathcal{N}:=\{f$ é Newton não degenerado no infinito com $f(0)=0\}$;

(b) $\mathcal{N C}:=\{f \in \mathcal{N} \mathcal{N}: f$ é conveniente $\}$;

(c)

$$
\mathcal{N N}_{0}:=\left\{\begin{array}{c}
f \in \mathcal{N N}: f \text { tem somente singularidades isoladas em } \mathbb{C}^{n} \backslash f^{-1}(0) \\
\text { e } \overline{\operatorname{supp}}(f) \text { não possui faces ruins }
\end{array}\right\} .
$$

Exemplo 2.1 (Exemplo do Broughton) Seja $f: \mathbb{C}^{2} \rightarrow \mathbb{C}$, definido por $f=x+y x^{2}$.
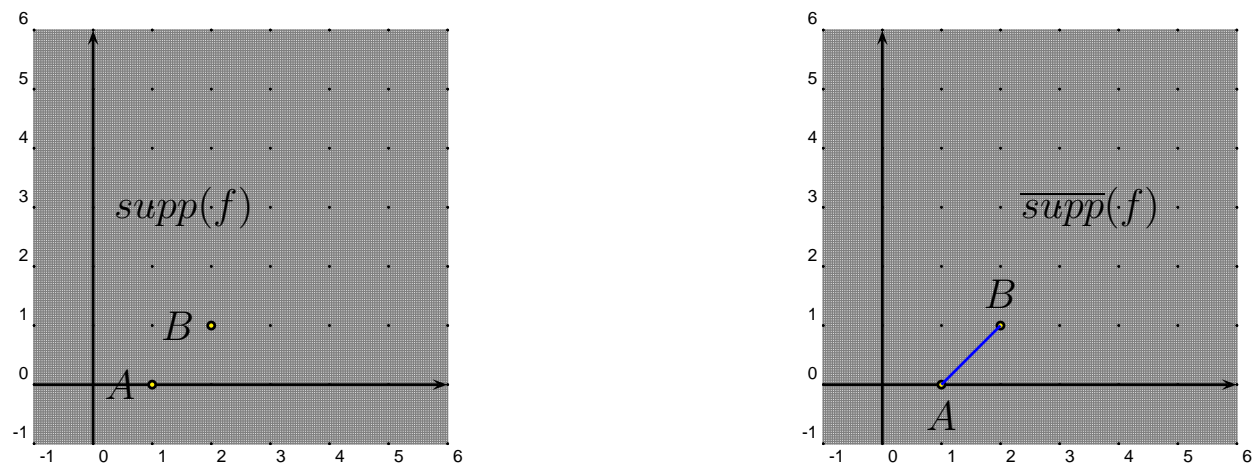

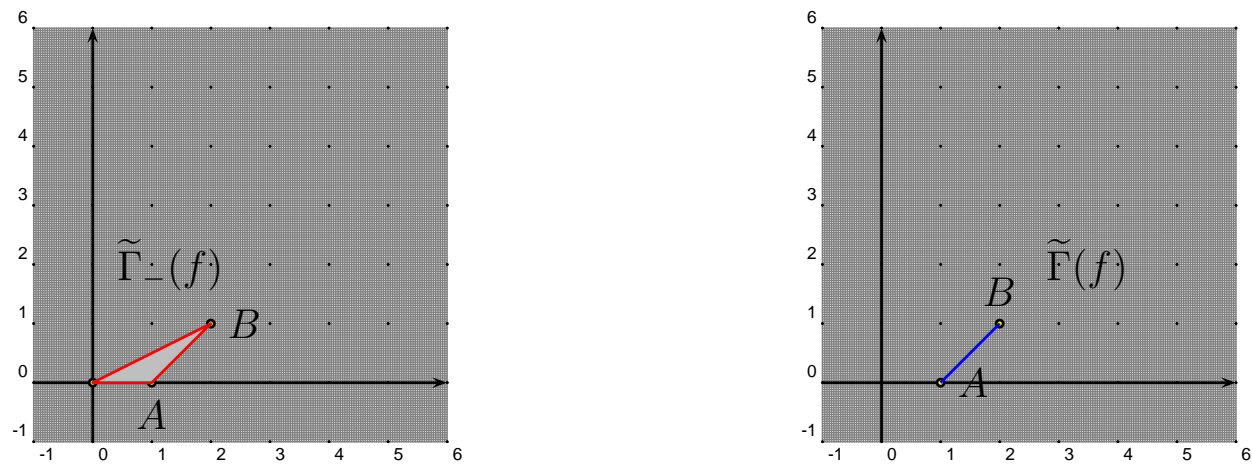

Vamos verificar a seguir algumas propriedades de $f$ relativas ao poliedro de Newton no infinito. Pelo diagrama acima, é suficiente considerar as seguintes faces $\Delta_{1}=(2,1), \Delta_{2}=$ $(1,0)$ e $\Delta_{3}=$ segmento de reta ligando os pontos $(1,0)$ e $(2,1)$.

Considerando $f_{\Delta_{1}}=y x^{2}$, temos que o sistema

$$
\begin{aligned}
& \frac{\partial f_{\Delta_{1}}}{\partial x}=0 \\
& \frac{\partial f_{\Delta_{1}}}{\partial y}=0
\end{aligned}
$$

admite a solução $(0, y) \not \subset(\mathbb{C} \backslash\{0\})^{2}$, assim a face $\Delta_{1}$ é não degenerada.

Considerando $f_{\Delta_{2}}=x$, claramente o sistema de equações

$$
\begin{aligned}
& \frac{\partial f_{\Delta_{2}}}{\partial x}=0 \\
& \frac{\partial f_{\Delta_{2}}}{\partial y}=0
\end{aligned}
$$

não possui solução.

Por fim, sendo $f_{\Delta_{3}}=x+y x^{2}$, temos que o sistema

$$
\begin{aligned}
& \frac{\partial f_{\Delta_{3}}}{\partial x}=0 \\
& \frac{\partial f_{\Delta_{3}}}{\partial y}=0
\end{aligned}
$$

também não possui solução.

Portanto, $f$ é Newton não degenerado no infinito e como $f(0)=0$ temos que $f \in \mathcal{N} \mathcal{N}$. Além disso, $f \in \mathcal{N N}_{0}$, pois $f$ não possui face ruim, e $f \notin \mathcal{N C}$ pois supp $(f) \cap O y=\emptyset$.

Exemplo 2.2 Seja $f: \mathbb{C}^{2} \rightarrow \mathbb{C}, f=x y^{2}+x y^{4}$. É fácil verificar que $\Sigma_{f}=\{(0, i),(0,-i)\} \cup$ $\{(x, 0): x \in \mathbb{C}\}$, logo $f$ tem somente singularidades isoladas em $\mathbb{C}^{2} \backslash f^{-1}(0)$. O bordo de Newton no infinito de $f$ é dado por 


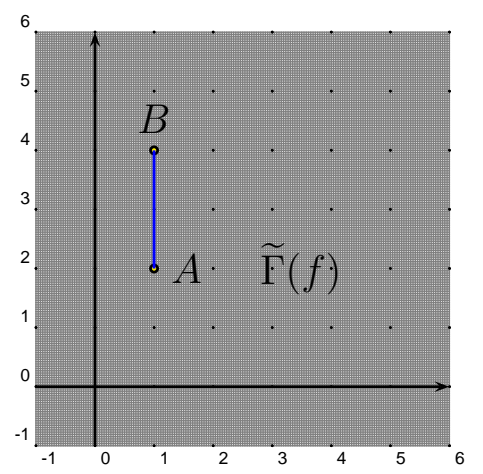

Considere as faces $\Delta_{1}=(1,2), \Delta_{2}=(1,4)$ e $\Delta_{3}=$ segmento de reta ligando os pontos $(1,2)$ e $(1,4)$. Logo, $f_{\Delta_{1}}=x y^{2}, f_{\Delta_{2}}=x y^{4}$ e $f_{\Delta_{3}}=f$ e como $\overline{\operatorname{supp}}(f)=\Delta_{3}$ não está contido em qualquer reta que passa pela origem, segue que $f \in \mathcal{N} \mathcal{N}$ e $f \in \mathcal{N N}_{0}$.

\subsection{Polinômios Tame}

A seguir relembraremos a definição e alguns resultados básicos obtidos por Broughton em $[\mathrm{Br} 1]$.

Sejam $f: \mathbb{C}^{n} \rightarrow \mathbb{C}$ uma função polinomial e $\left\{z_{k}\right\} \subseteq \mathbb{C}^{n}$ uma sequência tal que:

$$
\lim _{k \rightarrow \infty}\left\|z_{k}\right\|=+\infty \text { e } \lim _{k \rightarrow \infty} \bar{\nabla} f\left(z_{k}\right)=0 .
$$

Definição 2.3 Dada $f$ como acima, diremos que $f$ é tame, se não existe uma sequência $\left\{z_{k}\right\} \subseteq \mathbb{C}^{n}$ tal que a Condição 2.1 é satisfeita.

Denotaremos por $\mathcal{T}$ a classe de polinômios tame.

Observação 2.3 (a) Os polinômios tame foram introduzidos por Broughton [Br2] e o termo tame é usado porque a curva integral do campo de vetores $\xi(z)=\frac{\frac{(B f(z)}{\nabla \nabla f(z) \|^{2}}}{\| e m}$ comportamento moderado.

(b) Decorre da definição que um polinômio tame tem um número finito de pontos críticos.

Definição 2.4 Seja $f: \mathbb{C}^{n} \rightarrow \mathbb{C}$ uma função polinomial holomorfa tendo somente pontos críticos isolados e $c \in \mathbb{C}$. Sejam $P_{1}, \cdots, P_{r}$ os pontos críticos de $f$ em $f^{-1}(c)$ e $\mu^{c}(f):=$ $\sum_{i=1}^{r} \mu_{P_{i}}(f)$, em que $\mu_{P_{i}}(f)$ é o o número de Milnor local de $f$. Definimos o o número total de Milnor de $f$ como sendo $\mu(f):=\sum_{c \in \mathbb{C}} \mu^{c}(f)$.

Observação 2.4 É possível mostrar que, se $f: \mathbb{C}^{n} \rightarrow \mathbb{C}$ é uma função polinomial holomorfa com somente singularidades isoladas, então

$$
\mu(f)=\operatorname{dim}_{\mathbb{C}} \frac{\mathbb{C}\left[z_{1}, \cdots, z_{n}\right]}{\left(\frac{\partial f}{\partial z_{1}}, \cdots, \frac{\partial f}{\partial z_{n}}\right)},
$$


em que $\left(\frac{\partial f}{\partial z_{1}}, \cdots, \frac{\partial f}{\partial z_{n}}\right)$ denota o ideal gerado pelas derivadas parciais no anel de polinômios $\mathbb{C}\left[z_{1}, \cdots, z_{n}\right]$.

Sejam $f: \mathbb{C}^{n} \rightarrow \mathbb{C}$ uma função polinomial e $\Sigma_{f}$ o conjunto de pontos críticos de $f$. Sendo $f \in \mathcal{T}$, temos que $\Sigma_{f}$ é finito. Com isto, existe $R_{0} \gg 1$, tal que $\forall R \geq R_{0}, \Sigma_{f} \subset \stackrel{\circ}{B}_{R}$, em que $\stackrel{\circ}{B}_{R}$ denota a bola aberta de centro na origem e raio $R$. Portanto, a aplicação

$$
R \frac{\nabla f}{\|\nabla f\|}: S_{R}^{2 n-1} \rightarrow S_{R}^{2 n-1}
$$

está bem definida e tem um grau topológico que denotaremos por $\operatorname{deg}_{\infty}(\nabla f)$.

Considerando $f\left(\Sigma_{f}\right)=\left\{c_{1}, \cdots, c_{k}\right\}$, em [Br1, Proposição 2.1, pg. 220] foi provado $\mu(f):=\sum_{i=1}^{k} \mu^{c_{i}}(f)=\operatorname{deg}_{\infty}(\nabla f)$.

De forma geral, dados dois espaços topológicos $X$ e $Y$, diremos que uma aplicação $p$ : $X \rightarrow Y$ é própria em $y \in Y$, se existir uma vizinhança $\mathcal{U}$ de $y$ em $Y$, tal que $p: p^{-1}(\mathcal{U}) \rightarrow \mathcal{U}$ é própria. Logo $f: \mathbb{C}^{n} \rightarrow \mathbb{C}$ é tame se, e somente se, $\nabla f: \mathbb{C}^{n} \rightarrow \mathbb{C}^{n}$ é própria na origem.

Com as definições e notações acima, foi provada uma caracterização dos polinômios tame em termos do número de Milnor, como a seguir.

Proposição 2.5 [Br1, Proposição 3.1, pg. 225] Sejam $f: \mathbb{C}^{n} \rightarrow \mathbb{C}$ uma função polinomial e $f^{w}(z)=f\left(z_{1}, \cdots, z_{n}\right)-\left(w_{1} z_{1}+\cdots+w_{n} z_{n}\right)$, em que $z=\left(z_{1}, \cdots, z_{n}\right), w=\left(w_{1}, \cdots, w_{n}\right) \in \mathbb{C}^{n}$. Então, $f \mathcal{T}$ se, e somente se, $\mu(f)<\infty$ e $\mu\left(f^{w}\right)=\mu(f)$ para todo $w \in \mathbb{C}^{n}$ suficientemente pequeno.

Demonstração: Inicialmente vamos fazer a prova da volta.

$((\Leftarrow))$ Suponha que $\mu\left(f^{w}\right)=\mu(f)<\infty$ para todo $w$ suficientemente pequeno. Considere os pontos de $(\nabla f)^{-1}(0)$ contidos em bolas fechadas disjuntas, e denote por $B$ a união destas bolas. Para $w$ suficientemente pequeno, temos que $\nabla f^{w}=\nabla f-w$, assim $\left\|\nabla f^{w}(z)-\nabla f(z)\right\|=\|w\|<\|\nabla f(z)\|, z \in \partial B$. Portanto, os campos $\nabla f^{w}$ e $\nabla f$ são homotópicos e, por hipótese, $\operatorname{deg}_{\infty}\left(\nabla f^{w}\right)=\mu\left(f^{w}\right)=\mu(f)<\infty$ para todo $w$ suficientemente pequeno. Logo, $(\nabla f)^{-1}$ é própria na origem. Assim, temos que $f$ é tame.

$((\Rightarrow))$ Sendo $f$ tame, segue que $\nabla f$ é própria na origem e temos que $(\nabla f)^{-1}(w) \subseteq \stackrel{\circ}{B}$ para todo $w$ suficientemente pequeno, em que $\stackrel{\circ}{B}$ denota a bola aberta. Portanto, $\mu\left(f^{w}\right)=$ $\mu(f)<\infty$ pela [Br1, Proposição 2.1, item i, pg. 220].

Exemplo 2.6 Sendo $f(x, y)=x^{2} y+x$ e considerando $w=(a, b)$, temos que $\mu\left(f^{w}\right)=0$, $a \neq-1, b=0$ e $\mu\left(f^{w}\right)=2, b \neq 0$ Assim, segue da Proposição anterior que $f$ não é tame. 


\subsection{Polinômios Quasitame e $M$-Tame}

A seguir apresentaremos a classe de polinômios quasitame introduzida por András Némethi em [NZ1].

Definição 2.5 Seja $f: \mathbb{C}^{n} \rightarrow \mathbb{C}$ uma função polinomial. Definimos os polinômios quasitame como sendo:

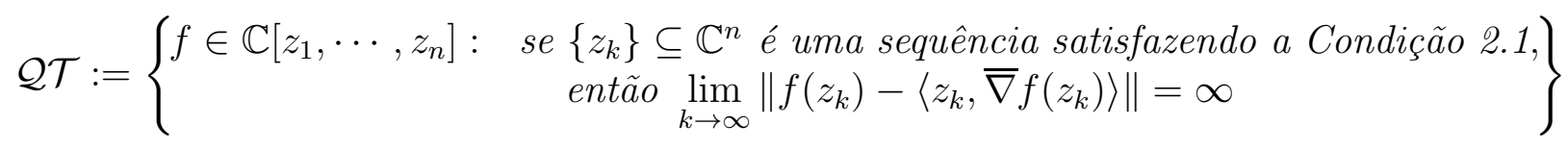

Observação 2.7 Se $f \in \mathcal{T}$, então $f \in \mathcal{Q T}$. De fato, observe que se $f \in \mathbb{C}\left[z_{1}, \cdots, z_{n}\right]$ é tame, o conjunto

$$
X:=\left\{\left\{z_{k}\right\} \subseteq \mathbb{C}^{n} \text { tal que a Condição 2.1 é satisfeita }\right\} \text { é vazio. }
$$

Logo, $f \in \mathcal{Q T}$.

Definição 2.6 Seja $f: \mathbb{C}^{n} \rightarrow \mathbb{C}$ uma função polinomial. Definimos o conjunto de Milnor de $f$ por:

$$
M(f):=\left\{z=\left(z_{1}, \cdots, z_{n}\right) \in \mathbb{C}^{n}: \bar{\nabla} f(z)=\lambda z ; \lambda \in \mathbb{C}\right\}
$$

Observação 2.8 Segue diretamente da definição, que $\Sigma_{f} \subset M(f)$.

Definição 2.7 Seja $f: \mathbb{C}^{n} \rightarrow \mathbb{C}$ uma função polinomial. Dizemos que $f$ é $M$-tame, se para toda sequência $\left\{z_{k}\right\} \subset M(f)$ tal que a Condição 2.1 é satisfeita, então $\lim _{k \rightarrow \infty}\left|f\left(z_{k}\right)\right|=\infty$. Denotaremos por $\mathcal{M T}$ a classe de polinômios M-tame.

Observação 2.9 Segue da observação 2.8 que, se $f \in \mathcal{M T}$, então $f$ tem somente singularidades isoladas. De fato, se tivermos uma curva $\varphi(t) \subset \Sigma_{f}$ tal que $\|\varphi(t)\| \rightarrow \infty$, isto implica $q u e \frac{d}{d t}(f(\varphi(t)))=\left\langle\frac{d}{d t}(\varphi(t)), \bar{\nabla} f(\varphi(t))\right\rangle=0$, portanto, $f(\varphi(t))=c \nrightarrow \infty$.

No que segue introduziremos algumas definições e resultados preliminares com base em [Pa], para mostrar que se $f \in \mathcal{Q T}$, então $f \in \mathcal{M T}$.

Definição 2.8 Dada $f: \mathbb{C}^{n} \rightarrow \mathbb{C}$ uma função polinomial e os seguintes conjuntos:

$$
\begin{gathered}
\widetilde{K}_{\infty}(f):=\left\{z_{0} \in \mathbb{C}: \exists\left\{z_{k}\right\} \subset \mathbb{C}^{n}, \text { com }\left\|z_{k}\right\| \rightarrow+\infty \text { tal que } f\left(z_{k}\right) \rightarrow z_{0} \text { e }\left\|\bar{\nabla} f\left(z_{k}\right)\right\| \rightarrow 0\right\} \\
e \\
K_{\infty}(f):=\left\{z_{0} \in \mathbb{C}: \exists\left\{z_{k}\right\} \subset \mathbb{C}^{n}, \text { com }\left\|z_{k}\right\| \rightarrow+\infty \text { tal que } f\left(z_{k}\right) \rightarrow z_{0} \text { e }\left\|z_{k}\right\| \cdot\left\|\bar{\nabla} f\left(z_{k}\right)\right\| \rightarrow 0\right\} .
\end{gathered}
$$


(a) Diremos que $f$ satisfaz a condição de Fedoryuk em $z_{0}$ ou que a fibra $f^{-1}\left(z_{0}\right)$ satisfaz a satisfaz a condição de Fedoryuk, se $z_{0} \notin \widetilde{K}_{\infty}(f)$, ou seja, se existe $\delta>0$ tal que para toda sequência $\left\|z_{k}\right\| \rightarrow+\infty$, com $f\left(z_{k}\right) \rightarrow z_{0}$ tivermos $\left\|\bar{\nabla} f\left(z_{k}\right)\right\| \geq \delta$.

(b) Diremos que $f$ satisfaz a condição de Malgrange em $z_{0}$ ou que a fibra $f^{-1}\left(z_{0}\right)$ satisfaz a condição de Malgrange, se $z_{0} \notin K_{\infty}(f)$, ou seja, se existir um $\delta>0$ tal que para toda sequência $\left\{z_{k}\right\}$, com $\left\|z_{k}\right\| \rightarrow+\infty$ e $f\left(z_{k}\right) \rightarrow z_{0}$, temos que

$$
\left\|z_{k}\right\| \cdot\left\|\bar{\nabla} f\left(z_{k}\right)\right\| \geq \delta
$$

(c) Diremos que a fibra $f^{-1}\left(z_{0}\right)$ satisfaz a condição de Malgrange fraca, se existirem $N \geq 1$ natural e um $\delta>0$ tal que, para toda sequência $\left\{z_{k}\right\}$, com $\left\|z_{k}\right\| \rightarrow+\infty$ e $f\left(z_{k}\right) \rightarrow z_{0}$, temos que

$$
\left\|z_{k}\right\|^{(N-1) / N} \cdot\left\|\bar{\nabla} f\left(z_{k}\right)\right\| \geq \delta .
$$

Definição 2.9 Diremos que uma função polinomial $f: \mathbb{C}^{n} \rightarrow \mathbb{C}$ satisfaz a condição de Fedoryuk (respectivamente, Malgrange fraca ou Malgrange), se $f$ satisfizer a condição de Fedoryuk (respectivamente, Malgrange fraca ou Malgrange) para todo $z_{0} \in \mathbb{C}$.

Decorre da definição que $K_{\infty}(f) \subseteq \widetilde{K}_{\infty}(f)$. No entanto, o exemplo a seguir mostra que, em geral, eles podem ser distintos.

Exemplo 2.10 Considere o polinômio homogêneo $f(x, y, z):=x^{2} y-x z^{2} \in \mathbb{C}[x, y, z], c \in \mathbb{C}^{*}$ e a curva

$$
\varphi:(0,1) \rightarrow \mathbb{C}^{3}, t \mapsto\left(t^{2}, \frac{c}{2} t^{-4}, \sqrt{c} t^{-1}\right)
$$

Assim, temos que

$$
\lim _{t \rightarrow 0}\|\varphi(t)\|=\infty, \lim _{t \rightarrow 0} f(\varphi(t))=-\frac{c}{2} \text { e } \lim _{t \rightarrow 0}\|\nabla f(\varphi(t))\|=0 .
$$

Consequentemente, $\mathbb{C}^{*} \subset \widetilde{K}_{\infty}(f)$. Agora considerando a curva $\beta(t)=\left(t^{4}, t^{-1}, 0\right)$, temos que $\{0\} \subset \widetilde{K}_{\infty}(f)$ e, consequentemente $\mathbb{C} \subset \widetilde{K}_{\infty}(f)$.

Por outro lado, tome $z_{0} \notin V$ e suponha que $f\left(z_{0}\right) \in K_{\infty}(f)$, ou seja, existe uma sequência $\left\{z_{k}\right\} \subset \mathbb{C}^{n},\left\|z_{k}\right\| \rightarrow+\infty$, tal que $f\left(z_{k}\right) \rightarrow f\left(z_{0}\right)$. Assim,

$$
0 \leq\left|3 \cdot f\left(z_{k}\right)\right|=\left|\left\langle z_{k}, \bar{\nabla} f\left(z_{k}\right)\right\rangle\right| \leq\left\|z_{k}\right\| \cdot\left\|\bar{\nabla} f\left(z_{k}\right)\right\| \rightarrow 0
$$

O que é uma contradição. Logo, $K_{\infty}(f) \varsubsetneqq \widetilde{K}_{\infty}(f)$.

Exemplo 2.11 O polinômio $f(x, y)=x-x^{2} y \in \mathbb{C}[x, y]$ não satisfaz a condição de Malgrange em $0 \in \mathbb{C}$. De fato, temos que

$$
\nabla f(x, y)=\left(1-2 x y,-x^{2}\right)
$$

e sendo $\alpha(t)=\left(t, \frac{1}{2 t}\right)$, temos que

$$
\nabla f(\alpha(t))=\left(0,-t^{2}\right)
$$


Dai, $\lim _{t \rightarrow 0}\|\alpha(t)\|=\infty, f(\alpha(t))=\frac{t}{2} e \lim _{t \rightarrow 0} \nabla f(\alpha(t))=0$. Mas,

$$
\begin{aligned}
\|\alpha(t)\|^{2} \cdot\|\nabla f(\alpha(t))\|^{2} & =\left(|t|^{2}+\frac{1}{4|t|^{2}}\right) \cdot|t|^{4} \\
& =\left(\frac{2|t|^{4}+1}{4}\right) \cdot|t|^{2} .
\end{aligned}
$$

Assim,

$$
\lim _{t \rightarrow 0}\|\alpha(t)\|^{2} \cdot\|\nabla f(\alpha(t))\|^{2}=\lim _{t \rightarrow 0}\left(\frac{2|t|^{4}+1}{4}\right) \cdot|t|^{2}=0 .
$$

E, portanto, $f(x, y)=x-x^{2} y$ não satisfaz a condição de Malgrange.

Proposição 2.12 Se $f: \mathbb{C}^{n} \rightarrow \mathbb{C}$ é uma função polinomial. Então, a condição de Fedoryuk implica a condição de Malgrange fraca, que por sua vez implica a condição de Malgrange.

DemonstraÇÃo: De fato, como $\frac{N-1}{N}=1-\frac{1}{N}<1$, para $z$ tal que $\|z\| \gg 1$, segue que $\|z\|^{(N-1) / N} \geq 1$. Assim, se existe $\delta>0$ tal que $\|\bar{\nabla} f(z)\|>\delta$, segue que $\|z\|^{(N-1) / N}$. $\|\bar{\nabla} f(z)\| \geq \delta$. Logo, segue a primeira implicação. Além disso, como $\|z\| \geq\|z\|^{(N-1) / N}$, segue que $\|z\| \cdot\|\bar{\nabla} f(z)\| \geq\|z\|^{(N-1) / N} \cdot\|\bar{\nabla} f(z)\| \geq \delta$. Portanto, segue a segunda implicação.

Lema 2.13 Se $f$ é quasitame, então $f$ satisfaz a condição de Malgrange.

DemonstraÇÃo: Com efeito, vamos provar usando a contra-positiva. Suponha que $f$ não satisfaz a condição de Malgrange em algum $z_{0} \in \mathbb{C}$, isto é, existe uma sequência $\left\{z_{k}\right\} \subseteq \mathbb{C}^{n}$ tal que

$$
\lim _{k \rightarrow \infty}\left\|z_{k}\right\|=\infty, \lim _{k \rightarrow \infty} f\left(z_{k}\right)=z_{0} \text { e } \lim _{k \rightarrow \infty}\left\|z_{k}\right\| \cdot\left\|\bar{\nabla} f\left(z_{k}\right)\right\|=0
$$

Logo,

$$
\begin{aligned}
\left|f\left(z_{k}\right)-\left\langle z_{k}, \bar{\nabla} f\left(z_{k}\right)\right\rangle\right| & \leq\left|f\left(z_{k}\right)\right|+\left|\left\langle z_{k}, \bar{\nabla} f\left(z_{k}\right)\right\rangle\right| \\
& \leq\left|f\left(z_{k}\right)\right|+\left\|z_{k}\right\| \cdot\left\|\bar{\nabla} f\left(z_{k}\right)\right\| \rightarrow\left|z_{0}\right| \not \rightarrow \infty
\end{aligned}
$$

Portanto, $f$ não é quasitame.

Proposição 2.14 Para $n \geq 2$, seja $f: \mathbb{C}^{n} \rightarrow \mathbb{C}$ uma função polinomial. Se $f$ satisfaz as condição de Malgrange, então $f$ é M-tame. 
DemonstraÇÃo: Vamos fazer a prova por redução ao absurdo. Assim, temos uma curva $p(t) \subset M(f)$ tal que $\|p(t)\| \rightarrow \infty$ e $|f(p(t))| \rightarrow z_{0}$ para algum $z_{0} \in \mathbb{C}$. Agora, consideremos as seguintes expansões:

$$
p(t):=a_{0} t^{\alpha}+a_{1} t^{\alpha+1}+\cdots
$$

em que cada $a_{i} \in \mathbb{C}^{n}$ para cada $i \geq 0, a_{0} \neq 0$ e $\alpha<0$,

$$
f(p(t)):=b_{0} t^{\beta}+b_{1} t^{\beta+1}+\cdots
$$

em que cada $b_{j} \in \mathbb{C}$ para cada $j \geq 0, b_{0} \neq 0$, por $(2.8)$ e $(2.9)$ temos que $f(p(t)) \not \equiv 0$ e $\beta \geq 0$, e

$$
\bar{\nabla}(f(p(t))):=c_{0} t^{\gamma}+c_{1} t^{\gamma+1}+\cdots
$$

em que cada $c_{l} \in \mathbb{C}^{n}$ para cada $l \geq 0, c_{0} \neq 0$, pois $\bar{\nabla}(f(p(t))) \not \equiv 0$ pela condição de Malgrange. Além disso, podemos escrever:

$$
\lambda(t):=\lambda_{0} t^{\sigma}+\lambda_{1} t^{\sigma+1}+\cdots
$$

em que cada $\lambda_{r} \in \mathbb{C}$ para cada $r \geq 0, \lambda_{0} \neq 0$.

Assim,

$$
\begin{aligned}
\bar{\nabla} f(p(t)) & =\lambda(t) \cdot p(t) \\
c_{0} t^{\gamma}+c_{1} t^{\gamma+1}+\cdots & =\left(\lambda_{0} t^{\sigma}+\lambda_{1} t^{\sigma+1}+\cdots\right) \cdot\left(a_{0} t^{\alpha}+a_{1} t^{\alpha+1}+\cdots\right) \\
c_{0} t^{\gamma}+c_{1} t^{\gamma+1}+\cdots & =\lambda_{0} \cdot a_{0} t^{\sigma+\alpha}+\cdots
\end{aligned}
$$

Desde que $a_{0}, c_{0}, \lambda_{0} \neq 0$, temos que

$$
\gamma=\sigma+\alpha
$$

Da identidade

$$
\begin{aligned}
\frac{d}{d t}(f(p(t)) & =\left\langle\frac{d p(t)}{d t}, \bar{\nabla} f(p(t))\right\rangle \\
\frac{d}{d t}(f(p(t)) & =\left\langle\frac{d p(t)}{d t}, \lambda(t) p(t)\right\rangle \\
\beta b_{0} t^{\beta-1}+(\beta+1) b_{1} t^{\beta}+\cdots & =\left\langle\alpha a_{0} t^{\alpha-1}+\cdots, \lambda_{0} a_{0} t^{\sigma+\alpha}+\cdots\right\rangle \\
\beta b_{0} t^{\beta-1}+(\beta+1) b_{1} t^{\beta}+\cdots & =\alpha \bar{\lambda}_{0}\left\|a_{0}\right\|^{2} t^{2 \alpha-1+\sigma}+\cdots
\end{aligned}
$$

Como $\alpha \bar{\lambda}_{0} \cdot\left\|a_{0}\right\|^{2} \neq 0$, segue que

$$
2 \alpha+\sigma \geq \beta \geq 0
$$

Das Equações (2.6) e (2.10), temos que

$$
\alpha+\gamma \geq \beta \geq 0
$$


Por outro lado, como $f$ satisfaz a condição de Malgrange, segue que

$$
\|p(t)\|^{2} \cdot\|\bar{\nabla} f(p(t))\|^{2} \geq \delta^{2}
$$

o que implica que

$$
\left\|a_{0}\right\|^{2} \cdot\left\|c_{0}\right\|^{2} t^{2(\alpha+\gamma)}+\cdots \geq \delta^{2}>0
$$

Assim,

$$
\alpha+\gamma \leq 0
$$

Das Equações (2.11) e (2.12), temos que

$$
\alpha+\gamma=\beta=0
$$

Por (2.8) segue que $f(p(t)) \not \equiv b_{0}$. Podemos reescrever (2.5) da seguinte forma

$$
f(p(t)):=b_{0}+b_{1} t^{\beta_{1}}+\cdots
$$

com $b_{1} \neq 0$ e $\beta_{1}>0$. Além disso, temos que

$$
b_{1} \beta_{1} t^{\beta_{1}-1}+\cdots=\alpha \bar{\lambda}_{0}\left\|a_{0}\right\|^{2} t^{2 \alpha-1+\sigma}+\cdots
$$

Portanto, $\beta_{1}=2 \alpha+\sigma$ e por (2.7) segue que $\beta_{1}=\gamma+\alpha$, o que é uma contradição por (2.13).

\subsection{Polinômios Semitame}

A seguir definiremos os polinômios semitame introduzidos por Némethi e Zaharia [NZ1]. Para tanto, consideraremos uma função polinomial $f: \mathbb{C}^{n} \rightarrow \mathbb{C}$ e uma sequência $\left\{z_{k}\right\} \subseteq$ $M(f)$ satisfazendo a Condição 2.1 - página 22.

Definição 2.10 Dada $f$ como acima. Diremos que $f$ é semitame, se

$$
\lim _{k \rightarrow \infty} f\left(z_{k}\right) \text { for finito, então } \lim _{k \rightarrow \infty} f\left(z_{k}\right)=0 .
$$

Denotaremos por $\mathcal{S T}$ o conjunto dos polinômios semitame.

Equivalentemente, diremos que $f$ é semitame se dado

$$
S T_{f}:=\left\{c \in \mathbb{C}:\left\{z_{k}\right\} \subset M(f),\left\|z_{k}\right\| \rightarrow+\infty \text { e } \bar{\nabla} f\left(z_{k}\right) \rightarrow 0 ; f\left(z_{k}\right) \rightarrow c\right\}
$$

tivermos que $S T_{f} \subseteq\{0\}$. 
Observação 2.15 Se $f \in \mathcal{M T}$, então $f \in \mathcal{S T}$. De fato, suponha que $f \notin \mathcal{S T}$, por definição, teríamos que existe uma sequência $\left\{z_{k}\right\} \subseteq M(f)$ tal que

$$
\lim _{k \rightarrow \infty}\left\|z_{k}\right\|=+\infty \text { e } \lim _{k \rightarrow \infty} \bar{\nabla} f\left(z_{k}\right)=0
$$

então, $\lim _{k \rightarrow \infty} f\left(z_{k}\right)=z_{0} \not \rightarrow 0$. Logo, $f \notin \mathcal{M T}$.

A Proposição a seguir pode ser encontrada em [NZ1, pg. 329], a qual manteremos o mesmo enunciado e prova.

Proposição 2.16 Se $f \in \mathcal{N N}_{0}$, então $f \in \mathcal{S T}$.

DemonstraÇÃo: Seja $f \in \mathcal{N N}_{0}$ e $p(t) \in M(f)$ uma curva analítica que satisfaz

$$
\lim _{t \rightarrow 0}\|p(t)\|=\infty, \lim _{t \rightarrow 0} \bar{\nabla} f(p(t))=0 .
$$

Podemos supor que

$$
\begin{array}{r}
p(t)=\left(z_{1}^{\circ} t^{a_{1}}+z_{1}^{1} t^{a_{1}+1}+\cdots,\right. \\
\left., z_{k}^{\circ} t^{a_{k}}+z_{k}^{1} t^{a_{k}+1}+\cdots, 0, \cdots, 0\right) \\
\\
\operatorname{com} z_{1}^{\circ} \neq 0, \cdots, z_{k}^{\circ} \neq 0 \text { e } a_{1}<0
\end{array}
$$

Seja $\varrho(t)$ tal que $\bar{\nabla} f(p(t))=\varrho(t) \cdot p(t)$. Quando $\varrho(t) \equiv 0$, obtemos de

$$
\frac{d f(p(t))}{d t}=\left\langle\frac{d p(t)}{d t}, \bar{\nabla} f(p(t))\right\rangle
$$

que $f(p(t))$ é uma constante, igual ao valor crítico, que deve ser zero, desde que $f \in \mathcal{N N}_{0}$. Se $\varrho(t) \not \equiv 0$, então $\varrho(t):=\varrho_{0} t^{\delta}+\varrho_{1} t^{\delta+1}+\cdots$, com $\varrho_{0} \neq 0$, e $\frac{\partial f}{\partial z_{1}}(p(t)) \not \equiv 0$. Logo, identificando $\mathbb{R}^{k}$ com $\left\{x \in \mathbb{R}^{n}: x_{k+1}=\cdots=x_{n}=0\right\}$, temos $\operatorname{supp}(f) \cap \mathbb{R}^{k} \neq \emptyset$. Seja $d$ o valor mínimo da função $l_{a}: \overline{\operatorname{supp}}(f) \cap \mathbb{R}^{k} \rightarrow \mathbb{R}, l_{a}(x)=\sum_{j=1}^{k} a_{j} x_{j}$, e seja $\Delta$ a única face de $\overline{\operatorname{supp}}(f) \cap \mathbb{R}^{k}$, em que $l_{a}$ toma este valor. Então

$$
f(p(t))=f_{\Delta}\left(z^{\circ}\right) t^{d}+\cdots
$$

e se $d>0$ não existe nada a provar. Se $d=0$ e $a_{j} \leq 0$ para cada $j=1, \cdots, k$, então $f\left(z_{1}, \cdots, z_{k}, 0, \cdots, 0\right)$ não depende de $z_{1}$. Portanto, $\frac{\partial f}{\partial z_{1}}(p(t)) \equiv 0$, uma contradição.

Se $d=0$ e existe $j \in\{1, \cdots, k\}$ com $a_{j}>0$, as condições $(a)$ e (b) da definição de face ruim são satisfeitas, quando temos

$$
H=\left\{x \in \mathbb{R}^{n}: \sum_{j=1}^{k} a_{j} x_{j}=0\right\}
$$

Como $f \in \mathcal{N N}_{0}$, segue que $(c)$ não é satisfeita. Portanto, $\Delta$ é uma face de $\widetilde{\Gamma}(f)$. Pela condição de não-degenericidade da face $\Delta$, existe $q \in\{1, \cdots, k\}$ tal que

$$
\frac{\partial f_{\Delta}}{\partial z_{q}}\left(z^{\circ}\right) \neq 0
$$


Além disso, como para todo $j \in\{1, \cdots, k\}$, temos que $\frac{\partial f}{\partial z_{j}}(p(t))=\frac{\partial f_{\Delta}}{\partial z_{j}}(p(t)) t^{d-a_{j}}+\cdots$ tende a zero quando $t \rightarrow 0$, assim $d-a_{q}>0$, o que significa que $a_{q}<0$. Seja $I=\left\{i: a_{i}=\right.$ $\left.a_{q}\right\}$. A condição $p(t) \in M(f)$ implica que $a_{q}=\min \left\{a_{1}, \cdots, a_{q}\right\}$ e para $j \in I, d-a_{j}=\delta+a_{j}$ $\mathrm{e}$

$$
\bar{\varrho}_{0} \cdot \overline{z_{j}^{\circ}}=\frac{\partial f_{\Delta}}{\partial z_{j}}\left(z^{\circ}\right) \neq 0
$$

Também, para $j \in I$ temos $d-a_{j}<\delta+a_{j}$, portanto

$$
\frac{\partial f_{\Delta}}{\partial z_{j}}\left(z^{\circ}\right)=0
$$

Logo a relação de Euler torna-se para $f_{\Delta}$ :

$$
\sum_{j \in I} a_{j} z_{j}^{\circ} \frac{\partial f_{\Delta}}{\partial z_{j}}\left(z^{\circ}\right)=d \cdot f_{\Delta}\left(z^{\circ}\right)
$$

e isto implica que $\bar{\varrho}_{0} \cdot \sum_{j \in I}\left|z_{j}^{\circ}\right|^{2}=0$, o que é uma contradição.

Quando $d<0$ e $f_{\Delta}\left(z^{\circ}\right) \neq 0$ não existe nada a provar. Se $d<0$ e $f_{\Delta}\left(z^{\circ}\right)=0$ obtemos uma relação como (2.14) e ocorre novamente a igualdade absurda $\bar{\varrho}_{0} \cdot \sum_{j \in I}\left|z_{j}^{\circ}\right|^{2}=0$.

Observação 2.17 (a) O exemplo 2.2 - página 21 mostra que a recíproca da observação 2.15 não é verdadeira em geral. De fato, $f \in \mathcal{N N}_{0}$ e portanto $f \in \mathcal{S T}$. No entanto, $f \notin \mathcal{M T}$, pois a reta complexa $\{(x, 0): x \in \mathbb{C}\} \subset \Sigma_{f}$.

(b) Diante do exposto anteriormente temos o seguinte diagrama de inclusões:

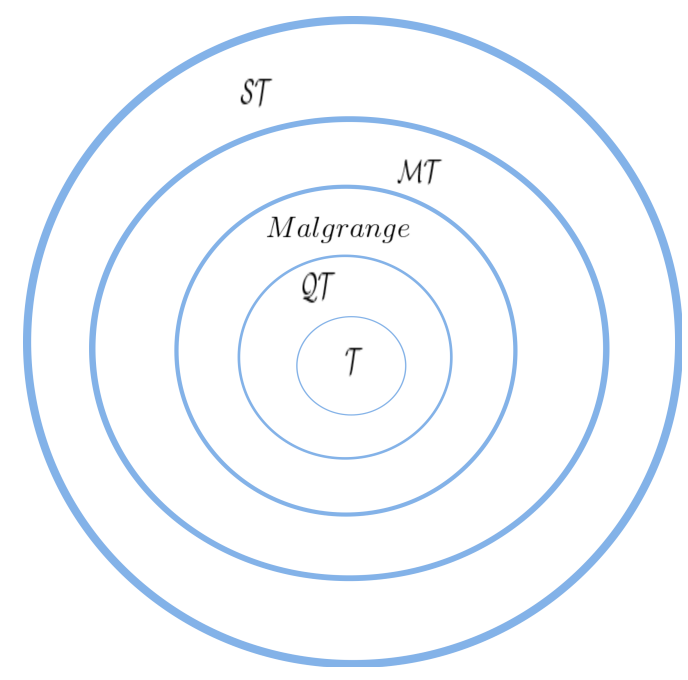

Além disso, dada $f: \mathbb{C}^{n} \rightarrow \mathbb{C}$ como anteriormente, se $n=2$ então segue de [Ti] que as condições Quasitame, Malgrange e M-tame coincidem. E, se $n \geq 3$, decorre de [NZ1, pg. 330] e de [PZ, pg. 271] que todas as inclusões são estritas. 


\section{Capítulo 3}

\section{Fibrações em Esferas}

\section{Sumário}

3.1 O Teorema de fibração para polinômios Semitame $\ldots . . . .31$

3.2 Decomposição open book . . . . . . . . . . . . 37

Em [BP1, BP2] A. Bodin e A. Pichon adaptaram o conceito de semitame para funções meromorfas com o objetivo de estender a fibração de Milnor local para o ambiente global. Com o mesmo objetivo e utilizando como ferramenta principal o poliedro de Newton no infinito, M. Oka [Ok] apresentou um estudo dos polinômios mistos, que é uma classe especial de polinômios que estão na fronteira entre as funções complexas holomorfas e as aplicações analíticas reais. Também em [A-C1, A-C2] A. Aguilar-Cabrera fez um estudo dos germes de polinômios mistos do tipo $f \bar{g}+z^{n}:\left(\mathbb{C}^{3}, 0\right) \rightarrow(\mathbb{C}, 0)$, em que $f, g$ são funções holomorfas, apresentando uma família de aplicações analíticas reais cuja fibração de Milnor associada não é proveniente de uma função holomorfa.

Na próxima seção veremos que a classe de polinômios semitame apresentada no capítulo anterior satisfaz um teorema de fibração em grandes esferas.

\subsection{O Teorema de fibração para polinômios Semitame}

No caso complexo global, dada uma função polinomial não constante $f: \mathbb{C}^{n} \rightarrow \mathbb{C}$ é um problema interessante decidir quando a projeção

$$
\phi:=\frac{f}{\|f\|}: S_{R}^{2 n-1} \backslash K_{R} \rightarrow S^{1}
$$

é uma fibração suave para todo $R \gg 1$, em que $K_{R}:=V \cap S_{R}^{2 n-1}$ é o link da singularidade no infinito, em que $V:=f^{-1}(0)$.

Definição 3.1 Diremos que uma função polinomial não constante $f: \mathbb{C}^{n} \rightarrow \mathbb{C}$ admite uma fibração de Milnor no infinito, se existir a fibração (3.1) para todo $R \gg 1$. Neste caso, diremos que as fibras $F_{\theta}:=\phi^{-1}\left(e^{i \theta}\right)$ são as fibras de Milnor no infinito. 
Diferentemente do que ocorre no caso holomorfo local, em geral, nem sempre a fibração de Milnor no infinito existe. O principal objetivo desta seção é apresentar o Teorema de fibração para polinômios semitame (Teorema 3.1 - página 32), conforme [NZ1], como segue.

Teorema 3.1 Se $f \in \mathcal{S T}$, então para $R_{0} \gg 1$ a aplicação (3.1) é uma fibração suave localmente trivial.

Na prova serão necessários alguns lemas, os quais seguiremos enunciado e a ideia da prova conforme [Mil1, NZ1]. O primeiro deles é o seguinte:

Lema 3.2 Sejam $f \in \mathcal{S} \mathcal{T}, p:(0, \epsilon) \rightarrow \mathbb{C}^{n}$ uma curva analítica tal que $\lim _{t \rightarrow 0}\|p(t)\|=\infty$ o número $f(p(t))$ é diferente de zero e o vetor $\bar{\nabla} \log f(p(t))$ é um múltiplo complexo de $\lambda(t) \cdot p(t)$. Então o argumento do número complexo $\lambda(t)$ tende a zero ou $\pi$ quando $t \rightarrow 0$.

DemonstraÇÃo: Considere as seguintes expansões:

$$
p(t):=a_{0} t^{\alpha}+a_{1} t^{\alpha+1}+\cdots
$$

em que cada $a_{i} \in \mathbb{C}^{n}$ para cada $i \geq 0, a_{0} \neq 0$ e $\alpha<0$,

$$
f(p(t)):=b_{0} t^{\beta}+b_{1} t^{\beta+1}+\cdots
$$

em que cada $b_{j} \in \mathbb{C}$ para cada $j \geq 0, b_{0} \neq 0$ e

$$
\bar{\nabla} f(p(t)):=c_{0} t^{\gamma}+c_{1} t^{\gamma+1}+\cdots
$$

em que cada $c_{l} \in \mathbb{C}^{n}$ para cada $l \geq 0$.

Se $\bar{\nabla} f(p(t)) \equiv 0$, segue da identidade

$$
\frac{d}{d t}(f(p(t)))=\left\langle\frac{d p(t)}{d t}, \bar{\nabla} f(p(t))\right\rangle
$$

que $f(p(t))$ é constante não nulo, e isto contradiz a hipótese que $f \in \mathcal{S T}$.

Por outro lado, como $\bar{\nabla} \log f(p(t))$ é um múltiplo complexo de $\lambda(t) \cdot p(t)$, segue que

$$
\bar{\nabla} f(p(t))=\lambda(t) \cdot p(t) \cdot \overline{f(p(t))}
$$

com isto temos que $p(t) \in M(f), c_{0} \neq 0$ e

$$
c_{0} t^{\gamma}+c_{1} t^{\gamma+1}+\cdots=\lambda(t) \cdot\left[\left(a_{0} \overline{b_{0}}\right) t^{\alpha+\beta}+\cdots\right] .
$$

Desta forma, decorre que

$$
\lambda(t)=\lambda_{0} t^{\gamma-\alpha-\beta}+\lambda_{1} t^{\gamma-\alpha-\beta+1}+\lambda_{2} t^{\gamma-\alpha-\beta+2}+\cdots
$$

com $\lambda_{0} \neq 0$ e $c_{0}=\lambda_{0} a_{0} \overline{b_{0}}$. Além disso, das identidades (3.2) e (3.3), temos que

$$
\beta b_{0} t^{\beta-1}+(\beta+1) b_{1} t^{\beta}+\cdots=\alpha\left\|a_{0}\right\|^{2} \bar{\lambda}_{0} b_{0} t^{\alpha-1+\gamma}+\cdots
$$

Se $\beta=0$, então $\alpha+\gamma \geq 1$, portanto $\gamma>0$ e $f(p(t)) \rightarrow b_{0} \in \mathbb{C}^{*}$, o qual é uma contradição com o fato que $f \in \mathcal{S} \mathcal{T}$. Logo, $\beta \neq 0$ e, consequentemente, $\beta=\alpha\left\|a_{0}\right\|^{2} \overline{\lambda_{0}}$ e $\lambda_{0}$ é um número real. Portanto,

$$
\lim _{t \rightarrow 0} \frac{\lambda(t)}{\|\overline{\lambda(t)}\|}=\frac{\lambda_{0}}{\left\|\overline{\lambda_{0}}\right\|}= \pm 1 \text {, isto é, } \lim _{t \rightarrow 0} \arg (\lambda(t))=0
$$


Lema 3.3 Seja $f \in \mathcal{S T}$. Se existe um número real $R_{0} \gg 1$ tal que para todo $z \in \mathbb{C}^{n} \backslash V$, $\|z\| \geq R_{0} \operatorname{com} \bar{\nabla} \log f(z)=\lambda z, \lambda \neq 0$, então $|\operatorname{Im} \lambda|<\mid$ Re $\lambda \mid$.

Demonstração: Seja $p:(0, \epsilon) \rightarrow \mathbb{C}^{n}$ uma curva analítica tal que $p(t) \in \mathbb{C}^{n} \backslash V$, assim o número $f(p(t))$ é diferente de zero. Como $\bar{\nabla} \log f(p(t))=\lambda(t) \cdot p(t)$, segue que $p(t) \in M(f)$. Portanto, pelo Lema anterior, temos que

$$
\lim _{t \rightarrow 0} \arg (\lambda(t))=0 \text { e }|\operatorname{Im} \lambda(t)|<|\operatorname{Re} \lambda(t)|
$$

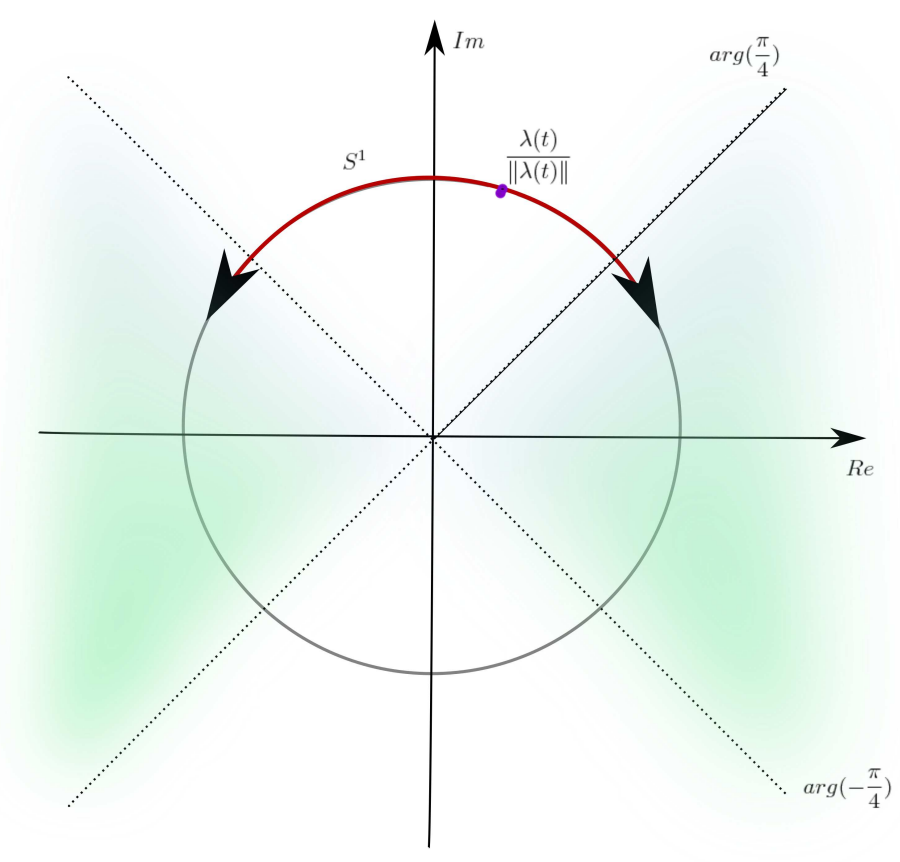

No que segue, mostraremos que estudar os pontos críticos de $\phi(z)$ é equivalente a estudar os pontos críticos de $\theta(z)$, em que $\theta(z)$ é o argumento do número complexo $\lambda(t)$.

Lema 3.4 Seja $f: \mathbb{C}^{n} \rightarrow \mathbb{C}$ uma função polinomial. Então, $z \in S_{R} \backslash K_{R}$ é um ponto crítico de $\phi$ se, e somente se, existe um $\lambda \in \mathbb{R}$ tal que $i \bar{\nabla} \log f(z)=i \frac{\bar{\nabla} f(z)}{\overline{f(z)}}=\lambda \cdot z$.

DemonstraÇão: Inicialmente, observe que para $z \in S_{R} \backslash K_{R}$, isto é, $f(z) \neq 0$, podemos escrever

$$
\phi(z):=\frac{f(z)}{|f(z)|}=e^{i \theta(z)} .
$$

Como, localmente o logaritmo complexo está bem definido, temos que:

$$
\begin{aligned}
\log \left(e^{i \theta(z)}\right) & =\log \left(\frac{f(z)}{|f(z)|}\right) \\
i \theta(z) & =\log f(z)-\log |f(z)| \\
\theta(z) & =i \log |f(z)|-i \log f(z)
\end{aligned}
$$


Como $\theta(z)$ é real e $i \log |f(z)|$ é imaginário puro, temos que $\theta(z)=R e(-i \log f(z))$ e, portanto,

$$
\nabla \theta(z)=\nabla(\operatorname{Re}(-i \log f(z)))
$$

Agora, considere $z_{0} \in S_{R} \backslash K_{R}$ um ponto crítico de $\phi$ e uma curva diferenciável $\alpha$ : $(-\epsilon, \epsilon) \rightarrow S_{R} \backslash K_{R} \operatorname{com} \alpha(0)=z_{0}$ e $\alpha^{\prime}(0)=v \neq 0$. Assim, temos que

$$
0=\frac{d \phi}{d v}\left(z_{0}\right)=\left\langle v, \bar{\nabla} \phi\left(z_{0}\right)\right\rangle
$$

Por outro lado, $\phi(z)=\frac{f(z)}{\|f(z)\|}=e^{i \theta(z)} \Rightarrow \bar{\nabla} \phi(z)=-i \cdot e^{i \theta(z)} \cdot \nabla \theta(z)$. Por (3.4), segue que $\bar{\nabla} \phi(z)=-i \cdot e^{i \theta(z)} \cdot \nabla(\operatorname{Re}(-i \log f(z)))$.

Daí, substituindo $\bar{\nabla} \phi(z)$ em (3.5), temos:

$$
0=\frac{d \phi}{d v}\left(z_{0}\right)=\left\langle v,-i e^{i \theta\left(z_{0}\right)} \nabla\left(\operatorname{Re}\left(-i \log f\left(z_{0}\right)\right)\right)\right\rangle .
$$

Donde segue que $\operatorname{Re}\left(\left\langle v, i \bar{\nabla}\left(\log f\left(z_{0}\right)\right)\right)\right\rangle=0$. E, portanto, $i \bar{\nabla}\left(\log f\left(z_{0}\right)\right)=\lambda z_{0}$.

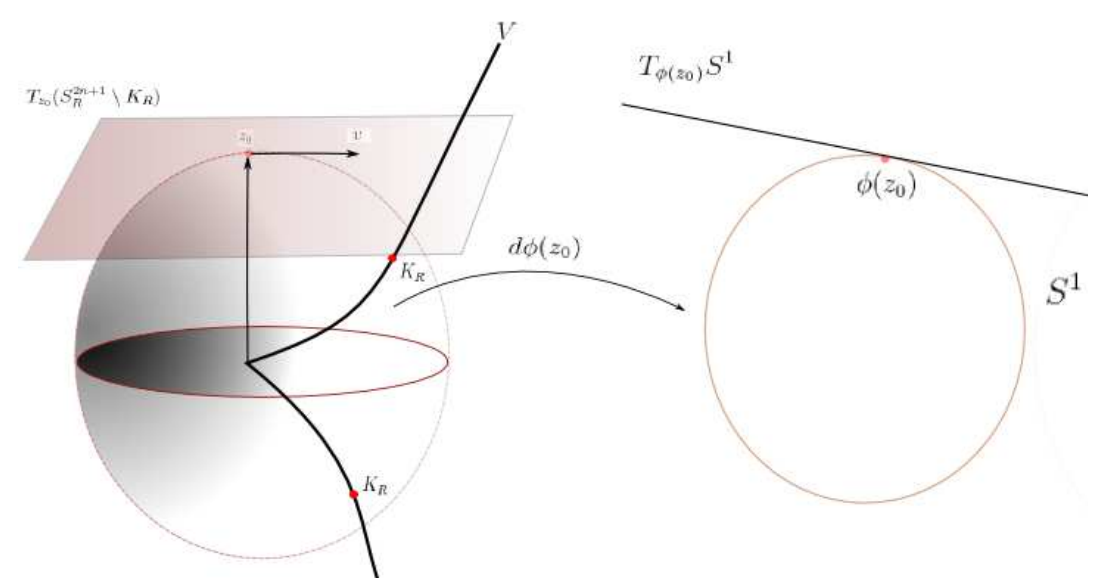

Observação 3.5 Decorre do Lema anterior e da observação 2.8 - página 24 que se $f$ : $\mathbb{C}^{n} \rightarrow \mathbb{C}$ é uma função polinomial com singularidade não isolada em $\mathbb{C}^{n} \backslash V$, então $\phi$ não é submersão. O que não pode ocorrer se $f \in \mathcal{S T}$.

Corolário 3.6 Seja $f \in \mathcal{S T}$. Então, para todo $R \gg 1$ a aplicação

$$
\phi:=\frac{f}{\|f\|}: S_{R}^{2 n-1} \backslash K_{R} \rightarrow S^{1}
$$

é uma submersão suave.

DemonstraÇÃo: Decorre do Lema 3.3 - página 33 , que $\bar{\nabla} \log f(z)$ e $z$ são $\mathbb{C}$-linearmente independentes ou, se $\bar{\nabla} \log f(z)=\lambda z$, então $|\operatorname{Im} \lambda|<|\operatorname{Re} \lambda|$. No entanto, esta desigualdade não permite que $\lambda$ seja imaginário puro. Pelo Lema anterior segue o resultado. 
Lema 3.7 Seja $f \in \mathcal{S T}$ e $R_{0} \gg 1$ como no Lema 3.3. Então para cada $R \geq R_{0}$ existe um campo suave de vetores tangente $v(z)$ sobre $S_{R} \backslash K_{R}$ tal que para cada $z \in S_{R} \backslash K_{R}$ o produto interno complexo $\langle v(z), i \bar{\nabla} \log f(z)\rangle$ é diferente de zero e tem argumento menor que $\pi / 4$ em valor absoluto.

DemonstraçÃo: A prova é similar a prova do [Mil1, Lema 4.6, pg. 40].

Lema 3.8 Dado qualquer $z \in S_{R} \backslash K_{R}$, existe um único caminho suave $\alpha: \mathbb{R} \rightarrow S_{R} \backslash K_{R}$ tal que $\frac{d \alpha(t)}{d t}=w(\alpha(t))$ com condição inicial $\alpha(0)=z$, em que $w(z)=\frac{v(z)}{\operatorname{Re}\langle v(z), i \bar{\nabla} \log f(z)\rangle}$.

Demonstração: Pelo Teorema de existência e unicidade de solução de equação diferencial, tal solução $z=\alpha(t)$ existe localmente e esta solução pode ser estendida a um intervalo aberto maximal da reta.

Como $S_{R} \backslash K_{R}$ não é compacto, é suficiente mostrar que não existe $t_{0} \in \mathbb{R}$ tal que $\alpha(t) \nrightarrow \rightarrow K_{R}$ quando $t \rightarrow t_{0}$. Ou seja, que $f(\alpha(t)) \not \rightarrow 0$, quando $t \rightarrow t_{0}$, ou equivalentemente que, $\operatorname{Re}(\log f(\alpha(t))) \not \rightarrow \infty$ quando $t \rightarrow t_{0}$. Para mostramos que isso não ocorre, vamos mostrar que a derivada de $\log f(\alpha(t))$ é limitada. Com efeito,

$$
\left|\frac{d}{d t}[\operatorname{Re} \log f(\alpha(t))]\right|=|\operatorname{Re}\langle w(\alpha(t)), \bar{\nabla} \log (f(\alpha(t)))\rangle|<1
$$

Observe que

$$
\begin{aligned}
\frac{d \theta(\alpha(t))}{d t} & =\operatorname{Re}\left\langle\frac{d \alpha(t)}{d t}, i \bar{\nabla} \log (f(\alpha(t)))\right\rangle \\
& =\operatorname{Re}\langle w(\alpha(t)), i \bar{\nabla} \log (f(\alpha(t)))\rangle \\
& =\operatorname{Re}\left\langle\frac{v(\alpha(t))}{\operatorname{Re}\langle v(\alpha(t)), i \bar{\nabla} \log (f(\alpha(t)))\rangle}, i \bar{\nabla} \log (f(\alpha(t)))\right\rangle \\
& =\frac{1}{\operatorname{Re}\langle v(\alpha(t)), i \bar{\nabla} \log (f(\alpha(t)))\rangle} \cdot \operatorname{Re}\langle v(\alpha(t)), i \bar{\nabla} \log (f(\alpha(t)))\rangle \\
& =1
\end{aligned}
$$

Segue que $\theta(\alpha(t))=t+\theta_{0}$, em que $\theta_{0}$ é uma constante.

Assim,

$$
\phi(\alpha(t)):=\frac{f(\alpha(t))}{|f(\alpha(t))|}=e^{i \theta(\alpha(t))}=e^{i\left(t+\theta_{0}\right)}=e^{i t} e^{i \theta_{0}}, \quad \forall t \in \mathbb{R}
$$

Portanto, $\phi$ é submersão sobrejetora suave. Para completar a prova do Teorema 3.1, resta mostrar a trivialidade local. Para isso considere $h_{t}: S_{R} \backslash K_{R} \rightarrow S_{R} \backslash K_{R}$ o difeomorfismo 
definido por $(z, t) \mapsto \alpha_{z}(t)$, em que $\alpha_{z}: \mathbb{R} \rightarrow S_{R} \backslash K_{R}$ é a única curva integral com $\alpha_{z}(0)=z$ para cada $t \in \mathbb{R}$. Consequentemente, se $z \in F_{\theta}$,

$$
\phi\left(h_{t}(z)\right)=\phi\left(\alpha_{z}(t)\right)=e^{i \theta\left(\alpha_{z}(t)\right)}=e^{i\left(t+\theta\left(\alpha_{z}(0)\right)\right)}=e^{i(t+\theta(z))}=e^{i(t+\theta)} .
$$

Portanto, para cada $t \in \mathbb{R}$ o difeomorfismo $h_{t}$ leva a fibra $F_{\theta}:=\phi^{-1}\left(e^{i \theta}\right)$ sobre a fibra $F_{\theta+t}$. Para mais detalhes dos argumentos básicos o leitor interessado pode consultar, por exemplo, [CS, pg. 30].

Com base no que foi discutido neste capítulo e no capítulo anterior, temos o seguinte diagrama de inclusões.

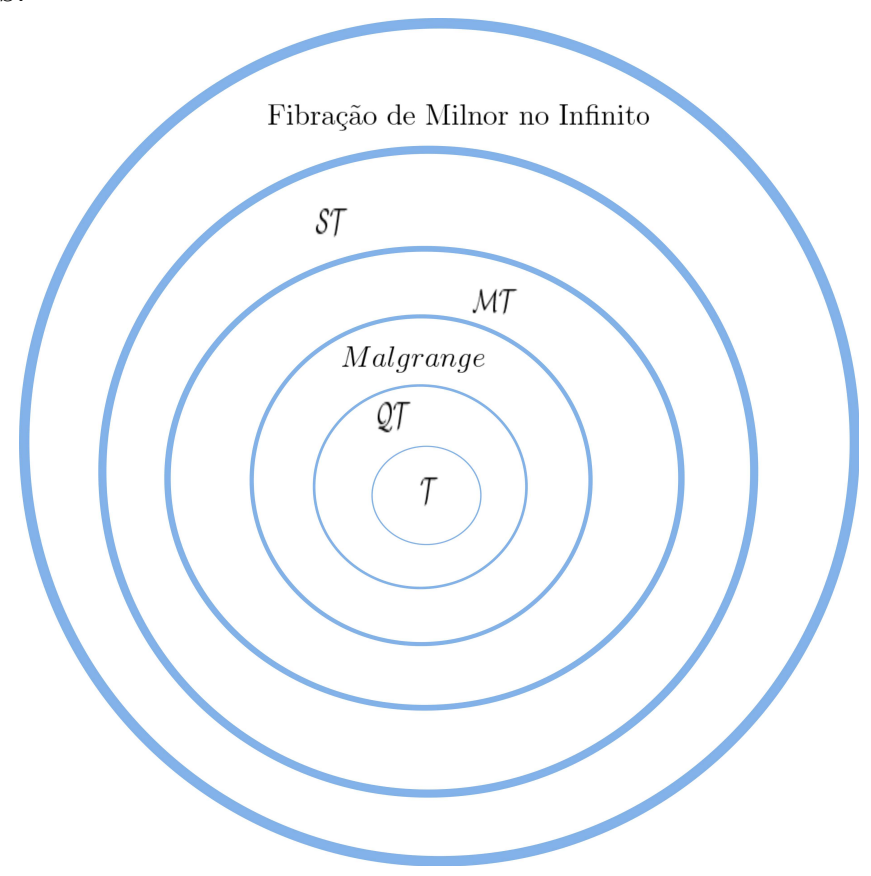

Uma pergunta natural é: a existência da fibração de Milnor no infinito para $f$, implica $f \in \mathcal{S T}$ ?

Nesta direção, Arnaud Bodin [Bo] provou a seguinte caracterização:

Teorema 3.9 Dada $f: \mathbb{C}^{2} \rightarrow \mathbb{C}$ uma função polinomial. Então, $f \in \mathcal{S T}$, se e somente se, $f$ admite fibração de Milnor no infinito.

Considerando $f: \mathbb{C}^{n} \rightarrow \mathbb{C}$ polinomial holomorfa, decorre das discussões anteriores que se $f$ admite fibração de Milnor no infinito, então $\phi$ é uma submersão para todo $R \gg 1$. Além disso, o conjunto singular, $\Sigma_{f /\|f\|}$, da projeção $\frac{f}{\|f\|}: \mathbb{C}^{n} \backslash V \rightarrow S^{1}$ é um conjunto finito de pontos. Pelo Lema 3.4 - página 33, temos que $\Sigma_{f} \backslash V \subset \Sigma_{f /\|f\|}$ e portanto $f$ admite um número finito de pontos críticos em $\mathbb{C}^{n} \backslash V$. Com isto, temos o seguinte diagrama mais geral. 


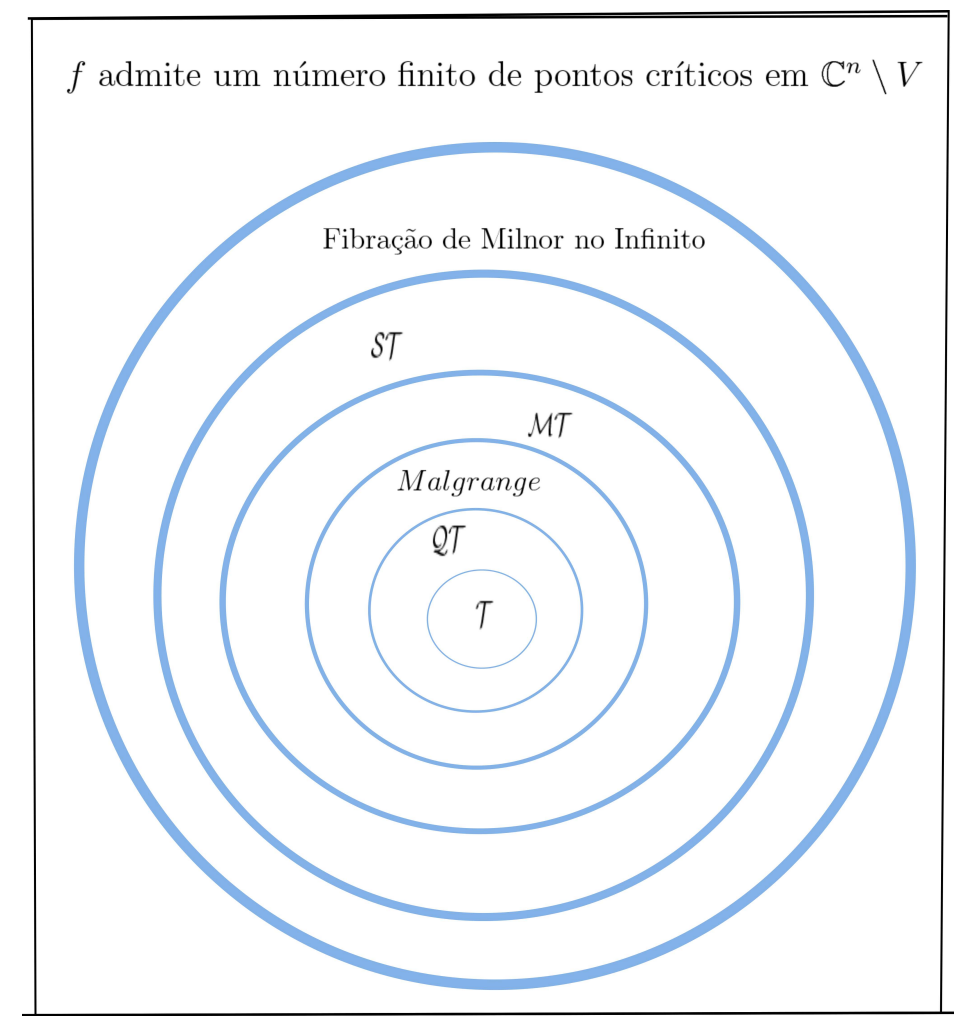

Para o caso $n>2$ a recíproca do Teorema 3.1 é ainda um problema em aberto. No entanto, uma recíproca parcial sob a condição Newton não-degenerada no infinito pode ser encontrada em [Ch, Teorema 4.1.3, pg. 32].

\subsection{Decomposição open book}

O conceito clássico de estrutura open book foi denominado por Winkelnkemper [Wi]. Este conceito aparece na literatura com vários outros nomes: fibered links, Neuwrith-stallings pairs etc (para mais detalhes, veja por exemplo [Lo]).

Uma classe importante de exemplos de estrutura estrutura open book pode ser encontrado em [Mil1] ao considerarmos um germe de função holomorfa $f:\left(\mathbb{C}^{n}, 0\right) \rightarrow(\mathbb{C}, 0)$ com singularidade isolada.

Nesta seção, nossas principais referências são os artigos [ACT, ACT1], no qual os autores utilizam uma generalização do conceito de decomposição open book para obter fibrações suaves localmente triviais sobre esferas de raios suficientemente grandes (Teorema 3.12 página 39).

A seguir apresentaremos algumas notações e definições mais gerais.

Sejam $F=\left(f_{1}, \cdots, f_{p}\right): \mathbb{R}^{n} \rightarrow \mathbb{R}^{p}, n>p \geq 2$ uma aplicação polinomial, $V:=F^{-1}(0)$, $F /\|F\|: \mathbb{R}^{n} \backslash V \rightarrow S^{p-1}$ e $\rho: \mathbb{R}^{n} \rightarrow \mathbb{R}, \rho(x):=\|x\|^{2}$. Considere os conjuntos $M(F):=\Sigma_{(F, \rho)}$ e $M(F /\|F\|):=\Sigma_{(F /\|F\|, \rho)}$, conjuntos de Milnor de $F$ e da projeção $F /\|F\|$, respectivamente.

Observação 3.10 (a) Segue das definições acima, que $M(F)$ é um conjunto algébrico 
fechado e que $M(F /\|F\|)$ é um conjunto semi-algébrico relativamente fechado.

(b) Decorre também das definições acima que $M(F /\|F\|) \subset M(F) \backslash V$, pois, se $x \notin V$ é tal que $\rho \pitchfork_{x} F$, então $\rho \pitchfork_{x} F /\|F\|$.

(c) Além disso, analisando o diagrama abaixo:

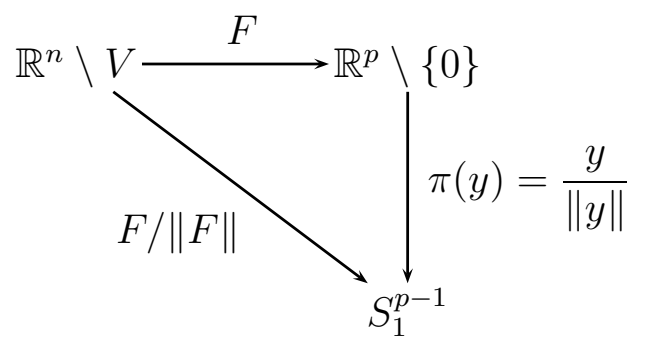

temos o seguinte diagrama de inclusões:

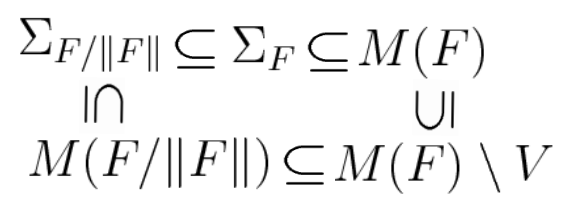

Definição 3.2 Seja $X \subset \mathbb{R}^{n}$ um conjunto semi-algébrico ilimitado. Diremos que $X$ tem dimensão $r$ no infinito, se existir um raio $R \gg 1$ e $B_{R}^{n}(0)$ tal que cada componente irredutivel de $X$ em $X \backslash\left(\Sigma(X) \cup B_{R}^{n}(0)\right)$ tem dimensão $r$. Neste caso, também diremos que a codimensão de $X$ no infinito é $n-r$ e denotaremos por $\operatorname{codim}_{\infty}(X)=n-r$.

Definição 3.3 Diremos que o par $(K, \theta)$ é uma estrutura open book superior com binding singular em uma variedade analítica $M$ de dimensão $m-1 \geq p \geq 2$, se $K \subset M$ é uma subvariedade real singular de codimensão p e $\theta: M \backslash K \rightarrow S_{1}^{p-1}$ é uma fibração suave localmente trivial, tal que, $K$ admite uma vizinhança $N$ para a qual $\theta_{\mid N \backslash K}$ é a composição

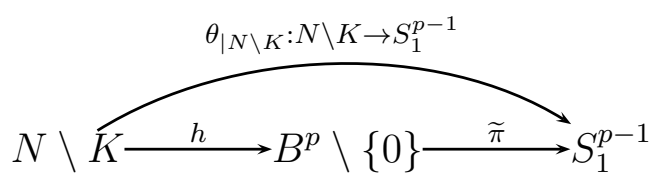

em que h é uma fibração suave localmente trivial e $\tilde{\pi}$ é a projeção radial. Diremos que a subvariedade singular $K$ é o binding $e$ que o (fecho das) fibras de $\theta$ são as páginas do open book.

Com as notações e definições acima em $[\mathrm{ACT}]$ os autores provaram o seguinte resultado.

Teorema 3.11 [ACT, Teorema 1.3, pg. 819] Seja $F:\left(\mathbb{R}^{n}, 0\right) \rightarrow\left(\mathbb{R}^{p}, 0\right)$ um germe de aplicação analítica, $n>p \geq 2$, tal que $\operatorname{codim}(V)=p$, em que $V=F^{-1}(0)$. Suponha que $\overline{M(F) \backslash V} \cap V \subseteq\{0\}$. Se $M(F /\|F\|)=\emptyset$, então $\left(K_{\epsilon}, F /\|F\|\right)$ é uma decomposição open book de $S_{R}^{n-1}$ com binding singular, independente (a menos de isotopia) do raio $\epsilon>0$ suficientemente pequeno. 
Em [ACT1] os autores provaram uma contraparte deste resultado no aspecto global, como segue.

Teorema 3.12 [ACT1, Teorema 3.2, pg. 4] Seja $F: \mathbb{R}^{n} \rightarrow \mathbb{R}^{p}$ uma aplicação polinomial real tal que $\operatorname{codim}_{\infty}(V)=p$. Além disso, assuma que $\overline{M(F) \backslash V} \cap V$ é limitado. Então são equivalentes:

(a) $\left(K_{R}, F /\|F\|\right)$ é uma decomposição open book de $S_{R}^{n-1}$ com binding singular, independente do raio $R \gg 1$, a menos de $C^{\infty}$ de isotopia.

(b) $M(F /\|F\|)$ é limitado.

Omitiremos a prova do Teorema acima, pois provaremos um resultado mais geral (Teorema 4.1 - página 42 ).

Observação 3.13 As hipóteses do Teorema 3.12 não excluem a possibilidade de ramos ilimitados no conjunto $\Sigma_{F} \cap V$.

Lema 3.14 Se $\Sigma_{F} \cap V$ é limitado, então $\overline{M(F) \backslash V} \cap V$ é limitado.

DemonstraÇÃo: Basta considerar $\rho: V \rightarrow \mathbb{R}$ e usar a aplicação 1.12 - página 10.

Corolário 3.15 Seja $F: \mathbb{R}^{n} \rightarrow \mathbb{R}^{p}$ uma aplicação polinomial real tal que $\operatorname{codim}_{\infty}(V)>0$ e seja $\Sigma_{F} \cap V$ limitado. Então $\left(K_{R}, F /\|F\|\right)$ é uma decomposição open book de $S_{R}^{n-1}$, independente do $R \gg 1$, a menos de isotopia, se, e somente se, $M(F /\|F\|)$ é limitado.

Observação 3.16 A hipótese que o conjunto $\Sigma_{F} \cap V$ é limitado é a mais geral sobre a qual $\left(K_{R}, F /\|F\|\right)$ pode dar origem a uma estrutura open book sobre $S_{R}^{n-1}$ com link não-singular.

Como vimos, no caso global de uma aplicação polinomial complexa $f: \mathbb{C}^{n} \rightarrow \mathbb{C}$, usando o método de Milnor para grandes esferas, em [NZ1] Némethi e Zaharia mostraram a existência da fibração de Milnor no infinito para $f \in \mathcal{S} \mathcal{T}$. O Corolário a seguir nos mostra como obter este resultado usando a técnica da decomposição open book no infinito.

Corolário 3.17 [ACT1, Teorema 3.6, pg. 5] Seja $f: \mathbb{C}^{n} \rightarrow \mathbb{C}, n \geq 2$, um polinômio complexo não-constante. Se $f \in \mathcal{S T}$, então, $\left(K_{R}, f /\|f\|\right)$ é uma decomposição open book de $S_{R}^{2 n-1}$ com link singular, independente do raio $R \gg 1$, a menos de $C^{\infty}$ isotopia.

Demonstração: De fato, claramente $\operatorname{codim}_{\infty}(V)=2$. A condição $\overline{M(f) \backslash V} \cap V$ ser limitada segue do fato bem conhecido que qualquer função polinomial complexa satisfaz a condição $a_{f}$ de Thom no infinito ao longo de uma estratificação de Whitney do conjunto dos zeros. Ver, por exemplo, M. Tibăr [Ti].

Além disso, segue do Corolário 3.6 - página 34 que $M(f /\|f\|)$ é limitado, o que completa a prova do Corolário. 


\section{Capítulo 4}

\section{Um Teorema Estrutural para Conjuntos Semi-Algébricos}

\section{Sumário}

4.1 As condições de Milnor $(a)$ e $(b)$ generalizadas . . . . . . . . 41

4.2 Estrutura open book de aplicações polinomiais . . . . . . . . 43

4.3 Uma condição semi-algébrica ... . . . . . . . . . . 44

\subsection{As condições de Milnor $(a)$ e $(b)$ generalizadas}

Neste capítulo apresentaremos um dos principais resultados da Tese. A saber, provaremos uma generalização dos Teoremas de fibrações em esferas: caso global (Teorema 3.12 - página 39) e caso local ([Mil1, Teorema 11.2, pg. 97]). Para tanto, em analogia com as esferas, consideraremos $W^{n-1} \subset \mathbb{R}^{N}$ uma variedade suave compacta sem bordo semi-algébrica de dimensão $n-1$.

Seja $F=\left(f_{1}, \ldots, f_{p}\right): \mathbb{R}^{N} \rightarrow \mathbb{R}^{p}, 2 \leq p \leq n-1<N$ uma aplicação $C^{2}$-semi-algébrica e $\bar{F}:=\frac{F}{\|F\|}: \mathbb{R}^{N} \backslash V(F) \rightarrow S^{p-1}$, em que $V(F):=F^{-1}(0)$, provaremos um critério de existência da estrutura de fibração induzida por $\bar{F}$ (Teorema 4.1 - página 42). Além disso, também mostraremos que a aplicação $G:=\pi \circ F:\left(f_{1}, \ldots, f_{p-1}\right): \mathbb{R}^{N} \rightarrow \mathbb{R}^{p-1}$, em que $\pi$ é a projeção canônica na meta, também define uma fibração suave localmente trivial (Teorema 4.10 - página 47$)$.

Definição 4.1 Sejam $F: \mathbb{R}^{N} \rightarrow \mathbb{R}^{p}$ uma aplicação $C^{2}$-semi-algébrica, $W \subset \mathbb{R}^{N}$ como acima e $V_{W}(F):=V(F) \cap W$.

(1) Se a aplicação $\bar{F}_{\mid W}: W \backslash V_{W}(F) \rightarrow S^{p-1}$ for uma fibração suave localmente trivial, diremos que $\bar{F}_{\mid}$é a fibração estrutural induzida por $F$ em $W$.

(2) Diremos que F satisfaz as condições de Milnor (a) e (b) generalizadas se: 
(a) $\overline{\sum_{F}^{W} \backslash V_{W}(F)} \cap V_{W}(F)=\emptyset$;

(b) $\Sigma_{\bar{F}}^{W}=\emptyset$.

Notações:

- $\Sigma_{F}$ é o conjunto de pontos críticos de $F$;

- $\Sigma_{\bar{F}}$ é o conjunto de pontos críticos de $\bar{F}$;

- $\Sigma_{F}^{W}$ é o conjunto de pontos críticos de $F_{\mid W}$;

- e $\Sigma_{\bar{F}}^{W}$ é o conjunto de pontos críticos de $\bar{F}_{\mid W}$.

Nosso principal resultado desta seção é o seguinte:

Teorema 4.1 (Teorema Estrutural) Se F satisfaz a condição de Milnor (a) generalizada, então são equivalentes:

(i) $\bar{F}_{\mid W}$ é uma fibração estrutural induzida por $F$ em $W$;

(ii) F satisfaz a condição de Milnor (b) generalizada.

DemonstraÇÃo: Sem perda de generalidades, podemos assumir que $W$ é conexo. Além disso, observe inicialmente que a condição de Milnor (b) generalizada vale, se e somente se,

$$
\bar{F}: W \backslash V_{W}(F) \rightarrow S^{p-1}
$$

é uma submersão.

Pelo Lema de seleção da curva local, temos que a condição $(a)$ significa que existe $\delta>0$ suficientemente pequeno e um disco fechado $D_{\delta}$ centrado na origem, tal que

$$
F_{\mid}: F^{-1}\left(D_{\delta} \backslash\{0\}\right) \cap W \rightarrow D_{\delta} \backslash\{0\}
$$

é uma $C^{2}$-submersão sobrejora própria.

$(i) \Rightarrow(i i)$ : Neste caso, temos que $\bar{F}: W \backslash V_{W}(F) \rightarrow S^{p-1}$ é uma fibração suave localmente trivial, em particular, ela é uma submersão. Logo, vale a condição de Milnor $(b)$ generalizada.

$(i i) \Rightarrow(i)$ : Podemos supor que $V_{W}(F)$ é não vazio. De fato, se ele é vazio a condição de Milnor $(a)$ generalizada é sempre satisfeita e pela condição de Milnor $(b)$ generalizada, temos que $\bar{F}: W \rightarrow S^{p-1}$ é uma $C^{2}$-submersão própria e sobrejetora, desde que $S^{p-1}$ é conexo. Portanto, $(i)$ segue do Teorema de Ehresmann.

No caso $V_{W}(F)$ é não vazio, a projeção $\bar{F}: W \backslash V_{W}(F) \rightarrow S^{p-1}$ não é própria e não podemos usar o Teorema de Ehresmann diretamente. Contudo, a aplicação $F: F^{-1}\left(D_{\delta} \backslash\right.$ $\{0\}) \cap W \rightarrow D_{\delta} \backslash\{0\}$ é submersão sobrejetiva e própria. Assim, pelo Teorema de Ehresmann, a aplicação

$$
F_{\mid}: F^{-1}\left(D_{\delta} \backslash\{0\}\right) \cap W \rightarrow D_{\delta} \backslash\{0\}
$$


é uma fibração suave localmente trivial. Agora, podemos compor (4.1) com a projeção radial $\pi_{1}:\left(D_{\delta} \backslash\{0\}\right) \rightarrow S^{p-1}, \pi_{1}(y):=\frac{y}{\|y\|}$ e, por conseguinte, temos que

$$
\bar{F}: F^{-1}\left(D_{\delta} \backslash\{0\}\right) \cap W \rightarrow S^{p-1}
$$

é uma fibração suave localmente trivial.

Da fibração (4.1) temos que a aplicação

$$
\bar{F}: F^{-1}\left(S_{\delta}\right) \cap W \rightarrow S^{p-1}
$$

é também uma fibração suave localmente trivial e portanto sobrejetiva.

Isto implica que a aplicação

$$
\bar{F}: W \backslash F^{-1}\left(\stackrel{\circ}{D}_{\delta}\right) \rightarrow S^{p-1}
$$

é sobrejetiva e própria, em que $\stackrel{\circ}{D}_{\delta}^{p}$ é um disco aberto em $\mathbb{R}^{p}$. Agora, usando a condição de Milnor (b) generalizada, temos que a aplicação (4.4) é uma $C^{2}$-submersão. Portanto, pelo Teorema de Ehresmann temos que esta aplicação é uma fibração suave localmente trivial,

Para completar a prova observe que podemos "colar" as fibrações (4.2) e (4.4) pelo bordo comum $F^{-1}\left(S_{\delta}\right) \cap W$, usando a fibração (4.3), para conseguir a fibração suave localmente trivial desejada.

Observação 4.2 (a) O argumento principal na prova do Teorema anterior é baseado no Teorema de Ehresmann. Portanto, é possivel generalizar as condições (a) e (b) acima e o Teorema 4.1 para $C^{2}$-aplicações e variedades não necessariamente semi-algébricas;

(b) É possivel trocar a subvariedade $W$ por um conjunto compacto semi-algébrico com conjunto singular $\Sigma(W) \neq \emptyset$, se adicionarmos como hipótese a condição $\Sigma(W) \subseteq$ $V_{W}(F)$. Também poderíamos trocar a condição de compacidade de $W$, pela condição de $F$ ser uma aplicação própria em W. Em ambos os casos citados o Teorema $4.1 \mathrm{e}$ sua prova seriam análogas.

\subsection{Estrutura open book de aplicações polinomiais}

A seguir descreveremos como nosso Teorema Estrutural para aplicações semi-algébricas generaliza os análogos do caso local e global.

Considere $F=\left(f_{1}, \ldots, f_{p}\right):\left(\mathbb{R}^{N}, 0\right) \rightarrow\left(\mathbb{R}^{p}, 0\right)$ um germe de aplicação analítica real satisfazendo as hipóteses do [ACT, Teorema 1.3]. Seja $W=\rho^{-1}\left(\epsilon^{2}\right)=S_{\epsilon}^{N-1}$, em que $\rho(x)=\sum_{i=1}^{n} x_{i}^{2}$ e $\epsilon>0$ suficientemente pequeno, $V_{W}(F):=K_{\epsilon}$ o link do germe $V(F)$. O conjunto singular $\Sigma_{F}^{W}$ (e respectivamente, $\Sigma_{\bar{F}}^{W}$ ) é, portanto, o conjunto de Milnor $M(F):=$ $\left\{x \in \mathcal{U}: F \not_{x} \rho\right\}$ (respectivamente, $M(\bar{F}):=\left\{x \in \mathcal{U} \backslash V(F): \bar{F} \not_{x} \rho\right\}$ ). Com estas considerações, temos que as condições de Milnor $(a)$ e $(b)$ generalizadas coincidem com as condições estudadas em [ACT] e, portanto, o Teorema 1.3 de [ACT] é um caso particular do nosso Teorema 4.1 - página 42. 
Para ver o que ocorre no caso global, vamos considerar uma aplicação polinomial real $F=\left(f_{1}, \ldots, f_{p}\right): \mathbb{R}^{N} \rightarrow \mathbb{R}^{p}$, e escolher $W$ como sendo $W:=S_{R}^{N-1}$ com $R \gg 1$. Neste caso, nossas condições $(a)$ e $(b)$ significam que os conjuntos $\overline{M(F) \backslash V} \cap V$ e $M(\bar{F})$ são limitados, assim, o Teorema 3.12 - página 39 é obtido do nosso Teorema 4.1 - página 42.

Exemplo 4.3 Seja $F: \mathbb{R}^{3} \rightarrow \mathbb{R}^{2}$, definida por $F(x, y, z)=\left(x, x^{2}+y\left(x^{2}+y^{2}+z^{2}\right)\right)$. Cálculos diretos mostram que $F$ tem apenas uma singularidade isolada $(0,0,0)$ e, portanto, a condição de Milnor (a) generalizada vale. Considerando $P(x, y, z)=x, Q(x, y, z)=x^{2}+y\left(x^{2}+y^{2}+z^{2}\right)$, pelo Lema 4.5 - página 45 basta analisar o rank da matriz

$$
\left(\begin{array}{c}
\omega(x, y, z) \\
\nabla \rho(x, y, z)
\end{array}\right)_{2 \times 3}
$$

em que $\omega(x, y, z)=P(x, y, z) \nabla Q(x, y, z)-Q(x, y, z) \nabla P(x, y, z)$, para concluir que $M(F /\|F\|)$ é limitado.

Portanto, para $R$ suficientemente grande, $\frac{F}{\|F\|}: S_{R}^{2} \backslash K_{R} \rightarrow S_{1}^{1}$ é uma fibração suave localmente trivial.

\subsection{Uma condição semi-algébrica}

Considerando um germe de aplicação polinomial real $F=\left(f_{1}, \cdots, f_{p}\right):\left(\mathbb{R}^{n}, 0\right) \rightarrow$ $\left(\mathbb{R}^{p}, 0\right)$, tal que $F(0)=0, n \geq p \geq 2$ com singularidade isolada, podemos compor com o germe de projeção $\pi:\left(\mathbb{R}^{p}, 0\right) \rightarrow\left(\mathbb{R}^{p-1}, 0\right)$ para obter um novo germe de aplicação $g:=\pi \circ F:\left(\mathbb{R}^{n}, 0\right) \rightarrow\left(\mathbb{R}^{p-1}, 0\right)$ a qual certamente também tem singularidade isolada na origem.

Em $[\mathrm{AD}]$ os autores provaram um resultado mais geral mostrando que se um germe $F=\left(f_{1}, \cdots, f_{p}\right):\left(\mathbb{R}^{n}, 0\right) \rightarrow\left(\mathbb{R}^{p}, 0\right)$ de aplicação analítica real satisfaz as condições de Milnor $(a)$ e $(b)$ introduzidas por D. Massey em [Ma2], então para todo $I=\left\{i_{1}, \cdots, i_{l}\right\}$, $2 \leq l \leq p$, o germe de aplicação $f_{I}=\left(f_{i_{1}}, \cdots, f_{i_{l}}\right):\left(\mathbb{R}^{n}, 0\right) \rightarrow\left(\mathbb{R}^{l}, 0\right)$ também satisfaz as condições de Milnor $(a)$ e $(b)$.

Nosso próximo objetivo é provar que as condições de Milnor $(a)$ e (b) generalizadas, também são preservadas após considerarmos a composição da aplicação $C^{2}$-semi-algébrica $F=\left(f_{1}, \cdots, f_{p}\right): \mathbb{R}^{N} \rightarrow \mathbb{R}^{p}$ com projeções canônicas na meta. Tal resultado estende de maneira natural todos os resultados obtidos anteriormente nesta direção. Para tanto, considere a seguinte definição motivada no Teorema de Tarski-Seidenberg (Teorema 1.7 página 9):

Definição 4.2 Sejam $F=\left(f_{1}, \ldots, f_{p}\right): X \subset \mathbb{R}^{N} \rightarrow \mathbb{R}^{p}$ uma aplicação $C^{2}$-semi-algébrica, $X \subset \mathbb{R}^{N}$ um conjunto semi-algébrico, $\pi: \mathbb{R}^{p} \rightarrow \mathbb{R}^{p-1}$ a projeção canônica, e suponha que $F$ satisfaz uma certa propriedade $A$. Diremos que $A$ é uma propriedade semi-algébrica, se $\pi \circ F$ também satisfaz a propriedade $A$.

Assim, no que segue, mostraremos que as condições de Milnor $(a)$ e $(b)$ generalizadas são condições semi-algébricas. E, por conseguinte, pelo Teorema Estrutural considerando 
$G:=\pi \circ F=\left(f_{1}, \ldots, f_{p-1}\right): \mathbb{R}^{p} \rightarrow \mathbb{R}^{p-1}, p \geq 3$, teremos que $\bar{G}_{\mid W}$ também define uma fibração suave localmente trivial.

A importante observação abaixo determina os geradores do espaço normal das fibras de $\bar{F}: \mathbb{R}^{N} \backslash V(F) \rightarrow S^{p-1}$.

Observação 4.4 Seja $\pi_{i}: \mathbb{R}^{p} \rightarrow \mathbb{R}^{p-1}$ a projeção $\left(y_{1}, \cdots, y_{p}\right) \mapsto\left(y_{1}, \cdots, \hat{y}_{i}, \ldots, y_{p}\right) . \quad A$ restrição de $\pi \circ \bar{F}$ ao conjunto aberto $\left\{f_{i} \neq 0\right\}$ é dada por:

$$
\begin{aligned}
\pi_{i} \circ \bar{F}: \mathbb{R}^{N} \backslash V(F) & \rightarrow B^{p-1} \subset \mathbb{R}^{p-1} \\
x & \mapsto\left(\bar{f}_{1}(x), \cdots, \widehat{\bar{f}_{i}(x)}, \cdots, \bar{f}_{p}(x)\right)
\end{aligned}
$$

em que $\bar{f}_{i}$ denota a função $\frac{f_{i}}{\|F\|} e \widehat{\bar{f}}_{i}$ significa que omitimos a função $\bar{f}_{i}$, para $i=1, \cdots, p$. Além disso, temos que $\Sigma_{\bar{F}} \cap\left\{f_{i} \neq 0\right\}$ é o conjunto de pontos, em que

$$
\operatorname{rank}\left[\begin{array}{c}
\nabla \bar{f}_{1} \\
\vdots \\
\nabla \bar{f}_{i-1} \\
\nabla \bar{f}_{i+1} \\
\vdots \\
\nabla \bar{f}_{p}
\end{array}\right]<p-1
$$

Veremos abaixo uma forma de obter estes geradores explicitamente. Nesta direção considerando, como em [AT, ACT1], para cada $1 \leq i, j \leq p$ os campos de vetores:

$$
\omega_{i, j}(x)=f_{i}(x) \nabla f_{j}(x)-f_{j}(x) \nabla f_{i}(x)
$$

segue que $\omega_{i, i}(x)=0$ e $\omega_{j, i}(x)=-\omega_{i, j}(x)$. Além disso, temos o seguinte:

Lema 4.5 Se $F=\left(f_{1}, \cdots, f_{p}\right): \mathbb{R}^{n} \rightarrow \mathbb{R}^{p}$ uma aplicação $C^{1}$-semi-algébrica, então para cada $1 \leq j \leq p$, temos que

$$
\nabla\left(\frac{f_{j}}{\|F\|}\right)(x)=\frac{1}{\|F\|^{3}} \sum_{1 \leq k \neq j}^{p} f_{k}(x) \omega_{k, j}(x) .
$$

Observação 4.6 Decorre do Lema anterior que se $x$ não for um ponto singular de $\bar{F}$, então o conjunto de vetores $\left\{\omega_{i, j}(x)\right\}_{1 \leq i<j \leq p}$ gera o espaço normal em $\mathbb{R}^{n} \backslash V(F)$ da $\bar{F}^{-1}(\bar{F}(x))$ em $x$.

Segue da definição a seguinte relação circular.

Lema 4.7 Para todo $1 \leq i<j<k \leq p$ temos:

$$
f_{i} \omega_{j, k}+f_{k} \omega_{i, j}+f_{j} \omega_{k, i}=0,
$$


Como esta relação contém importantes informações sobre o espaço normal da fibra da projeção $\bar{F}: \mathbb{R}^{N} \backslash V(F) \rightarrow S^{p-1}$, vamos denominá-la de equação de Milnor.

Lema 4.8 Dada $F$ como acima, para todo $x$ no conjunto aberto $\left\{f_{1}(x) \neq 0\right\}$, temos a seguinte equação matricial:

$$
\begin{aligned}
& \|F(x)\|^{3 p} \cdot\left(\begin{array}{c}
\nabla\left(\frac{f_{1}}{\|F\|}\right)(x) \\
\nabla\left(\frac{f_{2}}{\|F\|}\right)(x) \\
\vdots \\
\nabla\left(\frac{f_{p}}{\|F\|}\right)(x)
\end{array}\right)_{p \times n}= \\
& \left(\begin{array}{ccccc}
-f_{2} & -f_{3} & \ldots & -f_{p-1} & -f_{p} \\
\frac{f_{1}^{2}+f_{3}^{2}+\cdots+f_{p}^{2}}{f_{1}} & -\frac{f_{2} f_{3}}{f_{1}} & \ldots & -\frac{f_{2} f_{p-1}}{f_{1}} & -\frac{f_{2} f_{p}}{f_{1}} \\
-\frac{f_{2} f_{3}}{f_{1}} & \frac{f_{1}^{2}+f_{2}^{2}+\cdots+f_{p-1}^{2}+f_{p}^{2}}{f_{1}} & \ldots & -\frac{f_{3} f_{p-1}}{f_{1}} & -\frac{f_{3} f_{p}}{f_{1}} \\
\cdots & \cdots & \ldots & \ldots & \cdots \\
-\frac{f_{2} f_{p}}{f_{1}} & -\frac{f_{3} f_{p}}{f_{1}} & \ldots & -\frac{f_{p-1} f_{p}}{f_{1}} & \frac{f_{1}^{2}+f_{2}^{2}+\cdots+f_{p-1}^{2}}{f_{1}}
\end{array}\right)_{p \times(p-1)} \quad\left(\begin{array}{c}
\omega_{1,2}(x) \\
\omega_{1,3}(x) \\
\ldots \\
\omega_{1, p}(x)
\end{array}\right)_{(p-1) \times n}
\end{aligned}
$$

em que a $p \times(p-1)$ matriz tem rank maximal.

DemonstraÇÃo: A igualdade matricial segue diretamente do Lema 4.5 e da igualdade de Milnor.

Agora, para verificarmos que a $p \times(p-1)$ matriz tem rank maximal, considere a $(p-$ $1) \times(p-1)$ submatriz, digamos $A(x)$, obtida da $p \times(p-1)$ matriz acima removendo-se a primeira linha

$$
A(x)=\left(\begin{array}{ccccc}
\frac{f_{1}^{2}+f_{3}^{2}+\cdots+f_{p}^{2}}{f_{1}} & -\frac{f_{2} f_{3}}{f_{1}} & -\frac{f_{2} f_{4}}{f_{1}} & \ldots & -\frac{f_{2} f_{p}}{f_{1}} \\
-\frac{f_{2} f_{3}}{f_{1}} & \frac{f_{1}^{2}+f_{2}^{2}+\cdots+f_{p-1}^{2}+f_{p}^{2}}{f_{1}} & -\frac{f_{3} f_{4}}{f_{1}} & \ldots & -\frac{f_{3} f_{p}}{f_{1}} \\
\cdots & \cdots & \cdots & \ldots & \cdots \\
-\frac{f_{2} f_{p}}{f_{1}} & -\frac{f_{3} f_{p}}{f_{1}} & -\frac{f_{4} f_{p}}{f_{1}} & \ldots & \frac{f_{1}^{2}+f_{2}^{2}+\cdots+f_{p-1}^{2}}{f_{1}}
\end{array}\right)
$$

Como $A(x)$ é uma matriz simétrica, temos que ela é diagonalizável, assim, a multiplicidade algébrica e geométrica coincidem. Agora, usando ferramentas de álgebra linear, podemos provar que $\frac{\|F(x)\|^{2}}{f_{1}(x)}$ é um autovalor com multiplicidade $(p-2)$ e $f_{1}(x)$ é um autovalor com multiplicidade um. Portanto, o determinante de $A(x)$ é igual a $\frac{\|F(x)\|^{2(p-2)}}{f_{1}^{p-3}(x)}$, o qual nunca se anula no conjunto aberto $\left\{f_{1}(x) \neq 0\right\}$. Portanto, o operador linear $A(x)$ é injetivo e os ranks da $p \times n$ matriz do lado esquerdo e da $(p-1) \times n$ matriz do lado direito são iguais, o que completa a prova.

Observação 4.9 O Lema acima ainda é válido com a mesma demonstração, se trocarmos o aberto $\left\{f_{1}(x) \neq 0\right\}$ pelo aberto $\left\{f_{i}(x) \neq 0\right\}$, para cada $i=2, \cdots, p$. Neste caso, a $i-$ ésima 
linha da matriz $p \times(p-1)$ é dada por

$$
\left\{\begin{array}{c}
a_{i k}=-f_{k}, \text { se } k<i \\
a_{i k}=-f_{k+1}, \text { se } k \geq i
\end{array}\right.
$$

Agora, removendo esta linha da matriz, obtemos uma $(p-1) \times(p-1)$ matriz simétrica tal que os elementos na diagonal são dados por

$$
\left\{\begin{array}{c}
a_{j j}=\sum_{1 \leq k \neq j}^{p} \frac{f_{k}^{2}}{f_{i}}, \text { se } j<i \\
a_{j j}=\sum_{1 \leq k \neq j+1}^{p} \frac{f_{k}^{2}}{f_{i}}, \text { se } j \geq i
\end{array}\right.
$$

e os elementos acima da diagonal são dados por

$$
\left\{\begin{array}{c}
a_{l k}=-\frac{f_{l} f_{k}}{f_{i}}, \text { se } k<i \\
a_{l k}=-\frac{f_{l} f_{k+1}}{f_{i}}, \text { se } k \geq i
\end{array}\right.
$$

$A(p-1) \times n$ matriz neste caso toma a seguinte forma

$$
\left(\begin{array}{c}
\omega_{i, 1} \\
\omega_{i, 2} \\
\vdots \\
\omega_{i, i-1} \\
\omega_{i, i+1} \\
\vdots \\
\omega_{i, p}
\end{array}\right)_{(p-1) \times n}
$$

Agora podemos provar que as condições de Milnor $(a)$ e $(b)$ generalizadas são semialgébricas.

Teorema 4.10 Sejam $F=\left(f_{1}, \ldots, f_{p}\right): \mathbb{R}^{N} \rightarrow \mathbb{R}^{p}$ uma aplicação $C^{2}$-semi-algébrica, $p \geq 3$, e $\pi: \mathbb{R}^{p} \rightarrow \mathbb{R}^{p-1},\left(y_{1}, \cdots, y_{p}\right) \mapsto\left(y_{1}, \cdots, \hat{y}_{i}, \cdots, y_{p}\right)$ a projeção canônica. Se F satisfaz as condições de Milnor $(a)$ e (b) generalizadas, então $\pi \circ F$ também satisfaz.

Demonstração: Como antes denotemos a aplicação composição $\pi \circ F$ por $G=\left(f_{1}, \ldots, f_{p-1}\right)$ e por $\bar{G}=\frac{G}{\|G\|}$. 
Inicialmente, mostraremos que a condição de Milnor $(b)$ generalizada vale para $G$. Para tanto, vamos assumir que existe $x \in \Sigma_{\bar{G}}^{W}$. Então, existe $i \in\{1, \cdots, p-1\}$, tal que $f_{i}(x) \neq 0$. Como, por hipótese, $W \subset \mathbb{R}^{N}$ é uma variedade suave semi-algébrica de dimensão $n-1$, temos que existe uma vizinhança de $x$ tal que $W:=\left\{h_{1}(x)=0, \cdots, h_{N-n+1}(x)=0\right\}$, em que $\left\{h_{1}, \cdots, h_{N-n+1}\right\}$ são funções suaves semi-algébricas e 0 é valor regular da aplicação $\left(h_{1}, \cdots, h_{N-n+1}\right): \mathbb{R}^{N} \rightarrow \mathbb{R}^{N-n+1}$. Pela observação 4.4 , no ponto $x$, temos que

$$
\operatorname{rank}\left[\begin{array}{c}
\nabla h_{1}(x) \\
\vdots \\
\nabla h_{N-n+1}(x) \\
\nabla \bar{f}_{1}(x) \\
\vdots \\
\nabla \bar{f}_{i-1}(x) \\
\nabla \bar{f}_{i+1}(x) \\
\vdots \\
\nabla \bar{f}_{p-1}(x)
\end{array}\right]<N-n+1+p-2,
$$

Assim, adicionando-se na última linha da matriz anterior o vetor $\nabla \bar{f}_{p}(x)$, segue que:

$$
\operatorname{rank}\left[\begin{array}{c}
\nabla h_{1}(x) \\
\vdots \\
\nabla h_{N-n+1}(x) \\
\nabla \bar{f}_{1}(x) \\
\vdots \\
\nabla \bar{f}_{i-1}(x) \\
\nabla \bar{f}_{i+1}(x) \\
\vdots \\
\nabla \bar{f}_{p}(x)
\end{array}\right]<N-n+1+p-1
$$

E pela observação 4.4, temos que $x \in \Sigma_{\bar{F}}^{W}$, o que é uma contradição com a hipótese $\Sigma_{\bar{F}}^{W}=\emptyset$. Logo $G$ satisfaz a condição $(b)$.

Agora, provaremos que $G$ também satisfaz a condição de Milnor (a) generalizada, isto é, temos que mostrar que

$$
\overline{\Sigma_{G}^{W} \backslash V_{W}(G)} \cap V_{W}(G)=\emptyset .
$$

Para tanto, observe inicialmente, que $\Sigma_{G}^{W} \subset \Sigma_{F}^{W}$ e $V_{W}(F) \subset V_{W}(G)$. Assim,

$$
\Sigma_{G}^{W} \backslash V_{W}(G) \subset \Sigma_{F}^{W} \backslash V_{W}(F) \Rightarrow \overline{\Sigma_{G}^{W} \backslash V_{W}(G)} \subset \overline{\Sigma_{F}^{W} \backslash V_{W}(F)} .
$$

Logo, vale a seguinte inclusão:

$$
\overline{\Sigma_{G}^{W} \backslash V_{W}(G)} \cap V_{W}(F) \subset \overline{\Sigma_{F}^{W} \backslash V_{W}(F)} \cap V_{W}(F) .
$$

Como $\overline{\Sigma_{F}^{W} \backslash V_{W}(F)} \cap V_{W}(F)=\emptyset$, temos que $\overline{\Sigma_{G}^{W} \backslash V_{W}(G)} \cap V_{W}(F)=\emptyset$. Assim, para completarmos a prova da condição $(b)$ para $G$, resta verificarmos o que ocorre quando consideramos o conjunto $V_{W}(G) \backslash V_{W}\left(f_{p}\right)$, isto é, se $f_{p} \neq 0$. Mas, pela observação 4.4 , temos que 
$x \in \Sigma_{\bar{F}}^{W} \backslash V_{W}\left(f_{p}\right)$ se, e somente se,

$$
\operatorname{rank}\left[\begin{array}{c}
\nabla h_{1}(x) \\
\vdots \\
\nabla h_{N-n+1}(x) \\
\nabla \bar{f}_{1}(x) \\
\vdots \\
\nabla \bar{f}_{p-1}(x)
\end{array}\right]<N-n+1+p-1
$$

Por outro lado, em $V_{W}(G)$, para $i=1, \ldots, p-1$, temos que

$$
\nabla \bar{f}_{i}=\frac{1}{\|F\|^{2}}\left(\|F\| \nabla f_{i}-f_{i} \nabla\|F\|\right)=\frac{1}{\|F\|} \nabla f_{i}
$$

Consequentemente,

$$
x \in \Sigma_{\bar{F}}^{W} \cap\left\{f_{p} \neq 0\right\} \Leftrightarrow x \in \Sigma_{G}^{W} \cap\left\{f_{p} \neq 0\right\} .
$$

Portanto,

$$
\Sigma_{\bar{F}}^{W} \cap\left[V_{W}(G) \backslash V_{W}\left(f_{p}\right)\right]=\Sigma_{G}^{W} \cap\left[V_{W}(G) \backslash V_{W}\left(f_{p}\right)\right]
$$

Agora, note que podemos escrever

$$
\begin{aligned}
\overline{\Sigma_{G}^{W} \backslash V_{W}(G)} \cap V_{W}(G) & =\overline{\sum_{G}^{W} \backslash V_{W}(G)} \cap\left[V_{W}(G) \backslash V_{W}\left(f_{p}\right) \cup V_{W}(F)\right] \\
& =\overline{\Sigma_{G}^{W} \backslash V_{W}(G)} \cap V_{W}(F) \bigcup \overline{\Sigma_{G}^{W} \backslash V_{W}(G)} \cap\left[V_{W}(G) \backslash V_{W}\left(f_{p}\right)\right] .
\end{aligned}
$$

Como $\Sigma_{G}^{W}$ é fechado e $\overline{\Sigma_{G}^{W} \backslash V_{W}(G)} \cap V_{W}(F)=\emptyset$, concluímos que

$$
\overline{\Sigma_{G}^{W} \backslash V_{W}(G)} \cap V_{W}(G) \subset \Sigma_{G}^{W} \cap\left[V_{W}(G) \backslash V_{W}\left(f_{p}\right)\right] .
$$

Assim, por (4.8), temos que

$$
\overline{\Sigma_{G}^{W} \backslash V_{W}(G)} \cap V_{W}(G) \subset \Sigma_{\bar{F}}^{W} \cap\left[V_{W}(G) \backslash V_{W}\left(f_{p}\right)\right] .
$$

Mas, pela condição de Milnor $(b)$ generalizada para $F$, o lado direito desta inclusão é vazio, logo

$$
\overline{\Sigma_{G}^{W} \backslash V_{W}(G)} \cap V_{W}(G)=\emptyset .
$$

E, portanto, vale a condição de Milnor (b) generalizada para $G$, como queríamos mostrar.

Observação 4.11 Segue diretamente como uma aplicação do Teorema Estrutural que a aplicação $\bar{G}: W \backslash V_{W}(G) \rightarrow S^{p-2}$ é também uma fibração suave localmente trivial para todo $p \geq 3$. 


\section{Capítulo 5}

\section{Topologia da Fibra de Milnor no Infinito}

\section{Sumário}

5.1 Relação entre as fibras $M_{F}$ e $M_{G} \ldots \ldots \ldots \ldots$

5.2 A característica de Euler das fibras e dos links relativos . . . . 57

Considerando um germe de aplicação analítica $F:\left(f_{1}, \cdots, f_{p}\right):\left(\mathbb{R}^{n}, 0\right) \rightarrow\left(\mathbb{R}^{p}, 0\right)$, $n>p \geq 2 \operatorname{com} \Sigma_{F}=\{0\}$, Milnor mostrou que para cada $\epsilon>0$ suficientemente pequeno, existe um $\delta>0,0<\delta \ll \epsilon$, tal que, a aplicação

$$
F_{\mid}: B_{\epsilon}^{n} \cap F^{-1}\left(S_{\eta}^{p-1}\right) \rightarrow S_{\eta}^{p-1},
$$

é uma fibração suave localmente trivial. Além disso, foi usado um campo de vetores radial conveniente em $B_{\epsilon}^{n} \backslash V$ para "inflar" o tubo $B_{\epsilon}^{n} \cap F^{-1}\left(S_{\eta}^{p-1}\right)$ para a esfera $S_{\epsilon}^{n-1}$ mantendo o bordo $S_{\epsilon}^{n-1} \cap F^{-1}\left(S_{\eta}^{p-1}\right)$ fixo, obtendo uma fibração localmente trivial

$$
S_{\epsilon}^{n-1} \backslash N\left(K_{\epsilon}\right) \rightarrow S_{\eta}^{p-1}
$$

em que $N\left(K_{\epsilon}\right)$ é uma vizinhança tubular fechada de $K_{\epsilon}$ em $S_{\epsilon}^{n-1}$

Considerando $p \geq 3$ e o germe de aplicação $G:=\left(f_{1}, \cdots, f_{p-1}\right):\left(\mathbb{R}^{n}, 0\right) \rightarrow\left(\mathbb{R}^{p-1}, 0\right)$, obtida de $F$ omitindo a última função coordenada, em [Mil1, pg. 100] Milnor conjecturou:

Conjetura 5.1 Sejam $\widetilde{M}_{F}$ e $\widetilde{M}_{G}$ as fibras da fibração 5.2 associadas a $F$ e $G$, respectivamente. A fibra $\widetilde{M}_{G}$ é homeomorfa ao produto da fibra $\widetilde{M}_{F}$ com o intervalo unitário.

Assumindo as condições $(a)$ e $(b)$ de Massey para $p \geq 2$ em [AD], os autores, usando ferramentas da Teoria de Morse e da Teoria de Singularidades, responderam positivamente esta conjectura para a fibra da fibração no tubo de Milnor (5.1). O resultado obtido por eles foi o seguinte:

Teorema 5.2 [AD, Teorema 6.2, pg. 11] Sejam $\mathcal{M}_{F}$ e $\mathcal{M}_{G}$ as fibras da fibração no tubo de Milnor (5.1) associadas a $F$ e $G$, respectivamente. Então $\mathcal{M}_{G}$ é homeomorfa a $\mathcal{M}_{F} \times[-1,1]$. 
Na referência [DAYA, seção 5 , pg. 16] os autores provaram que se $F$ satisfaz as condições de Milnor $(a)$ e $(b)$ definidas por D. Massey e $M(F /\|F\|)$ for vazio, então as fibras da fibração 5.1 e da fibração $\frac{F}{\|F\|}: S_{\epsilon}^{n-1} \backslash K_{\epsilon} \rightarrow S^{p-1}$ são homotopicamente equivalentes.

De forma geral, podemos considerar este problema para $W^{n-1} \subset \mathbb{R}^{N}$ e $F=\left(f_{1}, \cdots, f_{p}\right)$ : $\mathbb{R}^{N} \rightarrow \mathbb{R}^{p}, 2 \leq p \leq n-1<N$ uma aplicação $C^{2}$-semi-algébrica, como no capítulo anterior. Ou seja, já vimos que as condições de Milnor $(a)$ e $(b)$ generalizadas são semi-algébricas (Lema 4.10 - página 47), logo, denotando por $M_{F}$ a fibra de $\bar{F}: W \backslash V_{W}(F) \rightarrow S^{p-1}$ e por $M_{G}$ a fibra de $\bar{G}: W \backslash V_{W}(G) \rightarrow S^{p-2}$ é também natural questionar como elas estão relacionadas.

\subsection{Relação entre as fibras $M_{F}$ e $M_{G}$}

Nesta seção nosso principal resultado é:

Teorema 5.3 A fibra $M_{G}$ é homotopicamente equivalente a fibra $M_{F}$.

Para provarmos o Teorema acima precisaremos de algumas definições e resultados preliminares.

Observação 5.4 Sem perda de generalidades, podemos considerar

$$
M_{F}=\bar{F}^{-1}(1,0, \cdots, 0) \cap\left(W \backslash V_{W}(F)\right) .
$$

Assim, podemos escrever

$$
M_{F}=\left\{f_{1}>0, f_{2}=\cdots=f_{p}=0\right\} \cap W .
$$

Analogamente,

$$
M_{G}=\left\{f_{1}>0, f_{2}=\cdots=f_{p-1}=0\right\} \cap W .
$$

Com isto, temos que $\overline{M_{F}}=M_{F} \cup V_{W}(F)$ e que $V_{W}(F)=\overline{M_{F}} \cap\left\{f_{1}=0\right\}$.

A definição a seguir é motivada pela referência [Th].

Definição 5.1 Sejam $M$ uma variedade suave e $f: M \rightarrow \mathbb{R}$ uma função suave e positiva. Diremos que $f$ é uma "carpeting function" para $M$, se existe um $\delta>0$ tal que em $(0, \delta]$ não existem valores críticos de $f$.

Lema 5.5 As funções $f_{1 \mid M_{F}}$ e $f_{1 \mid M_{G}}$ são carpeting functions para $M_{F}$ e $M_{G}$, respectivamente. 
DemonstraçÃo: Segue da observação 5.4 acima que a função $f_{1}$ é estritamente positiva em $M_{F}$. Vamos provar que existe $\delta>0$ tal que $f_{1}$ é uma submersão em $M_{F} \cap\left(f_{1}\right)^{-1}((0, \delta])$. Assumiremos que isto não ocorre, e, neste caso, podemos encontrar uma sequência $\left\{\delta_{n}\right\}_{n \in \mathbb{N}}$ tal que $\delta_{n}>0, \delta_{n} \rightarrow 0$ e $\left\{f_{1}=\delta_{n}\right\}$ não intersecta $V_{W}\left(f_{2}, \cdots, f_{p}\right)$ transversalmente. Isto implica que existe $y_{n}$ em $\left\{f_{1}=\delta_{n}\right\}$ tal que $y_{n} \in \Sigma_{F}^{W} \backslash V_{W}(F)$. Mas, como $W$ é compacto, temos que $\overline{\sum_{F}^{W} \backslash V_{W}(F)} \cap V_{W}(F) \neq \emptyset$ o que contradiz a condição de Milnor $(a)$ generalizada para $F$. Portanto, $f_{1}$ é carpeting function para $M_{F}$. A mesma prova vale para $M_{G}$, e, consequentemente, o Lema está provado.

Corolário 5.6 A fibra $M_{F}$ (resp. $M_{G}$ ) é homotopicamente equivalente a $\left\{f_{1} \geq \delta, f_{2}=\cdots=\right.$ $\left.f_{p}=0\right\} \cap W$ (resp. $\left.\left\{f_{1} \geq \delta, f_{2}=\cdots=f_{p-1}=0\right\} \cap W\right)$.

Demonstração: Vamos considerar a fibra $M_{F}$. A prova para $M_{G}$ é análoga.

Como $f_{1}$ é uma carpeting function para $M_{F}$, segue do Lema 1.19 - página 13 que a fibra $M_{F}$ é difeomorfa a $\left\{f_{1}>\delta, f_{2}=\cdots=f_{p}=0\right\} \cap W$. O resultado segue do fato bem conhecido que toda variedade com bordo é homotopicamente equivalente a sua parte aberta.

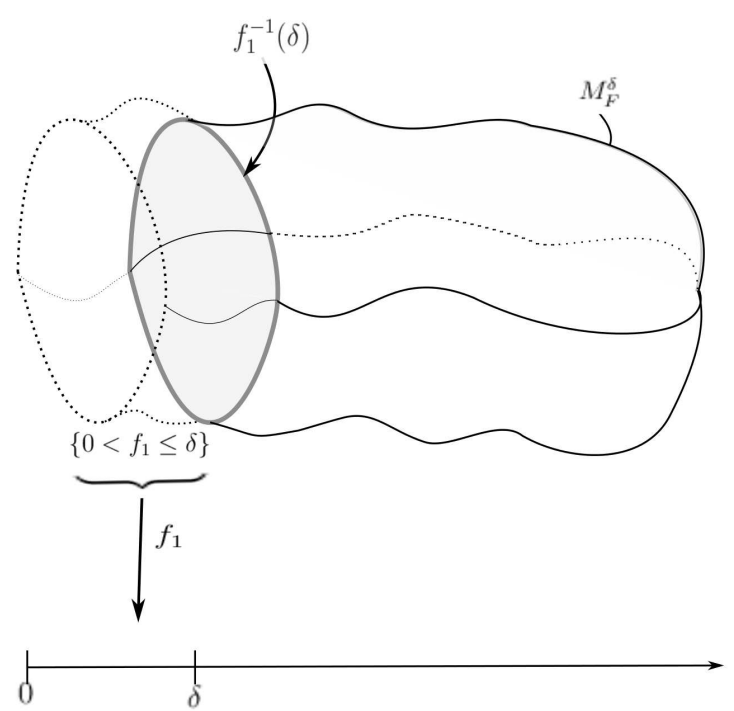

Vamos denotar por $M_{F}^{\delta}$ e $M_{G}^{\delta}$ as variedades com bordo $\left\{f_{1} \geq \delta, f_{2}=\cdots=f_{p}=0\right\} \cap$ $W$ e $\left\{f_{1} \geq \delta, f_{2}=\cdots=f_{p-1}=0\right\} \cap W$, respectivamente. Desta forma, observe que $M_{F}^{\delta}=M_{G}^{\delta} \cap\left\{\bar{f}_{p}=0\right\}$, em que $\bar{f}_{p}$ denota a função $\frac{f_{p}}{\|F\|}$.

No que segue vamos estudar os pontos críticos de $\bar{f}_{p_{\mid M_{G}^{\delta}}}$ usando as ferramentas da Teoria de Morse para variedades com bordo.

Nestas condições, temos a seguinte: 
Observação 5.7 A função $\bar{f}_{p \mid W \cap V\left(f_{2}, \cdots, f_{p-1}\right)}$ não tem pontos críticos no aberto $\left\{f_{1}(x) \neq 0\right\}$.

Demonstração: De fato, suponhamos que $x$ seja um ponto crítico de $\bar{f}_{p \mid W \cap V\left(f_{2}, \cdots, f_{p-1}\right)}$ no conjunto aberto $\left\{f_{1}(x) \neq 0\right\}$. Então, pela observação 4.4 - página 45 , temos que

$$
\operatorname{rank}\left[\begin{array}{c}
\nabla h_{1}(x) \\
\vdots \\
\nabla h_{N-n+1}(x) \\
\nabla f_{2}(x) \\
\vdots \\
\nabla f_{p-1}(x) \\
\nabla \bar{f}_{p}(x)
\end{array}\right]<N-n+1+p-1 .
$$

Mas, em $V\left(f_{2}, \cdots, f_{p-1}\right)$, pelo Lema 4.5 - página 45 , temos que $\nabla \bar{f}_{i}(x)=\frac{1}{\|F(x)\|} \nabla f_{i}(x)$, pois $f_{i}(x)=0$, para $i \in\{2, \cdots, p-1\}$. Assim, podemos reescrever a matriz anterior, da seguinte forma:

$$
\operatorname{rank}\left[\begin{array}{c}
\nabla h_{1}(x) \\
\vdots \\
\nabla h_{N-n+1}(x) \\
\nabla \bar{f}_{2}(x) \\
\vdots \\
\nabla \bar{f}_{p-1}(x) \\
\nabla \bar{f}_{p}(x)
\end{array}\right]<N-n+1+p-1
$$

Como $f_{1}(x) \neq 0$, pela observação 4.4 , temos que a desigualdade acima mostra que $x$ pertence a $\Sigma_{\bar{F}}^{W}$, o qual é vazio, pela condição de Milnor (b) generalizada para $F$. Assim, $\bar{f}_{p \mid W \cap V\left(f_{2}, \cdots, f_{p-1}\right)}$ não tem pontos críticos em $\left\{f_{1}(x) \neq 0\right\}$, como queríamos mostrar.

Nosso próximo passo é investigar os pontos críticos de $\bar{f}_{p}$ restritos a

$$
\partial M_{G}^{\delta}=\left\{f_{1}=\delta, f_{2}=\cdots=f_{p-1}=0\right\} \cap W
$$

Lema 5.8 Para $\delta$ suficientemente pequeno, os pontos críticos de $\bar{f}_{p \mid \partial M_{G}^{\delta}}$ são corretos e pertencem $a\left\{f_{p} \neq 0\right\}$.

DemonstraÇÃo: Denotemos por $\psi=\left(f_{2}, \cdots, f_{p-1}\right)$ e observe que os pontos críticos de $\bar{f}_{p \mid \partial M_{G}^{\delta}}$ são corretos, pois $\bar{f}_{p \mid W \cap V(\psi)}$ não tem pontos críticos em $\left\{f_{1} \neq 0\right\}$. Além disso, como $f_{1}$ é uma carpeting function para $M_{F}$, temos que existe $\delta$ suficientemente pequeno, tal que 
se $x \in M_{F} \cap\left(f_{1}\right)^{-1}((0, \delta])$, vale que,

$$
\operatorname{rank}\left[\begin{array}{c}
\nabla h_{1}(x) \\
\vdots \\
\nabla h_{N-n+1}(x) \\
\nabla f_{1}(x) \\
\vdots \\
\nabla f_{p-1}(x) \\
\nabla f_{p}(x)
\end{array}\right]=N-n+1+p .
$$

Em particular, em $M_{F} \cap\left\{f_{1}=\delta\right\}$, temos que a seguinte igualdade é satisfeita:

$$
\nabla \bar{f}_{p}=\delta^{-1} \nabla f_{p}
$$

pois $\nabla \bar{f}_{p}=\frac{1}{\|F\|} \nabla f_{p}$ em $M_{F} \cap\left\{f_{1}=\delta\right\}$. Trocando $\nabla f_{p}(x)$ por $\nabla \bar{f}_{p}(x)$ na matriz acima, concluímos que os pontos críticos de $\bar{f}_{p \mid \partial M_{G}^{\delta}}$ estão em $\left\{f_{p} \neq 0\right\}$.

Lema 5.9 Se $\delta$ é suficientemente pequeno, então os pontos críticos de $\bar{f}_{p \mid \partial M_{G}^{\delta}}$ em $\left\{f_{p} \neq 0\right\}$ apontam para o exterior (resp. apontam para o interior) de $M_{G}^{\delta}$, se e somente se, eles estão em $\left\{f_{p}>0\right\}$ (resp. $\left.\left\{f_{p}<0\right\}\right)$.

DemonstraÇÃo: Vamos supor que o resultado é falso. Então, para cada $\delta>0$ suficientemente pequeno, podemos encontrar um ponto crítico $x_{\delta}$ de $\bar{f}_{p \mid \partial M_{G}^{\delta}}$ em $\left\{f_{p}>0\right\}$ que aponta para o interior. Portanto, temos que existe $\lambda\left(x_{\delta}\right)>0$ tal que

$$
\nabla \bar{f}_{p \mid V(\psi) \cap W}\left(x_{\delta}\right)=\lambda\left(x_{\delta}\right) \nabla f_{1 \mid V(\psi) \cap W}\left(x_{\delta}\right) .
$$

Pelo Lema de seleção da curva local, temos que existe uma curva analítica

$$
\gamma:[0, \nu[\rightarrow V(\psi) \cap W
$$

tal que $f_{1}(\gamma(0))=0$, e para $\left.t \in\right] 0, \nu[$ :

- $f_{p}(\gamma(t))>0$,

- $f_{1}(\gamma(t))>0$,

- $\nabla \bar{f}_{p \mid V(\psi) \cap W}(\gamma(t))=\lambda(\gamma(t)) \nabla f_{1 \mid V(\psi) \cap W}(\gamma(t)) \operatorname{com} \lambda(\gamma(t))>0$.

Portanto, temos que

$$
\begin{aligned}
\left(\bar{f}_{p} \circ \gamma\right)^{\prime}(t) & =\left\langle\nabla \bar{f}_{p \mid V(\psi) \cap W}(\gamma(t)), \gamma^{\prime}(t)\right\rangle \\
& =\lambda(\gamma(t))\left\langle\nabla f_{1 \mid V(\psi) \cap W}(\gamma(t)), \gamma^{\prime}(t)\right\rangle \\
& =\lambda(\gamma(t))\left(f_{1} \circ \gamma\right)^{\prime}(t) .
\end{aligned}
$$


Afirmamos que $\left(f_{1} \circ \gamma\right)^{\prime}>0$. Pois caso contrário, ou seja, se $\left(f_{1} \circ \gamma\right)^{\prime} \leq 0$, teríamos que $f_{1} \circ \gamma$ seria não crescente. Mas, como $f_{1}(\gamma(t))$ tende a 0 quando $t$ tende a 0 , isto implicaria que $f_{1}(\gamma(t)) \leq 0$, o que é impossível.

Logo, $\left(\bar{f}_{p} \circ \gamma\right)^{\prime}>0$ o que implica que $\bar{f}_{p} \circ \gamma$ é estritamente crescente.

Vamos avaliar $f_{p}(\gamma(0))$. Se $f_{p}(\gamma(0))=0$, então $\gamma(0) \in V_{W}(F)$. Além disso, sabemos que

$$
\nabla \bar{f}_{p}(\gamma(t))=\frac{1}{\|F(\gamma(t))\|^{3}} f_{1}^{2}(\gamma(t)) \nabla f_{p}(\gamma(t))-f_{p}(\gamma(t)) f_{1}(\gamma(t)) \nabla f_{1}(\gamma(t))
$$

isto implica que $\gamma(t)$ também pertence a $\Sigma_{F}^{W} \backslash V_{W}(F)$ para $t>0$, desde que $\gamma(t)$ é um

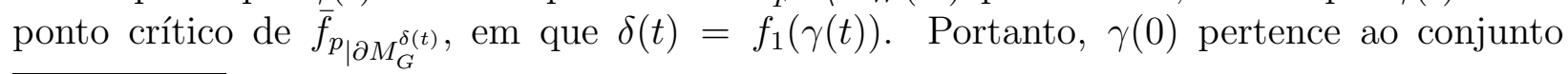
$\overline{\Sigma_{F}^{W} \backslash V_{W}(F)} \cap V_{W}(F)$ o qual é vazio. Consequentemente, $f_{p}(\gamma(0))>0$ e $\bar{f}_{p}(\gamma(0))=1$, pois $f_{1}(\gamma(0))=\ldots=f_{p-1}(\gamma(0))=0$.

Como $\bar{f}_{p} \circ \gamma(t)$ tende para 1 quando $t$ tende para 0 , então a monotonicidade de $\bar{f}_{p} \circ \gamma$ nos dá que $\bar{f}_{p}(\gamma(t))>1$, o que contradiz o fato que $\left|\bar{f}_{p}\right| \leq 1$. 52 .

Com o exposto acima, temos todos os ingredientes para a prova do Teorema 5.3 - página

DemonstraÇÃo: (Teorema 5.3 - página 52). Pelo exposto acima, temos que 0 é um valor regular da função $f_{p}: M_{G}^{\delta} \rightarrow \mathbb{R}$. Como $M_{G}^{\delta}$ é uma variedade com bordo, temos que $M_{G}^{\delta} \cap$ $\left\{f_{p} \geq 0\right\}$ (resp. $M_{G}^{\delta} \cap\left\{f_{p} \leq 0\right\}$ ) é uma variedade com corner, cujo corner é dado por $\partial M_{G}^{\delta} \cap\left\{f_{p}=0\right\}$ (conforme página 15). Além disso, considerando a função $f_{p}: M_{G}^{\delta} \cap\left\{f_{p} \geq\right.$ $0\} \rightarrow \mathbb{R}$ (resp. $f_{p}: M_{G}^{\delta} \cap\left\{f_{p} \leq 0\right\} \rightarrow \mathbb{R}$ ) podemos aplicar a Teoria de Morse para variedades com bordo, visto que os pontos críticos de $f_{p \mid M_{G}^{\delta} \cap\left\{f_{p} \geq 0\right\}}$ (resp. $f_{p \mid M_{G}^{\delta} \cap\left\{f_{p} \leq 0\right\}}$ ) não são pontos de corner. Portanto, segue do Lema 1.21 - página 14 que $M_{G}^{\delta} \cap\left\{f_{p} \geq 0\right\} \sim M_{F}^{\delta} \times[0,1]$ e que $M_{G}^{\delta} \cap\left\{f_{p} \leq 0\right\} \sim M_{F}^{\delta} \times[-1,0]$. Logo, $M_{G}^{\delta} \sim M_{F}^{\delta} \times[-1,1]$, pois ela é homeomorfa a colagem de $M_{G}^{\delta} \cap\left\{f_{p} \geq 0\right\}$ e $M_{G}^{\delta} \cap\left\{f_{p} \leq 0\right\}$ ao longo de $M_{G}^{\delta} \cap\left\{f_{p}=0\right\}=M_{F}^{\delta}$. Usando o Corolário 5.6 - página 53, finalizamos a prova do Teorema.

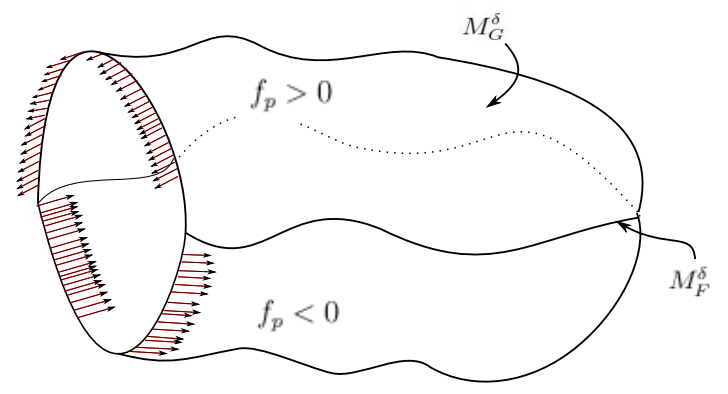

Vamos considerar o caso particular em que $p=2$, isto é, $F=\left(f_{1}, f_{2}\right): \mathbb{R}^{N} \rightarrow \mathbb{R}^{2}$ uma aplicação $C^{2}$ semi-algébrica satisfazendo as condições de Milnor $(a)$ e $(b)$ generalizadas. Denote por $M_{f_{1}}^{+}=\left\{x \in W: f_{1}>0\right\}$ e por $M_{f_{1}}^{-}=\left\{x \in W: f_{1}<0\right\}$. 
Teorema 5.10 A fibra $M_{F}$ é homotopicamente equivalente a $M_{f_{1}}^{+}$e $M_{f_{1}}^{-}$.

Demonstração: A prova é a mesma do Teorema 5.3. Precisamos checar que $f_{1}$ é uma carpeting function para $M_{f_{1}}^{+}$e $-f_{1}$ é uma carpeting function para $M_{f_{1}}^{-}$. Mas $f_{1 \mid W}: W \rightarrow \mathbb{R}$ tem um número finito de valores críticos, assim existe um $\delta>0$, tal que $] 0, \delta]$ e $[-\delta, 0[$, não contém qualquer valor crítico de $f_{1 \mid W}$.

Seja $F=\left(f_{1}, \cdots, f_{p}\right): \mathbb{R}^{N} \rightarrow \mathbb{R}^{p}, 2 \leq p \leq n-1$, uma aplicação $C^{2}$ semi-algébrica satisfazendo as condições de Milnor $(a)$ e $(b)$ generalizadas. Vamos considerar $l \in\{1, \cdots, p\}$ e $I=\left\{i_{1}, \cdots, i_{l}\right\}$ uma lista formada por $l$ inteiros distintos de $\{1, \cdots, p\}$ e seja $f_{I}$ a aplicação semi-algébrica $\left(f_{i_{1}}, \cdots, f_{i_{l}}\right): \mathbb{R}^{N} \rightarrow \mathbb{R}^{l}$.

Sabemos que se $l \geq 2$, então a aplicação $f_{I}$ satisfaz as condições de Milnor $(a)$ e $(b)$ generalizadas. Vamos denotar também por $M_{f_{I}}$ a fibra de $\bar{f}_{I \mid W \backslash V_{W}\left(f_{I}\right)}$.

Corolário 5.11 Sejam $l \in\{2, \cdots, p\}$ e $I=\left\{i_{1}, \cdots, i_{l}\right\}$ como acima. Então, as fibras $M_{f_{I}}$ e $M_{F}$ são homotopicamente equivalentes.

DemonstraÇão: É suficiente aplicar o Teorema 5.3.

Vamos examinar o que acontece quando $l=1$. Pelo exposto anteriormente, temos o seguinte.

Corolário 5.12 Para todo $j \in\{1, \cdots, p\}$, as fibras $M_{f_{j}}^{+}$e $M_{f_{j}}^{-}$são homotopicamente equivalentes a fibra $M_{F}$.

DEmonstraÇÃo: É suficiente aplicar o Teorema 5.10.

\subsection{A característica de Euler das fibras e dos links re- lativos}

Nesta seção, provaremos algumas fórmulas relacionando a característica de Euler dos conjuntos $V_{W}\left(f_{I}\right)$, os quais chamaremos de links relativos (ou simplesmente, links) com a característica de Euler da fibra $M_{F}$. Como uma consequência obteremos uma obstrução para estes links sejam homotopicamente equivalentes após as projeções.

Vamos manter as notações e hipóteses para $F$ como na seção anterior.

Proposição 5.13 Temos que $\chi\left(V_{W}(G)\right)-\chi\left(V_{W}(F)\right)=(-1)^{n-p} 2 \chi\left(M_{F}\right)$. 
DemonstraÇÃo: Vamos escrever

$$
M_{F}^{+}=\left\{f_{1}=f_{2}=\cdots=f_{p-1}=0, f_{p}>0\right\} \cap W=V_{W}(G) \cap\left\{f_{p}>0\right\},
$$

e

$$
M_{F}^{-}=\left\{f_{1}=f_{2}=\cdots=f_{p-1}=0, f_{p}<0\right\} \cap W=V_{W}(G) \cap\left\{f_{p}<0\right\} .
$$

Observe que estes dois conjuntos são difeomorfos a fibra $M_{F}$. Além disso, sabemos, pelo Lema 5.5 - página 52 , que $f_{p}$ é uma carpeting function para $M_{F}^{+}$e $-f_{p}$ é uma carpeting function para $M_{F}^{-}$. Portanto, pelo Corolário 5.6 - página 53 , temos que existe $0<\delta \ll 1$ tal que:

(a) $M_{F}^{+}$é homotopicamente equivalente a $V_{W}(G) \cap\left\{f_{p} \geq \delta\right\}$;

(b) e $M_{F}^{-}$é homotopicamente equivalente a $V_{W}(G) \cap\left\{f_{p} \leq-\delta\right\}$.

Por outro lado, pela propriedade aditiva da característica de Euler, temos que:

$$
\begin{gathered}
\chi\left(V_{W}(G)\right)=\chi\left(V_{W}(G) \cap\left\{f_{p} \geq \delta\right\}\right)+\chi\left(V_{W}(G) \cap\left\{f_{p} \leq-\delta\right\}\right)+ \\
\chi\left(V_{W}(G) \cap\left\{-\delta \leq f_{p} \leq \delta\right\}\right)-\chi\left(V_{W}(G) \cap\left\{f_{p}=\delta\right\}\right)- \\
\chi\left(V_{W}(G) \cap\left\{f_{p}=-\delta\right\}\right) .
\end{gathered}
$$

Dos itens $(a)$ e $(b)$ acima, segue que os dois primeiros termos do lado direito desta igualdade são iguais a $\chi\left(M_{F}\right)$. O terceiro termo é igual a $\chi\left(V_{W}(F)\right)$, pois Durfee [Dur] mostrou que $V_{W}(F)$ é um retrato por deformação de $V_{W}(G) \cap\left\{-\delta \leq f_{p} \leq \delta\right\}$ e os dois últimos termos são os bordos $M_{F}^{\delta}$ e de $M_{F}^{-\delta}$. Como $V_{W}(G) \cap\left\{f_{p}=\delta\right\}$ e $V_{W}(G) \cap\left\{f_{p}=-\delta\right\}$ são os bordos das variedades $M_{F}^{\delta}$ e de $M_{F}^{-\delta}$, respectivamente. Temos que as duas últimas características de Euler são iguais a 0 , se $n-p$ é par e iguais a $2 \chi\left(M_{F}\right)$, se $n-p$ é ímpar. O que completa a prova da Proposição.

Vamos escolher $l \in\{2, \cdots, p\}$ e uma $l$-tupla $I=\left\{i_{1}, \cdots, i_{l}\right\}$ de pares de elementos distintos de $\{1, \cdots, p\}$. Seja $J=\left\{i_{1}, \cdots, i_{l-1}\right\}$. Se $l=1$, então, façamos $J=\emptyset$ e $f_{J}=0$. Observe que neste caso $V_{W}\left(f_{J}\right)=W$. Assim, temos o resultado mais geral como segue.

Proposição 5.14 Temos $\chi\left(V_{W}\left(f_{J}\right)\right)-\chi\left(V_{W}\left(f_{I}\right)\right)=(-1)^{n-l} 2 \chi\left(M_{F}\right)$.

Demonstração: Mesma prova da Proposição 5.13.

Em particular, obtemos os dois Corolários a seguir.

Corolário 5.15 Seja $j \in\{1, \cdots, p\}$. Então, $\chi\left(V_{W}\left(f_{j}\right)\right)=\chi(W)-(-1)^{n-1} 2 \chi\left(M_{F}\right)$.

DemonstraÇÃo: $\quad$ Basta aplicar a Proposição 5.14 para o caso $l=1$. 
Corolário 5.16 Sejam $l \in\{3, \cdots, p\}$ e $I=\left\{i_{1}, \cdots, i_{l}\right\} \subset\{1, \cdots, p\}$. Considere $K \subset$ $I$ uma lista qualquer de $(l-2)$ inteiros distintos de $I$. Então, temos que $\chi\left(V_{W}\left(f_{K}\right)\right)=$ $\chi\left(V_{W}\left(f_{I}\right)\right)$.

Demonstração: Seja $J$ uma lista com $(l-1)$ elementos distintos obtida de $K$ adicionandose um elemento de $I \backslash K$. Pela Proposição 5.14, o Corolário segue da igualdade

$$
\chi\left(V_{W}\left(f_{J}\right)\right)-\chi\left(V_{W}\left(f_{I}\right)\right)=\chi\left(V_{W}\left(f_{J}\right)\right)-\chi\left(V_{W}\left(f_{K}\right)\right)
$$

Por fim, para expressarmos a característica de Euler de todos os conjuntos $V_{W}\left(f_{I}\right)$ é suficiente calcular a característica de Euler de um conjunto $V_{W}\left(f_{I}\right)$ quando $\# I=2$. Mas, para o conjunto $I=\{1,2\}$, segue da Proposição 5.14, que

$$
\chi\left(V_{W}\left(f_{I}\right)\right)=\chi\left(V_{W}\left(f_{1}\right)\right)-(-1)^{n} 2 \chi\left(M_{F}\right) .
$$

Portanto, podemos resumir todos estes resultados no seguinte Teorema.

Teorema 5.17 Sejam $l \in\{1, \cdots, p\}$ e $I=\left\{i_{1}, \cdots, i_{l}\right\}$ como acima.

(a) Se n é par, então $\chi\left(V_{W}\left(f_{I}\right)\right)=\left\{\begin{array}{l}2 \chi\left(M_{F}\right), \text { se l é ímpar } \\ 0, \text { se l é par }\end{array}\right.$

(b) Se n é impar, então $\chi\left(V_{W}\left(f_{I}\right)\right)=\left\{\begin{array}{l}\chi(W)-2 \chi\left(M_{F}\right), \text { se l é ímpar } \\ \chi(W), \text { sel é par }\end{array}\right.$ 



\section{Referências Bibliográficas}

[A-C1] H. Aguilar-Cabrera, The topology of real suspension singularities of type $f \bar{g}+z^{n}$, Math. Z., 277, (2014), no. 1-2, 209-240.

[A-C2] H. Aguilar-Cabrera, New open-book decompositions in singularity theory, Geometria Dedicata, 158, (2012), 87-108.

[ACT] R. N. Araújo dos Santos, Y. Chen e M. Tibăr, Singular open book structures from real mappings, Central European Journal of Mathematics, 11, (2013), no. 5, 817-828.

[ACT1] R. N. Araújo dos Santos, Y. Chen e M. Tibăr, Real Polynomial Maps and Singular Open Books at Infinity, submetido para publicação (veja www.arxiv.org/abs/1401.8286v1).

[AD] R. N. Araújo dos Santos e N. Dutertre, Topology of real Milnor fibrations for non-isolated singularities, submetido para publicação (veja arxiv em www.arxiv.org/abs/1211.6233).

[ADD] R. N. Araújo dos Santos, D. Drebelbis e N. Dutertre, Topology of the real Milnor fiber for isolated singularities, Contemporary Mathematics, American Mathematical Society, 569, (2012), 67-75.

[Ar] R. N. Araújo dos Santos, Fibração de Milnor de Singularidades analíticas reais, Tese de doutorado, Universidade de São Paulo/Brasil, (2002).

[AT] R. N. Araújo dos Santos e M. Tibăr, Real map germs and higher open book structures, Geometria Dedicata, 147, (2010), 177-185.

[BCR] J. Bochnak, M. Coste e M. F. Roy, Real Algebraic Geometry, 36, Springer Verlag, (1998).

[BLM] E. A. Bartolo, I. Luengo e A. Melle-Hernández, Milnor number at infinity, topology and Newton boundary of a polynomial function, Math. Z., 233, (2000), no. 4, 679-696.

[Bo] A. Bodin, Milnor Fibration and Fibred Links at Infinity, IMRN International Mathematics Research Notices, (1999), no. 11, 615-621.

[BP1] A. Bodin, A. Pichon e J. Seade, Milnor fibrations of meromorphic functions, J. Lond. Math. Soc., (2) 80, (2009), no. 2, 311-325.

[BP2] A. Bodin e A. Pichon, Meromorphic functions, bifurcation sets and fibred links, Math. Res. Lett., 14, (2007), no. 3, 413-422. 
[BR] R. Benedetti e J. Risler, Real algebraic and semi-algebraic sets, 27, (1990).

[Br1] S. A. Broughton, Milnor numbers and the topology of polynomial hypersurfaces, Invent. Math., 92, (1988), no. 2, 217-241.

[Br2] S. A. Broughton, On the topology of polynomial hypersurfaces, Singularities, Part 1, Arcata, Calif., (1981), 165-178.

[Ch] Y. Chen, Bifurcation values of mixed polynomials and Newton polyhedra, Tese de doutorado, Universidade de Lille 1/França, (2012).

[CS] M. J. D. Carneiro e M. G. Soares, Introdução à topologia de singularidades complexas, Instituto de Mathemática Pura e Aplicada, (1985).

[DAYA] N. Dutertre, R. N. Araújo dos Santos, Y. Chen e A. A. do Espirito Santo Open Book Structures on Semi-algebraic manifolds, submetido para publicação (veja www.arxiv.org/abs/1409.4316).

[Di] L. R. Dias, Regularity at infinity and global fibrations of real algebraic maps, Tese de doutorado, Universidade de São Paulo/Brasil, (2012).

[Dur] A. Durfee, Neighborhoods of algebraic sets, Trans. American Mathematical Society, 276, (1983), no. 2, 517-530.

[Er] C. Erhresmann, Sur les spaces fibrés différentiables, Compt. Rend. Acad. Sci. Paris, 224, (1947), 1611-1612.

[HL] H. A. Hamm e L. D. Tráng, Un théorème de Zariski du type de Lefschetz, Ann. Sci. Ecol. Norm. Sup, (4) 6, (1973), 317-355.

[Ja] A. Jacquemard, On the fiber of the compound of a real analytic function by a projection, Boll. Unione Mat. Ital. Sez B, Artic. Ric. Mat., (8) 2, (1999), no. 2, 263-278.

[JJJ] J. Cisneros, J. Seade e J. Snoussi, Milnor fibrations and d-regularity for real analytic singularities, International Journal of Mathematics, 21, (2010), no. 4, 419-434.

[Kh] G.M. Khimshiashvili, On the local degree of a smooth map, Soobshch. Akad. Nauk Gruz. SSR, 85, (1977), 309-311.

[KOS] K. Kurdyka, P. Orro e S. Simon, Semialgebraic Sard theorem for generalized critical values, J. Differential Geometry, 56, (2000), no. 1, 67-92.

[Lo] E. Looijenga, A note on polynomial isolated singularities, Indag. Math., 33, (1971), 418-421.

[Ma1] D. Massey, Real Analytic Milnor Fibrations and a Strong Eojasiewicz Inequality, Real and complex singularities, London Math. Soc. Lecture Note Ser., 380, Cambridge Univ. Press, Cambridge, (2010), 268-292.

[Ma2] D. Massey, Real and Complex Singularities, London Math. Soc. Lecture Note Series, 380, (2010), 268-292.

[Mil1] J. Milnor, Singular points of complex hypersurfaces, Ann. of Math. Studies, 61, Princeton University Press, (1968). 
[Mil2] J. Milnor, Morse Theory, Ann. of Math. Studies, 51, Princeton University Press, (1963).

[NZ1] A. Némethi e A. Zaharia, Milnor fibration at infinity, Indag. Math., 3, (1992), no. 3, 323-335.

[NZ2] A. Némethi e A. Zaharia, On the bifurcation set of a polynomial function and newton boundary, Publ. Res. Inst. Math. Sci., 26, (1990), no. 4, 681-689 .

[Ok] M. Oka Non-degenerate mixed functions Kodai Math. J., 33, (2010), no. 1, 1-62.

[Pa] A. Parusiñski, On the bifurcation set of a complex polynomial with isolated singularities at infinity, Compositio Math., 97, (1995), no. 3, 369-384.

[PZ] L. Păunescu e A. Zaharia, On the Eojasiewicz exponent at infinity for polynomial functions, Kodai Mathematical Journal, 20, (1997), no. 3, 269-274.

[RSV] M. A. S. Ruas, J. Seade e Verjovsky, On Real Singularities with a Milnor Fibration, Trends in Mathematics: trends in Singularities, Birkhauser Verlag Basel/Switzerland, (2002), 191-213.

[Th] R. Thom, Ensembles et morphismes stratifiés, Bull. of the American Mathematical Society, 75, (1969), 240-284.

[Ti] M. Tibăr, Polynomials and vanishing cycles, Cambridge Tracts in Mathematics, 170, Cambridge University Press, Cambridge, (2007).

[Wi] H.E. Winkelnkemper, Manifolds as open book, Bull. Amer. Math. Soc. 79, (1973), 45-51 
